\title{
Bio-inspired textures for functional applications
}

\author{
Ajay P. Malshe (1) ${ }^{\mathrm{a}, \mathrm{b}, *}$, Salil Bapat ${ }^{\mathrm{b}}$, Kamlakar P. Rajurkar (1) ${ }^{\mathrm{c}}$, Han Haitjema (2) \\ ${ }^{a}$ Department of Mechanical Engineering, University of Arkansas, Fayetteville, AR, USA \\ ${ }^{\mathrm{b}}$ Microelectronics-Photonics Graduate Program, University of Arkansas, Fayetteville, AR, USA \\ ${ }^{\mathrm{c}}$ Department of Mechanical Engineering, University of Nebraska, Lincoln, NE, USA \\ ${ }^{\mathrm{d}}$ KU Leuven, Mechanical Engineering Department, B-3001 Leuven, Belgium
}

\section{A R T I C L E I N F O}

\section{Keywords:}

Surface texture

Surface Modification

Bio-inspired

\begin{abstract}
A B S T R A C T
Textures are abundantly exploited in nature for securing superior functionalities including adhesion, color manipulation, anti-reflection, and drag management. Over millions of years, these advanced properties are endowed to various organisms on the planet to survive and adapt in harsh environmental conditions. Texture characteristics such as feature size, shape, periodicity, aspect ratio, orientation and hierarchy are critical in nature's 'tool-box'. Manufacturing of cutting-edge products require multifunctionalities for efficiency, durability and sustainability for improving the quality of life of growing population. This paper analyzes and discusses convergence and underlying science and engineering of well proven natural strategies of surface textures and their effective synthetic implementation in engineered products.
\end{abstract}

(C) 2018 Published by Elsevier Ltd on behalf of CIRP.

\section{Introduction: bio-inspired surface textures-nature's tool for delivering functional surfaces}

'It is not the strongest of the species that survives, nor the most intelligent, but the most responsive to change,' said Charles Darwin. Survival of the fittest is a famous phrase of Herbert Spencer, which describes the idea that, in nature, there is a competition to survive and reproduce. Survival and change are two critical terms, especially as species on earth over millions of years are in continuous combat with changing environments. These environments are typically harsh for organisms to live in, but despite these difficulties organisms have survived and thrived by building harmonic ecosystems within divergent environments including marshy and muddy; deep cold and snowy; hot, dry and desert; and more. Living organisms interact and interface with their surroundings through their outer layers, their surfaces which have adapted and evolved with novel but simple designs and functional properties in this process of combat and survival.

The surface (and sub-surface) of an organism, also called skin, is an important interface between an organism and its environment, serving a mission critical role in the process of adaptation and survival. Skin surfaces interact with aggressive environmental factors such as temperature, moisture, abrasive agents such as sand and ice, $\mathrm{pH}$, bacteria and viruses, light, mating abrasive or super smooth surfaces, etc. and their combinations. To combat these aggressive factors, skins have evolved unique design architectures that achieve multifunctional properties including

\footnotetext{
* Corresponding author.
}

(but not limited to) adhesion, hydrophobicity, hydrophilicity, thermal-management, anti-reflection, structural colors, and spherical vision as highlighted in Fig. 1.

The skins of organisms, from here onwards called surfaces, deliver these environment-specific properties by combining textures and material chemistries to exploit classical laws of physics to achieve superior functional properties. The term surface texture captures various facets of a construct including the repetitive arrangement of features, the various shapes and sizes of features (in 2-D and 3-D), and the hierarchical distribution of quasi-periodic connected structures in a multi-dimensional space

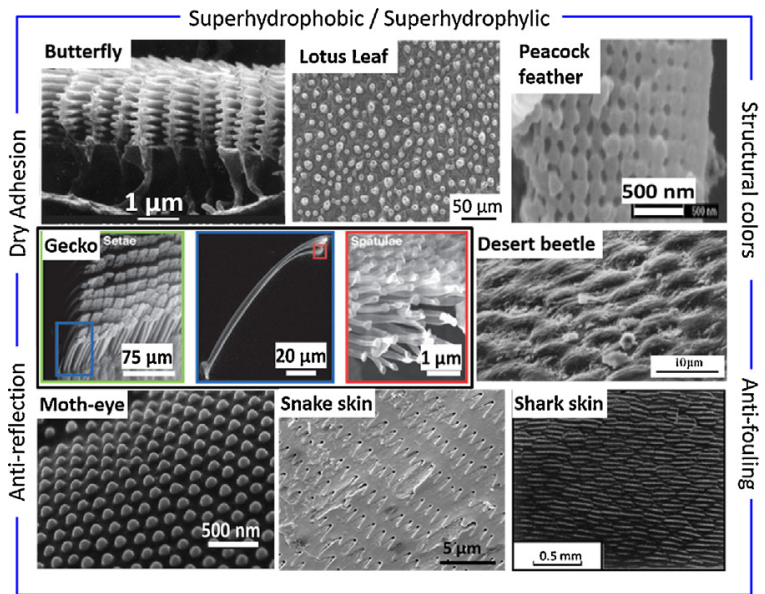

Fig. 1. Diversity in biological textures and their functions (images reproduced with permissions from $[20,26,61,100,157,180,247])$. 


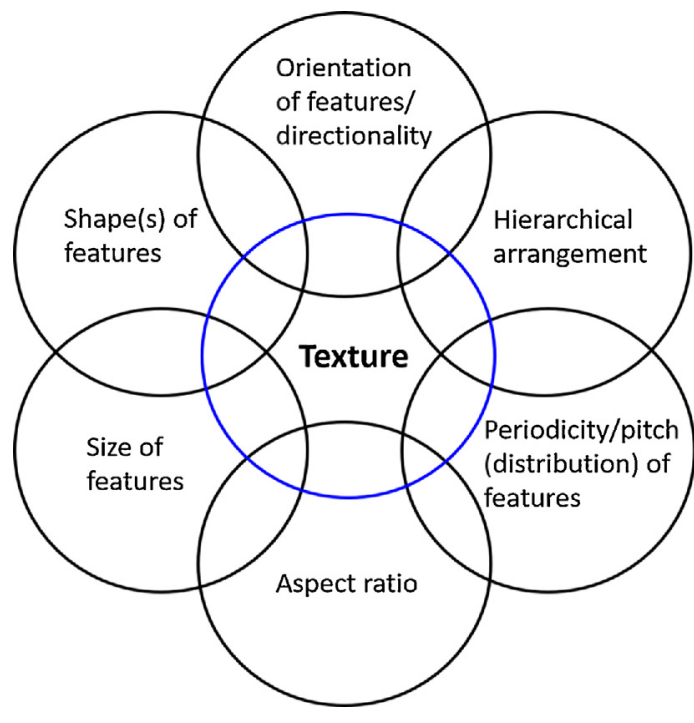

Fig. 2. Components that contribute to surface texture.

resulting in novel morphologies. Succinctly "surface texture is the geometrical irregularities present at a surface. Surface texture does not include those geometrical irregularities contributing to the form or shape of the surface" [168]. In other words, surface texture be seen as a design tool in nature's "tool box": they enable multifunctionality in many natural and biological surfaces [197]. Fig. 1 summarizes some of the commonly observed multi-functional textured surfaces in nature and the endowed functionalities of these surfaces.

To further understand texture as a design architecture or a construct, one needs to realize that all textures are convergence of construction features including the shapes of features, the sizes of features, their aspect ratio, their periodicity and pitch, their orientation and directionality, and their hierarchical arrangements (Fig. 2). As a result, there are more than 700 types of possible textures allowing a tremendous degree of freedom for surface manipulation. It is observed that these surface texture features converge to deliver a desired surface function (or multifunctions) to aid the organism's combat with the environment and survival.

Surface functions are also important for designing and manufacturing advanced products for growing populations. Furthermore, consumer demands are growing for higher efficiency and durability in products' performance and sustainability offered per unit currency of price. Also, demands are growing to deliver more functions per unit surface area per unit currency. Examples of such products are displays, personal data assistants (PDA), selfdriving cars, sustainable buildings, clothing, cosmetics, and more. Last but not least, with a growing global population, natural resources are dwindling, and well-established strategies are needed to engineer the next generation of sustainable products that can be produced by using less resources. Bio-inspired texture strategies have been refined over millions of years by organisms on earth and are examples of efficiency, durability and sustainability. These properties have made them a recent focus of products and production engineering.

Functional surfaces have been a subject of extensive scholarly research for decades $[78,91,161,173,230]$. Some of the most notable contributions through the years are the CIRP keynote papers in the areas of structured surfaces, engineering of functional surfaces, surfaces in precision engineering and bio-inspired functional surfaces $[44,64,77,197]$ along with notable contributions in surface metrology $[65,99,106,145,171,186,219,257]$. This paper builds upon these previous contributions and is unique in following discussion points on bio-inspired textures: (1) bio-inspired functional surfaces with an emphasis on specific design aspects of bioinspired "textures"; (2) methodical analysis of texture into construction components; (3) biological texture characteristics classified into Tier I and Tier II characteristics for designing surfaces for specific functionality; (4) effective convergence and implementation of these characteristics for realizing product applications where these textures are utilized.

In the following sections, this paper discusses specific examples of surface textures and their resultant functionalities in nature and the fundamental science that is utilized through these texture constructs to achieve these functions. This is followed by the elaboration on metrological approaches, process methodologies and instrumentations used for studying complex surface textures. Further, it is discussed and highlighted for product systems where such functions are of major values and the manufacturing processes used to realize advanced products by corporations.

\section{Understanding the science of surface textures in nature}

The following section describes the construct of surface texture and the underlying science along with the design strategies for manufacturing via examples of multi-functional textures observed in nature.

\subsection{Design components of surface texture}

Surface texture can be further analyzed into multiple design components as shown in Fig. 2. These components, along with the specific material properties, deliver the desired functionalities upon interaction with the environment. Based on the extensive analysis of the literature for multiple bio-inspired textures, it is realized that for specific surface texture enabled functions, certain texture design components dominate over the others. These primary (dominant) component(s), along with complementary secondary component(s), lead to the desired functional effect as illustrated by following examples. Over time, researchers have studied textured metals, semiconductors, polymers, ceramics and composites for delivering variety of functionalities and product applications. Examples of different materials are demonstrated later in this paper. Typically, selection of material(s) is guided by the intended function, application environment and manufacturing process capabilities.

\subsection{Natural examples of superhydrophobicity}

Lotus leaf is well known for its superhydrophobic and self-cleaning properties those are enabled by random and hierarchical arrangement of nodules and hairs at micro and sub-micron scales. Apart from lotus leaves, rice leaves also show superhydrophobic effects. However, in case of rice leaves, the surface features are arranged in a periodic manner along the length of the leaf, leading to directional and anisotropic wettability [79]. Several other plants' surfaces as well as some animal skins (such as water strider legs, peacock feather and butterfly wings) also exhibit multi-functional superhydrophobicity $[97,160,182,209,280]$. Rose petals are particularly interesting as they exhibit superhydrophobicity owing to convergence of hierarchical micro-scale and submicro scale features. However, the water droplets on rose petals have high adhesion to the surface so no roll-off effect can be observed $[25,180]$. Fig. 3 shows some of the natural superhydrophobic surfaces and their diverse texture features.

The wetting properties of a solid depend on the interfacial energy balance between the solid-liquid-air interfaces as depicted

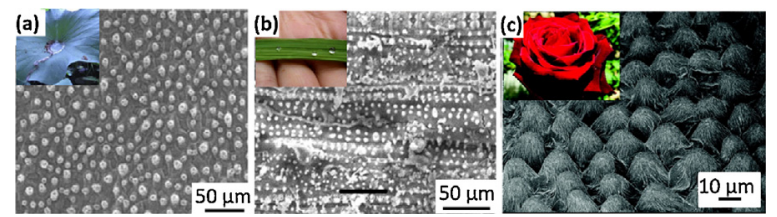

Fig. 3. (a) Surface structure of lotus leaf showing the micro-scale texture [180] (reprinted with permission from Elsevier); (b) photograph and SEM micrographs of rice leaf showing the directional micro-texture [96] (reprinted with permission from Elsevier); and (c) photograph and SEM images of rose flower and micro-texture [80] (reprinted with permission. Copyright 2008 American Chemical Society). 
(a) Young's equation
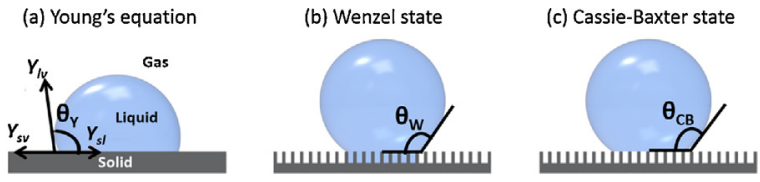

Fig. 4. Different wetting modes for a droplet on (a) flat substrate and (b)-(c) rough surfaces [240] (reproduced under the Creative Commons Attribution 4.0 Generic license).

by Young's equation (Fig. 4(a)) on a perfectly flat substrate. Wenzel [274] proposed an equation (Eq. (1)) that predicts the effect of surface roughness on contact angle. Where $r$ is the roughness ratio defined by the ratio of total surface area due to roughening of substrate over the original surface area before roughening and is always greater than 1 . Thus, the addition of surface roughness to flat hydrophobic surfaces increases their hydrophobicity [181]. When the right-hand side of Eq. (1) exceeds a value of 1 , liquid droplets transition from a Wenzel state (Fig. 4(b)) into a Cassie-Baxter regime (Fig. 4(c)) due to excess increase in solidliquid surface area and associated increase in interfacial energy. In a Cassie-Baxter regime, the liquid partially wets the surface leading to the formation of stable air pockets. The Cassie-Baxter model [50] is described by Eq. (2), where $f_{s}$ is the fraction of solidliquid interface. In the case of hierarchical roughness features, the wetting phenomenon is driven by multiple components and can have different wetting scenarios summarized in following report [28].

$\cos \theta_{W}=r \cos \theta_{Y}$

$\cos \theta_{C B}=f_{S}\left(r \cos \theta_{Y}+1\right)-1$

The hierarchical arrangement of texture at micro and submicron scales enables selectivity in wettability characteristics. In the case of lotus leaves, the water droplets do not penetrate into the micro and submicron scale features and thus roll-off with even the slightest tilt while collecting dirt particles and enabling efficient self-cleaning [26]. With the example of rice leaves, directional roll off is enabled by anisotropic orientation and arrangement of features. In the differing case of rose petals, water droplets are pinned due to penetration into the micro-scale valleys, leading to high contact angle hysteresis [28]. The transition between these various wetting states is governed by the size of the droplets as well as the submicron nanoscale feature sizes and their arrangement $[28,25]$. Thus, primarily, the hierarchical arrangement of features, size and their distribution along with surface chemistry are the primary components (Fig. 5) responsible for superhydrophobic effect.

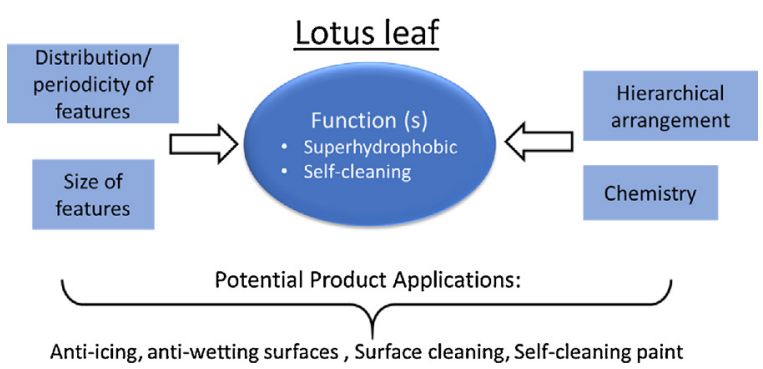

Fig. 5. Controlling design components for lotus-leaf inspired superhydrophobic/ self-cleaning surfaces and potential industrial product applications.

\subsection{Natural examples of dry adhesion}

Serval insects and animals such as beetles, flies, spiders and lizards [74] rely on their hairy terminal attachment structures to facilitate gripping on a variety of surfaces while walking. The adhesion mechanisms of geckos are of particular interest since geckos can walk easily on smooth substrates such as glass and also
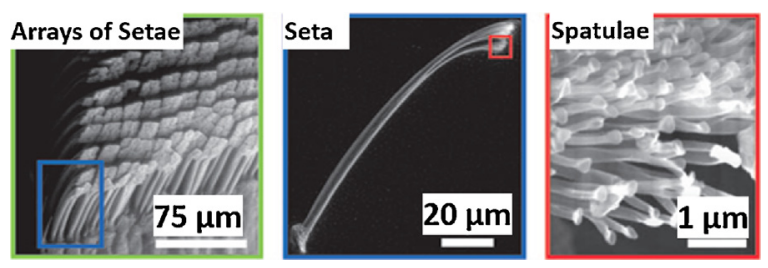

Fig. 6. Micrographs of gecko feet fibrillar structures showing the hierarchical arrangement and contact splitting [100] (copyright (2005) National Academy of Sciences, USA).

have the highest mass amongst species with fibrillar attachment pads. Fig. 6 shows the arrangement of setae on gecko feet and their branching [100]. The tips of each individual seta are further segmented into several spatula tips. The terminal shape of the features along with hierarchical arrangement is essential for the dry adhesion observed in geckos and other species [26]. Van der Waal's forces of attraction between spatula tips and substrate are shown to be the primary cause of adhesion for geckos [10]. This type of interfacial energy driven adhesion is differentiating from other excellent adhesion mechanisms found in nature that make use of a sticky glue as secreted by mussels (wet adhesion) or interlocking hook and loop structures as found in pollens and cocklebur seeds.

The adhesion force of a single tip is given by Johnson-KendellRoberts (JKR) theory [147] as shown in Eq. (3) below: adhesive pulloff force $\left(F_{C}\right)$ is related to the contact radius $(R)$ and adhesion energy per unit area $(\Upsilon)$. If the spatula tip is further subdivided into ' $n$ ' multiple identical smaller tips (known as contact splitting) while keeping the area constant, then the adhesion force increases by a factor of $\sqrt{n}$ as shown in Eq. (4) [9]. Thus, multiple contact points enabled by hierarchical arrangement of hair collectively contribute to high adhesion forces generated by geckos. Apart from the primary contribution of attractive contact forces, other effects such as capillary forces are also responsible for adhesion in geckos [29].

$F_{C}=\frac{3}{2} \pi \gamma R$

$F_{C}^{\prime}=\sqrt{n} F_{C}$

The attachment strategy in geckos is unique since it allows for efficient dry adhesion as well as easy detachment or peel off. The pull off force decreases with the orientation of the tips with respect to a surface. Geckos detach their feet by curling their toes in such a fashion that the spatula tips are easily detached from the substrate [29]. Thus, apart from the size and shape of contact features, the orientation of hair with respect to the plane of mating surface also plays a key role in the dry adhesion shown by geckos. For designing a gecko-inspired adhesive, the elastic modulus of the materials as well as the aspect ratio of features should also be taken into account [246]. Primary design components and applications of gecko inspired surfaces are presented in Fig. 7.

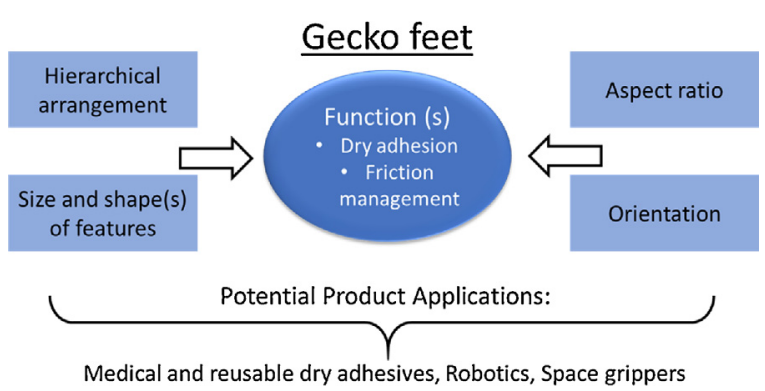

Fig. 7. Controlling components for gecko- inspired adhesive surfaces and potential industrial product applications. 


\subsection{Natural examples of antifouling and drag reduction}

Several marine species such as Mollusk shells and sharks have developed multifunctional surface structures that allow fast and efficient swimming under water and while resisting the adhesion of fouling species on their skin [31,32,233].

Shark skin surface features are widely studied for their antifouling as well as drag reducing properties. The surface structure of sharks consists of overlapping scales, known as dermal denticles arranged in a periodic manner. Fig. 8 shows the arrangement of dermal denticles in 2 species of sharks near the head region [20]. Microscopic details of each denticle (such as feature size, spacing, height) varies from one part of the body to the other, but the overall macroscopic arrangement of denticles remains similar [233]. Sharks orient the scales in the direction of their movement to align the ridges parallel to the flow of water, allowing swift movement underwater [32].

Apart from shark skin, microtopographies of several seashells also exhibit antifouling properties. Fig. 9 shows the typical ridge like micro-pattern from mytilid shells of species Mytilus edulis obtained from different parts of the world. It is evident from the figures that the spacing, arrangement and aspect ratio of the grooves is different for the same species in different environment [24].

The riblet structure of shark skin is believed to be essential for its drag reduction properties. The texture of the riblets enables shark skin to pin and lift the high-pressure vortices formed due to turbulent flow $[32,208]$. These vortices only contact the tip of the riblets. As a result, the overall contact area and shear stress are reduced, decreasing the drag experienced by sharks $[33,34]$. This phenomenon is illustrated in Fig. 10 and is similar to the drag reduction provided by dimples on a golf ball $[33,166]$.

Fouling is a time dependent dynamic process and hence is a complex phenomenon to model and understand. Several contributing theories have been proposed and are discussed below: (1) the formation of vortices and the efficient flow of a viscous boundary layer makes it difficult for fouling species to adhere on surface; (2) the continuous adjustment and re-orientation of riblets by sharks makes it harder for any micro-organisms to adhere on to a 'moving target' [26]; (3) 'attachment point theory' (illustrated in Fig. 11) suggests that fouling is reduced if the total number or attachment points (or the surface area) available for fouling species are reduced [231,234]. In reality, all of these mechanisms converge contributing

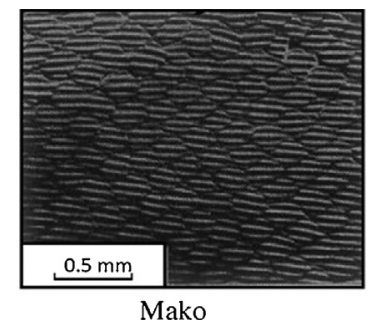

Isurus oxyrinchus

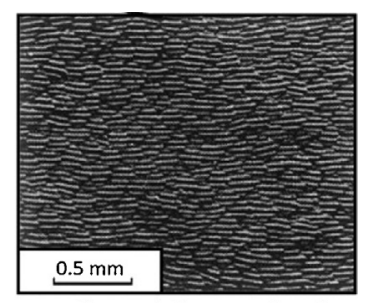

Smooth hammerhead Sphyrna zygena
Fig. 8. SEM micrographs showing scale patterns of two species of fast-swimming sharks [20] (reproduced with permission from Springer).

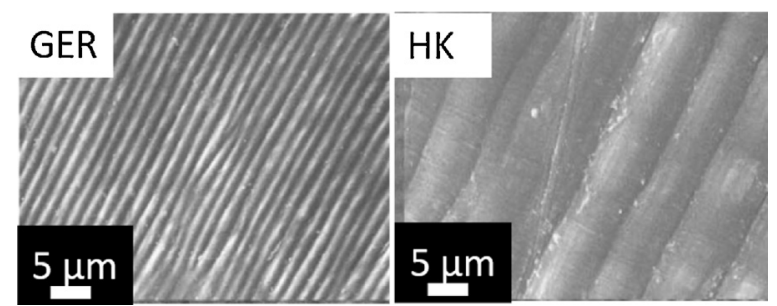

Fig. 9. SEM micrographs showing microtopographies of Mollusk species M. edulis from different geographic locations [24] (reprinted by permission of Taylor \& Francis Ltd.).
Shark skin effect: flow visualization with smoke from atomized oil (flow into the page)

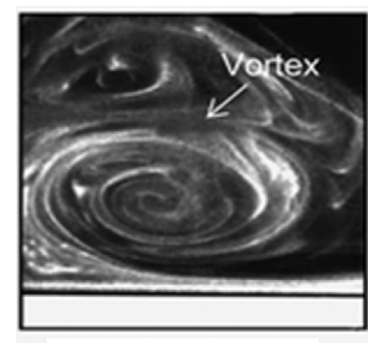

Flat: Drag $\uparrow$

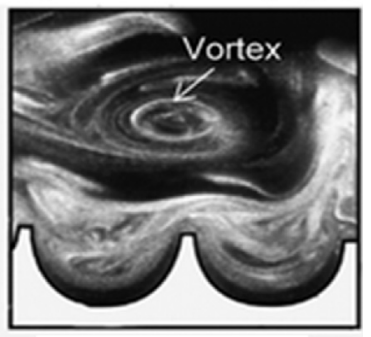

Riblets: Drag $\downarrow$
Fig. 10. Visualization of vortices generated during turbulent flow conditions on flat surface and riblet structure, width-200 $\mu \mathrm{m}$ [32] (reproduced with permission from The Royal Society of Chemistry).

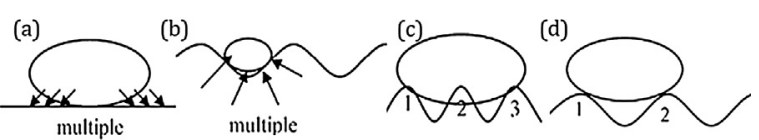

Fig. 11. Attachment point theory illustrating the effect of the relative size of features on the attachment of fouling species; strong adhesion and fouling is expected for (a) and (b) whereas weak adhesion and less fouling is expected in cases (c) and (d) [234] (reprinted by permission of Taylor \& Francis Ltd.).

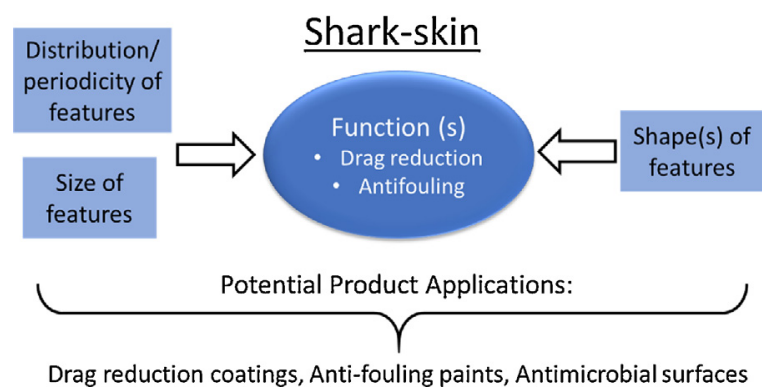

Fig. 12. Controlling design components for shark skin inspired anti-fouling/dragreducing textures and potential industrial product applications.

to the antifouling properties of shark skin to a certain extent and the complete science of the process is yet to be fully understood.

The effectiveness of a microtextured surface was evaluated using key surface texture components and show that size, periodicity and directionality of surface features play an important role in deterring species from attaching to a surface [232]. The above discussion illustrates that the size of the features, their arrangement, and the orientation of the features are the controlling components for the drag reduction and antifouling properties of the skin surfaces represented in Fig. 12. Further, it is important to note that material parameters such as surface chemistry as well as the external factors such as fluid flow, and the nature (size, shape, chemistry) of any fouling species will also have to be considered to design and fabricate bio-inspired artificial drag reducing and antifouling surface textures.

\subsection{Natural examples of tribology and locomotion}

Many reptile species such as snakes have evolved to have ordered scales on their ventral (belly region) surface that aid effective locomotion [2]. The scales on snakes are different in shape and size depending on the location they are found on the body [1]. Typically, ventral scales on the main body of a snake are hexagonal in shape, a common feature shape in many natural systems which allows the most efficient packing on in a repetitious fashion [3]. These scales are made up of micro-fibrils and denticulations arranged in a periodic manner $[4,3,19,103]$. The 


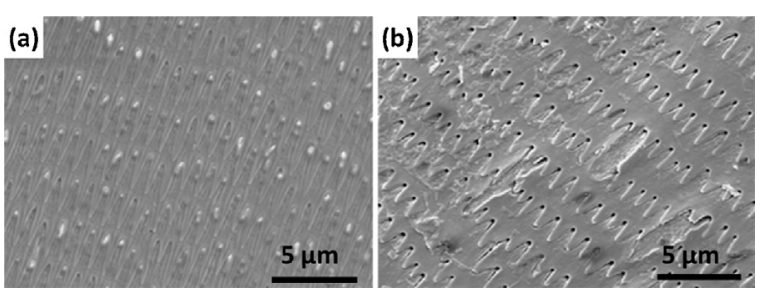

Fig. 13. SEM images depicting micro-structural details of the ventral scales of snake species, (a) Milk snake (L. triangulum), (b) King Cobra (Ophiophagus hannah) [61] () IOP Publishing. Reproduced with permission. All rights reserved).

microscopic fibrils are oriented in a caudal direction (towards the tail of the snake) and form a wavy pattern as depicted in Fig. 13 below [18,103]. The shape, aspect ratio, spacing and overall distribution of the microfibrils are specific to particular families of snakes and are a possible result of evolutions to aid survival in their respective habitat [4].

The frictional response and wear of interacting surfaces is a highly stochastic and complex behavior that is dependent on several environmental factors. Surface chemistry, roughness and mechanical properties as well as external factors such as load, speed, actual contact area between the two interacting surfaces and the presence of third body particles and lubricating fluids dictate the actual tribological response. Thus, even for snake skin and other natural tribological systems, the effect of surface features is very situational and environmentally dependent. Surface texture at the sub-micron level on snake skin controls the points of contact leading to effective and energy efficient forward motion while maintaining the ability to grip on irregular surfaces [185,199]. The orientation and directionality of fibrils create 'friction anisotropy' such that friction is reduced in the forward direction while it is increased in backward direction of motion as represented in Fig. 14 [1,127]. This ability of snakes to actively control their scale orientation allows them to maneuver on different terrains and prevents slipping. Furthermore, the skin membrane also shows the presence of micropits and micropores that may act as delivery mechanisms or reservoirs for lubricating fluids $[2,4,103]$.

As noted in the reported study, the interlocking between the scales on a snake's skin and a mating surface roughness is essential for effective locomotion [81]. Unidirectionally orientated microscales and submicron sized denticulations are responsible for friction anisotropy [81]. Similarly, study of jumping insects such as planthoppers revealed a microscopic interlocked ratcheting gear system between the two feet (Fig. 15) of the insect [45]. These gears, textured on the surface, engage at the time of jumping and aid the synchronous unidirectional movement of the legs and an effective launch by the insect [45].
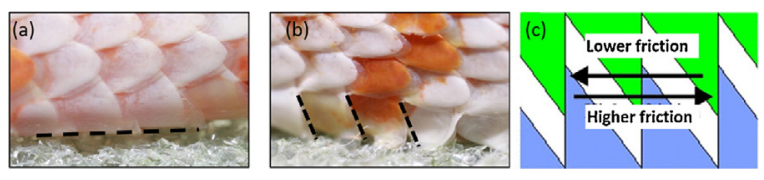

Fig. 14. Active control of snake skin scales showing change in orientation of scales on styrofoam substrate (a-b) [199] (reproduced with the permission of The Royal Society); and (c) schematic mechanism of friction anisotropy based on interlocking [185] (reprinted with permission from Elsevier).

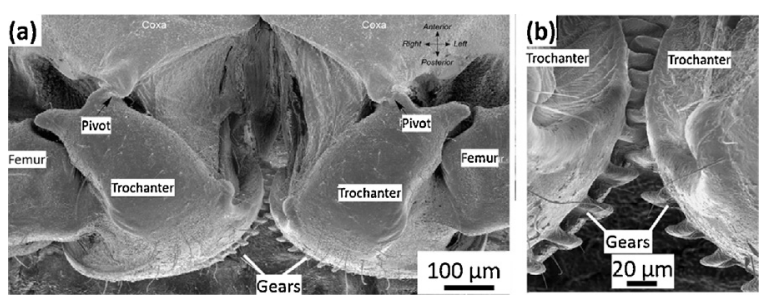

Fig. 15. Gears on the trochanter of grasshopper of species Issus nymph showing the effective interlocking [45] (reprinted with permission from AAAS).

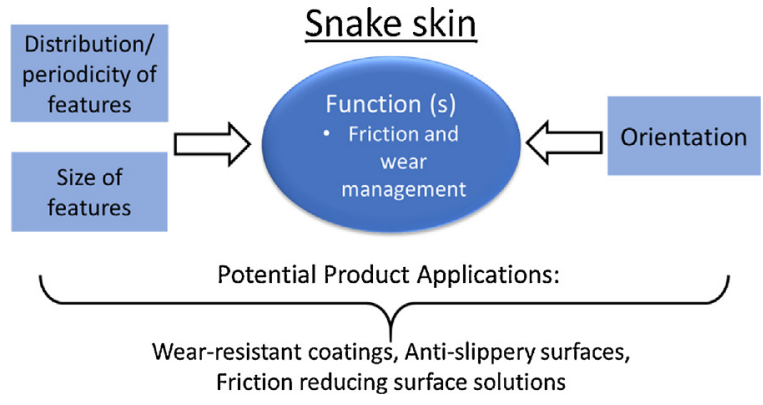

Fig. 16. Controlling design components for snake-skin inspired textures and potential industrial product applications.

From these observations it is found that the shape, orientation (actively controlled), size (necessary for effective interlocking depending on the surface roughness of the surface) and arrangement/periodicity of microscopic features are the controlling components for delivering effective friction and wear control as shown in Fig. 16. These components will be useful to design artificially textured tribological surfaces with tailored friction and wear response.

\subsection{Natural examples of anti-reflective surfaces}

Moth-eye structures have been an area of interest due to their excellent broadband anti-reflective (AR) properties and the related product applications $[43,47,179]$. The antireflective properties of moth eyes are due to a periodic arrangement of sub wavelength structures (SWS) on their facet lenses [224]. Similar features that lead to anti-reflective functionality were observed on various species of butterflies as well as cicada and termite wings $[23,22,70,204,215,243,252,271]$. The nano-nipples on various species show a regular periodic arrangement primarily in a hexagonal array as seen in Fig. 17. However, the height, shape and periodicity of these submicron scale features differ from one species to the other. This can be attributed to the evolution of species to serve a desired function in a specific environment and are interpreted as key texture components for anti-reflective surfaces.

Typically, in order to achieve efficient anti-reflection, the height of the individual features needs to be of the order of half of the wavelength of incident light. Furthermore, the spacing between the features is also of the same order, which ensures that the array is not resolved by the incident light [224,277]. This causes the light to interact with the structure in such a way that the average refractive index (RI) at any given height ' $h$ ' is governed by the air/material volume ratio [179]. The effective RI at any given height $\mathrm{h}\left(n_{\text {eff }}(h)\right)$ can be calculated by using Eq. (5) where $n_{\mathrm{m}}$ is the RI of material, $A_{n}(h)$ is the cross-sectional material area at height $h$ and $A_{b}$ is the base area of sub-wavelength feature as shown in Fig. 18(a). Thus, the refractive index of the surface layer changes gradually from the top to the bottom along the height of the nipples as depicted in Fig. 18(b). This gradual change of RI results in the progressive bending of light at each layer, leading to anti-reflective behavior [47,277]. This strategy is different from multi-layer antireflective coatings that rely on destructive interference of reflected light at each layer interface [47]. Such arrays show good anti-reflective behavior that is

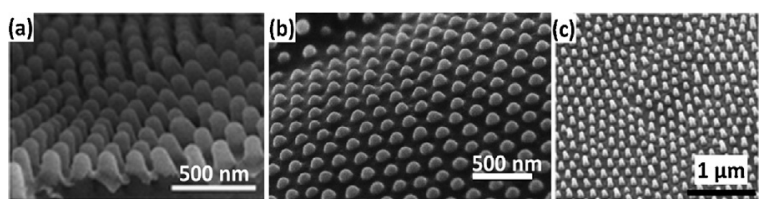

Fig. 17. SEM micrographs of nano-nipples in (a) Attacus atlas moth eye [159] (reproduced with permission of The Royal Society of Chemistry); (b) lycaenid butterfly Pseudozizeeria maha [247] (reproduced with the permission of The Royal Society); (c) cicada wing of Meimuna durga [252] ( IOP Publishing. Reproduced with permission. All rights reserved). 

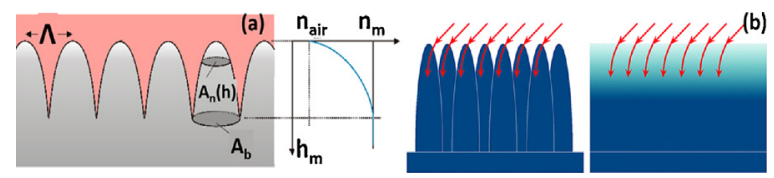

Fig. 18. Schematic showing the gradual change of the refractive index in subwavelength moth-eye nanostructures. Incident light, independent of the angle of incidence sees the nanostructure array as a gradually changing refractive index medium and is transmitted with reduced reflectivity $[47,205]$ ((a) reproduced under the Creative Commons Attribution 4.0 Generic license and (b) reproduced with permission of The Royal Society of Chemistry).

broadband and omnidirectional since the light sees the subwavelength structures (SWS) as a medium with a gradually changing RI irrespective of the incident angle. This aspect makes moth-eye structured coatings suitable for many product applications. A similar approach is implemented in designing efficient anti-echo walls and microwave enclosures [277].

$n_{e f f}(h)=\frac{n_{m} \times A_{n}(h)}{A_{b}}+\frac{A_{b}-A_{n}(h)}{A_{b}}$

It has been shown through observation and simulation studies that the paraboloid shape of the nipples is critical and most efficient for effective AR while the height of the nipples controls the bandwidth of the wavelengths that can be effectively trapped $[22,69,247,252]$. Thus, the aspect ratio of the features (height), the shape of the features and the periodicity are the controlling components for designing efficient AR structures for a given range of wavelengths as represented in Fig. 19.

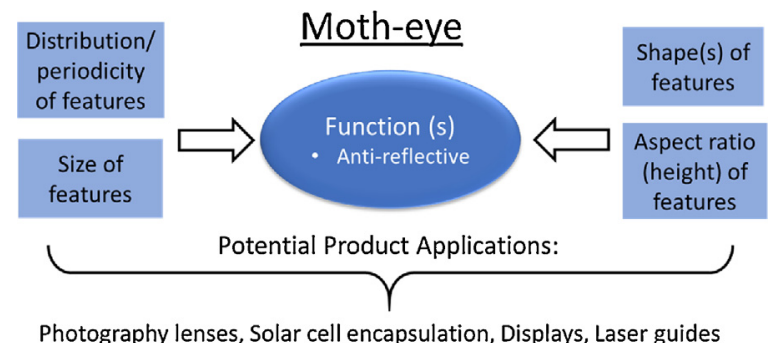

Fig. 19. Controlling design components for moth-eye antireflective textures and potential industrial product applications.

\subsection{Natural examples of photonic structures and iridescence}

Butterfly wings are an excellent example of multi-functional surfaces that show a diverse range of surface textures. Structural coloration or iridescence is one of the widely studied functional attributes of butterfly wings [133,180,197,210,280]. Structural colors are a result of selective reflection (and trapping) of specific wavelength(s) either due to optical interference/diffraction effects or due to the formation of a photonic band gap $[85,250]$.

Depending on the alternative and periodic arrangement of features with different RI, these structures can be classified in 1-D (periodic in 1 direction) to $3-\mathrm{D}$ (periodicity in all 3 directions) photonic structures [265,282]. 1-D gratings can be found on surface of plants such as Tulipa and Hibiscus [275], Mollusk shells Pinctada margaritifera, [184] and blue morpho butterflies [157] (texture/periodicity in cross sectional arrangement). Peacock feathers and butterfly Papilio nireus [189] are examples of 2-D structures while 3-D photonic structures, observed in gemstones such as opals, are also observed in beetles and the Parides sesostris butterfly [184,265,298]. Fig. 20 depicts representative examples of 1-D, 2-D and 3-D structures observed in nature. Apart from butterflies, several other species such as beetles [237], fish [86] and plants [40] also show structural colors. The attractive colors in natural systems serve a variety of functions including enticing prey, camouflaging for survival, attracting mates, or transferring pollen $[250,265]$. Structural color is a result of the interaction of

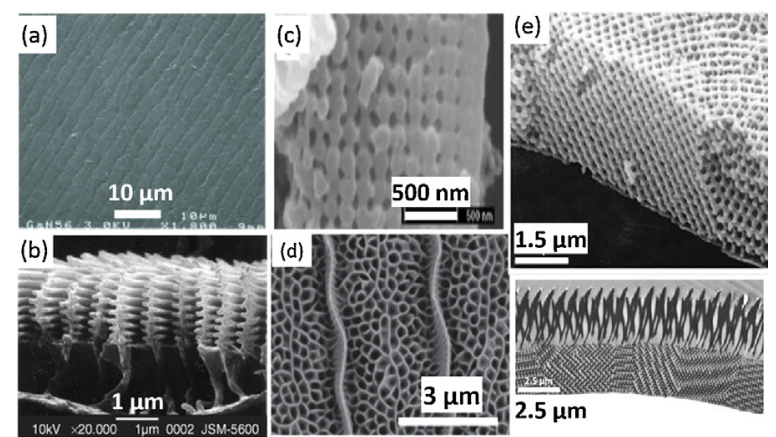

Fig. 20. Examples of the variety of iridescent photonic structures in nature: 1-D structures of (a) grooves on the shell surface of Mollusk Pinctada margaritifera [184] (@1999 Optical Society of America) (b) cross-section of ground scales of M. didius [157] (reproduced with permission from John Wiley And Sons); 2-D structures of (c) peacock feather and transverse SEM image of barbule [180] (reprinted with permission from Elsevier) (d) top view of cover scales of butterfly Papilio nireus [189] (reproduced with permission of The Royal Society of Chemistry); and 3-D structures of (e) inverse opal structures appearing in the green color of parides sesostris [264] (reprinted by permission from Nature Publishing Group).

incident light with surface and subsurface physical as well as chemical textures; the mechanisms of reflective color formations are discussed below.

Color exhibited by Mollusk shells (Fig. 20(a)) can be explained by simple diffraction grating. The incident white light is diffracted from the surface features and decomposed into different wavelengths. The scattering angle for each wavelength is governed by the order of diffraction $(\mathrm{m})$ and spacing between the grooves (d) as represented by Eq. (6). The resultant color is dependent on the incident angle and viewing angle as well as the order of diffraction [216].

$\mathrm{d}\left(\sin \theta_{i}-\sin \theta_{m}\right)=m \lambda$

Multi-layer interference is the primary reason for blue color exhibited by morpho butterflies. Incident light rays travel through alternate multi-layer stacks of chitin (high RI material) and air (texture in Z-direction). Light rays are then subsequently reflected, refracted and transmitted at each layer boundary [133]. In a morpho butterfly, the thickness (in Z-direction) of each layer is about $250 \mathrm{~nm}$ (Fig. 20(b)) which leads to preferential reflection of blue color due to constructive interference [85]. While reflection of other wavelengths is suppressed due to destructive interference. Additionally, the width of each individual shelf (about $300 \mathrm{~nm}$ ) is less than wavelength of visible light which leads to the blue color being diffracted to a wider angle [229]. Also, randomness in height of the individual shelves (caused by subsurface waviness) suppresses multi-color interference $[157,229]$. These design components of texture, together, lead to brilliant blue iridescence of morpho butterflies.

3-D photonic structures in nature are a robust form of structural color. Just as the interaction of electrons with periodic crystal lattice leads to the formation of electronic band structures in solids, the interaction of photons with a 3-D periodic arrangement of domains with different RI leads to the formation of photonic bands and band gaps [85]. In the absence of external sources, Maxwell's equation can be simplified as Eq. (7) where $\mathrm{H}(\mathrm{r})$ is the magnetic field of photon, $\omega$ is the frequency and $\varepsilon_{\mathrm{r}}$ is the macroscopic dielectric function of the material. For a given 3-D photonic structure, Eq. (8) can be solved with appropriate boundary conditions imposed by the arrangement of periodic domains and their RI values to give a complete photonic band diagram in 3-D. The resultant photonic band gap leads to the complete reflection of a specific wavelength irrespective of the incident angle [133]. Such 3-D photonic structures are also seen in gemstones such as opals.

$\nabla \times\left\{\frac{1}{\varepsilon(r)} \nabla \times H(r)\right\}=\frac{\omega^{2}}{c^{2}} H(r)$

For all the photonic structures described above, the size of the features (of the order of wavelength of light) along with the periodicity/arrangement of the features are the primary compo- 


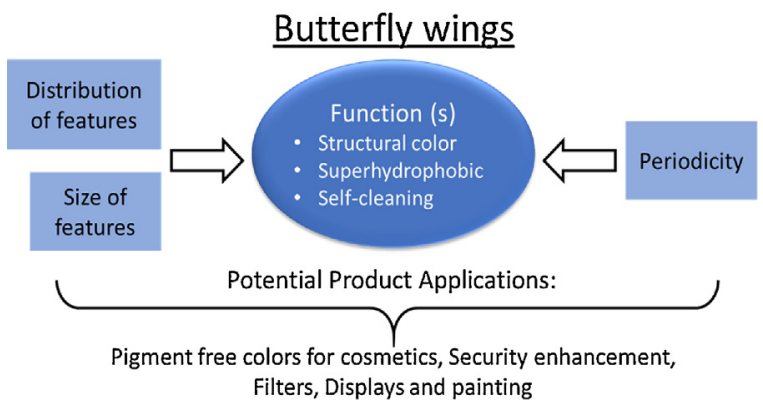

Fig. 21. Controlling design components for structural coloration and potential industrial product applications.

nents that contribute to the structural coloration as represented in Fig. 21. These controlling components, along with RI of material, are crucial in designing synthetic structural color structures. Furthermore, if the components such as spacing can be controlled via external stimulus such as the electric/magnetic field, temperature, or $\mathrm{pH}$ then the resultant colors can be tuned accordingly [229]. Such tunable structural colors are also seen in a variety of natural systems such as blue damselfish [86] and neon tetra-fish [85] and are very promising for numerous industrial product applications such as sensors, security enhancement and displays [282].

\subsection{Natural examples of chemical texturing}

Apart from the examples discussed above, certain plants and animals show unique functionalities enabled by a combination of the physical attributes of texture and the variation of surface chemistry in distinct domains. This subsection illustrates the use of surface chemistry as a functional design attribute with the help of the desert beetle Stenocara sp. and water floating fern Salvinia molesta as illustrative examples.

It has been reported that beetles from the Namib Desert use the surface structures on their backs to capture and harvest water from the moisture in the air [217]. Fig. 22(a) shows the surface structures of fog harvesting beetles. The back of the beetle is made up of microbumps and microchannels [182]. Furthermore, the morphology is also divided into superhydrophilic and superhydrophobic domains. The superhydrophilic tips are responsible for capturing moisture from the air [217]. Once the water droplet size grows above the critical size, it rolls off via the superhydrophobic channels directly into creature's mouth and thus is collected $[26,217]$. This beetle is unique in the way that it makes effective use of both physical texture as well as chemical texture. The spatial arrangement of alternate hydrophilic/hydrophobic domains combined with the physical channels for water movement enable the desert beetle to harvest water from the moisture in air, which is

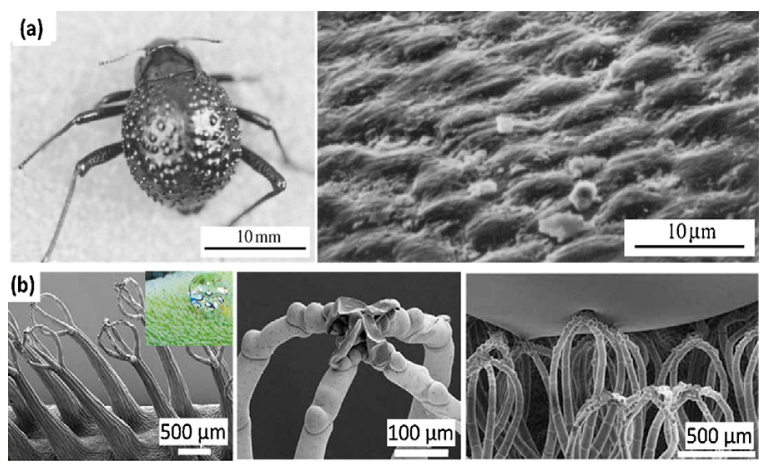

Fig. 22. (a) Photograph of Namib desert beetle Stenocara sp. and SEM micrograph showing bumps on its back used to capture moisture [26] (reprinted by permission from Nature Publishing Group); (b) water droplet on water fern Salvinia molesta (inset) and SEM images showing details of 'egg-beater' shaped hairs and their terminal cell morphology at different magnifications [15] (reproduced with permission from John Wiley And Sons). necessary for survival in the extreme conditions of the desert. Along with the Stenocara desert beetle shown in Fig. 22(a), other varieties of fog basking beetles have also been investigated [211]. It was found that the architecture on fog basking beetles has microridges and grooves, possibly physically aiding the water collection [211].

Thus, it can be concluded that the arrangement and distribution of features (along with their size) serve as the primary surface texture components for bio-inspired water harvesting surfaces. It should also be noted that the chemistry (hydrophilic and hydrophobic) serves as a material design parameter for synthetic water harvesting design architectures.

On the other hand, the hierarchical hairy architecture of the plant S. molesta along with the surface chemistry of its cuticular wax enables superhydrophobicity as depicted by a spherical droplet on the surface of the leaf (Fig. 22(b)-inset) [51,162]. The surface of these plants is superhydrophobic but not self-cleaning as the water droplet is retained on the surface and does not roll off $[162,163]$. Low temperature scanning electron microscopy on a frozen leaf revealed the unique design architecture of the hairs [15]. Fig. 22(b) depicts the egg-beater shaped terminal structures observed on each hair. Also shown is the terminal attachment point where four individual hairs attach and collapse to form a patch of dead cells. The entire leaf surface is covered with hydrophobic wax crystals except the terminal cells [15]. This arrangement of hydrophilic tips enables the effective pinning of the air-water interface and aids the formation of stable air pockets [131]. It was further noted that the air pockets formed on the Salvinia surface features are stable for several days [160] and are beneficial for reducing drag [8]. Similar to the desert beetle case, the size of the features and the distribution of chemical domains plays a critical role in the formation of stable air pockets and can be viewed as the design components for bio-inspired functional applications [8] as depicted in Fig. 23.

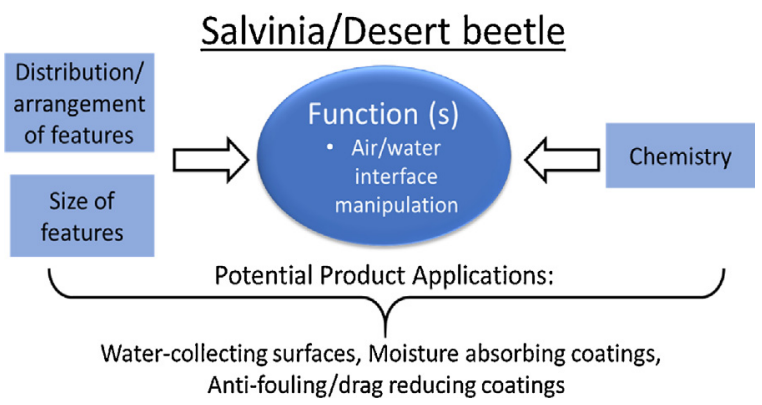

Fig. 23. Controlling design components for fog harvesting desert beetle surface and Salvinia along with possible product applications.

\subsection{Interaction of surface texture with external agents}

Further along with the above uniqueness in nature, there are additional characters, which make this topic intriguing. As a surface or a texture here is an interface between an organism and the surrounding environment, functionalities are result of the interaction between the design and material components of the texture and the environmental parameters. Natural surface textures have evolved in such a way that the system reacts to the external factor(s) to enable the desired functionality over short or extended period of times. During this interaction, the surface has dynamic responsive characteristics, for example the ability to refresh and/or regenerate to maintain the pristine character of the functionalities. Engineered systems and their synthetic textures, discussed below, are at the early stage of development.

Further, in addition to the design components discussed above, nature also demonstrates the following characteristics necessary for building a robust textured system: (1) redundancy for example in features; (2) simplicity of design and of choice of 
materials; (3) regeneration ability; (4) calling (multi-) functionality on-demand; (5) length scale dependent architectures (in addition to hierarchy discussed above); (6) heterogeneous integration of materials and (7) complementary subsurface with or without sensory system [197]. Furthermore, it appears that nature has built precision in systems' functional response at the macroscopic level like the magenta color in butterflies but has built defects at micron and submicron length scales. This can be illustrated by the imperfect arrangement of the lamellae on butterfly wing as depicted in Fig. 20(b). These defects, irregularities and randomness are crucial to provide the necessary robustness to the surface and build redundancy. A more detailed understanding of these characteristics will be important in order to design robust and adaptive textures that can be manufactured and utilized in a broad range of environments with wide functionalities.

The following section underlines the current approaches and areas of development for surface texture measurement. The measurement of the design components of novel and complex bioinspired surface textures discussed above is necessary to understand and replicate them in engineered systems.

\section{Measurement of surface texture and parameters according to ISO standards}

\subsection{General considerations}

In the bio-world, the 'image' of a surface is the most natural basis for further investigation. The human eye is trained and accustomed to 'looking' to - mainly - 2-D images and draws its conclusions even about 3-D features in a split second from recognition, relative sizes, shadow effects, contrast, etc. Most of the images in this field and also in this paper use this feature of human understanding implicitly. For example, insets in Fig. 3 omit a scale bar, which is not considered problematic as any human recognizes what is depicted. Images taken by scanning electron microscopy (SEM) especially benefit from this feature, as the imaging system gives shadow effects as they are observed by the human eye in real life [223]. One example - from many in this paper - is Fig. 17(b). This is essentially a 2-D image in grey tones, but it is readily interpreted as an array of small features on a surface, where the 'depth' is implied by gradual changes in the features towards the top of the image.

However, to interpret features in scientific concepts, i.e. being reproducible and having a predictive value, care must be taken when deriving quantitative measurement values and any related uncertainties.

The used measurement techniques must be appropriate for the length scale of the structures studied. For texturing, feature sizes typically range from the mm-size features that determine the tribological properties of shark skin, to the nm-size features of anti-reflective moth-eye structures.

Before any measurement task can be defined, the functional property under study must be defined: is it the anti-fouling, the color, the adhesive properties, etc. that is being studied? Following the design components that contribute to the surface texture as these are given in the scheme of Fig. 2, combined with the considerations that were discussed in Ref. [276], the following measurement tasks can be distinguished:

- Size of features-The relevant size can be a width that is defined by the distance between two opposing surfaces. Example: the width of grating lines in a Mollusk shell (Fig. 20 (a)). A feature size can also be defined as a height: the distance between two surfaces of same orientation but placed in a vertical direction. Example: the height of a moth-eye nanostructure (Fig. 17(a))

- Aspect ratio as defined by the depth of a structure divided by its width. Example: the aspect ratio of features of Gecko feet [246].
- The periodicity/pitch that can be defined as the average distance between features oriented in the same direction. Example: the grating distance on the Mollusk shell surface (Fig. 20(a)).

- The shape of features, e.g. the 'egg-beater' shaped hairs of a water fern (Fig. 22b)

- Profile and areal surface parameters defined as geometries of surface structures whose dimensions are small compared to the object under investigation. An example is the $R a$-value of snake skin as used in Ref. [1]. The full range and definitions of parameters are given in Refs. [134] and [136].

\subsection{Measurement traceability and calibration}

Measurement traceability is the property of a measurement result whereby the result can be related to a reference through a documented unbroken chain of calibrations, each contributing to the measurement uncertainty $[30,76,98]$. For measurements of biological textures, this implies that some calibration steps must be taken before traceability can be achieved. Calibration is multistep operation undertaken in specified conditions. In the first step, a relationship is established between the quantity values with measurement uncertainties provided by measurement standards and corresponding indications with associated measurement uncertainties. In the second step, this information is used to establish a relation for obtaining a measurement result from an indication $[30,48]$.

In the cases of measurement involving biological specimens, the calibration issue is often given little explicit attention as the nature of the measurement does not demand a reference standard for components as comparison. However, the aim of both calibration and traceability is that, in the end, an uncertainty can be attributed to a measurement, or to features of a bio-inspired product, which is relevant for designs based on these features.

\subsection{Commonly used instrumentation}

\subsubsection{Scanning electron microscopy (SEM)}

Topography images are usually obtained using SEM. SEM has some unique properties that, combined together, are not matched by any other microscopy technique. These include: magnification levels $(100 \times$ to $100.000 \times$ ), resolution down to $2 \mathrm{~nm}$ (for highest magnification), large depth of field, long working distance (allowing multiple positioning measurement strategies), elemental analysis capability and minimum diffraction effects [223]. However, SEM photographs are still inherently 2-D, and no height information can be extracted directly from the images. Furthermore, calibration of scales and uncertainty estimation is a prerequisite for the use of SEM pictures for quantitative evaluations. For example, the commonly used 'scale bar' in SEM micrographs can be considered to have a relative uncertainty between 1 and 5\% [14].

\subsubsection{3-D SEM}

To reconstruct the third dimension of surface features, photogrammetry methods can be used. 3-D information can be generated via reconstruction from stereo pairs [38]. Few results of such 3-D imaging with SEM are reported with a focus on the establishment of traceability [14]; it was shown that especially the height ( $\mathrm{z}$-) coordinates can have quite disappointing deviations up to $20 \%$. In an alternative approach [16], 3-D images of beetle skins are reconstructed by milling away $30 \mathrm{~nm}$ layers of the skin and taking a new SEM image after each step.

Most measurements and conclusions in the literature on bioinspired surfaces (especially where it comes to distances, heights, aspect ratio's and others) are based on SEM technology.

\subsubsection{3-D optical microscopy}

In optical microscopy, various methods can be used to obtain 3D information from an image. Several technologies are used for this 
type of microscopy: interference, confocal, and focus variation. However, each of these technologies have weaker and stronger points in vertical resolutions and carry the risk of measurement artifacts. Further details are given in handbooks [169]. Although they lack the extreme lateral resolutions of the SEM technology, the combination of the measurement of a relatively large area combined with good vertical resolution makes optical 3-D techniques most appropriate for the measurement of surface roughness parameters, though some issues must be addressed before these measurements can be considered as traceable and comparable [170,256].

\subsubsection{Scanning probe microscopy (SPM)}

SPM is a branch of microscopy that forms images of surfaces using a physical probe that scans the specimen. Commonly a feedback loop is used to maintain a constant force, or another physical phenomenon, between the specimen and the probe. In cases where the force is kept constant, the technique is called AFM (Atomic Force Microscopy). The resolution varies somewhat from technique to technique, but some probe techniques reach atomic resolution. The data are typically obtained as height coordinates on a two-dimensional grid of data points, making the data essentially 2.5-D, however for convenience the term 3-D will be used throughout this paper. 3-D AFM has the advantage of a better potential lateral resolution than optical techniques and compared to SEM technology it has the advantage of directly delivering 3-D coordinates. The horizontal and vertical ranges are well within the range of interest of bio-inspired surfaces: from a few $\mu \mathrm{m}^{2}$ to $1 \times 1 \mathrm{~mm} x-y$ range with a vertical $(\mathrm{z}-)$ resolution in the $\mathrm{nm}$ range and a range up to $10 \mu \mathrm{m}$. Although the measurement time can be rather long, the low measurement force combined with the appropriate range and resolution make this technology apropos for characterizing bio- and bio-inspired surfaces. These principles are discussed in [101], and more recently in several textbooks [60]. For larger scan lengths, direct contact mechanical probing can be used with a $\mu \mathrm{m}$ sized tip-radius. Because of the slow scanning speed these measurements are commonly restricted to 2-D [29] i.e. the height along a single line is measured.

\subsection{Profile and areal roughness parameters}

Surface roughness is characterized in parameters that are fully standardized for 2-D ('line') and 3-D ('surface') cases in ISO 4287 [136] and ISO 25178:2 [134], respectively. Apart from these definitions, the filtering conditions and probing conditions are essential, as given in ISO 4288 [137] and ISO 25178:3 [135]. These areal surface texture parameters that were standardized in 2012 were proposed and in use in the dimensional metrology community since the early 1990's [49].

The complex nature of bio-inspired surface implies that a combination of two or more parameters is desirable to make a useful distinction between surfaces. For example, the $S q$ parameter is sometimes called 'rms height'. It should be kept in mind that these parameters are all defined relative to a defined reference surface and depend on a defined bandwidth in terms of spatial frequencies. This bandwidth is too often neglected and present in the measurements in an implicit way, e.g. by the pixel resolution at the high-spatial frequency side and the size of the measurement area at the low spatial frequency side. The too-implicit treatment of the bandwidth concept seriously impedes the incredible potential these parameters have as general characterizing parameters for a wide variety of bio-inspired surfaces, from the mm-size features that determine the tribological properties of shark skin, to the nm-size features of moth-eye structures that have inspired the production of close-to-ideal optical AR structures.

\subsection{Examples of surface texture measurements}

In this section, specific examples of bio- and bio-inspired surface measurements are given for elaboration.

\subsubsection{Super hydrophobicity}

The dependence of super hydrophobicity on surface topography in general, with a particular focus on rose leaves was reported [28]. SPM measurements of $100 \times 100 \mu \mathrm{m}$ areas of rose leaves are taken, and own-defined features such as peak-to-base height, midwidth peak height, radius bump density, are determined from these measurements. As no uncertainty is estimated, it may be questioned whether a statement like "the mid-width is $16.7 \mu \mathrm{m}$ " is generally valid and can be reproduced by other researchers.

\subsubsection{Dry adhesion}

An original approach to solving the problem of time consuming 3-D macroscopic measurements was reported in context of studying the self-cleaning effects of Gecko feet [100]. The toe pad areas are 'measured' using a flatbed scanner, giving a quick image of the 2-D structures, while the finer structures at the $\mu \mathrm{m}$ level are measured using SEM.

\subsubsection{Anti-fouling}

A detailed study was performed on the anti-fouling properties of Mollusk surfaces [232]. Overall, 36 species were investigated using laser scanning confocal microscopy. The surface roughness parameters used in this study are mean roughness $\left(S_{a}\right)$, mean waviness $\left(W_{a}\right)$, skewness of the surface roughness profile $\left(S_{k}\right)$, skewness of the surface waviness profile $\left(W_{k}\right)$, texture aspect ratio $\left(S_{t r}\right)$ and fractal dimension $\left(F_{s k}\right)$. This study correlates these parameters to some measures of fouling resistance. The most significant correlation is found between anti-fouling properties and the skewness $\left(S_{k}\right)$ parameter. Still it is concluded that "the field is hindered by the lack of quantified surface characteristics to guide the development of new antifouling materials." This may be the case for several other properties of bio-inspired surfaces as well.

The same group put the measurement efforts at the $\mu \mathrm{m}$ and $\mathrm{nm}$ level in perspective by stating [233]: "While there are numerous new developments in surface modification technologies that tailor surfaces at the scale of nanometers, the over-riding theme for bioinspired materials is microstructured surfaces ranging from 1 to $300 \mu \mathrm{m}$. This focus is based on the extensive evidence from natural and manufactured surfaces that structures smaller than the settling propagule reduce settlement and attachment strength."

\subsubsection{Drag reduction}

Researchers [1], while studying snake skins, report use of 2-D roughness parameters from single lines that are extracted from 3D topography measurements taken by a white-light interferometer. In this case, a chance was missed to use all of the topography measured, to define 2-D parameters and to characterize the surface using these parameters.

In another paper [4], it was concluded, probably correctly, that the use of the $R_{a}$-parameter makes little sense and they specify parameters that would better characterize snake-skins (Fig. 24). However, a combination of spatial and areal parameters from ISO 25178:2 might have been more appropriate.

3-D measurements by laser confocal microscope and AFM to study snake surfaces were also reported [19]. Ra-values are discussed, but it is unclear how these were obtained, and it is likely

\begin{tabular}{ll}
\hline Parameter & LTS \\
TAR & $\frac{\text { Total area of dimples }}{\text { total surface area }}$ \\
$\mathrm{DSR}_{\mathrm{p}}=\frac{h_{\mathrm{p}}}{\Phi_{\mathrm{p}}}$ & $\begin{array}{c}\text { Total area of fibril tips } \\
\text { total surface area } \\
\mathrm{DSR}_{\mathrm{s}}=\frac{h_{\mathrm{s}}}{\Phi_{\mathrm{s}}} \approx \frac{R_{\mathrm{t}}}{\Phi_{\mathrm{s}}}\end{array}$ \\
$\mathrm{SAR}$ & $\mathrm{SAR}_{\mathrm{s}}=\frac{\lambda_{\mathrm{s}}+\Phi_{\mathrm{s}}}{h_{\mathrm{s}}}$
\end{tabular}

Fig. 24. Proposed Python-skin parameters as reported in Ref. [4] (@ IOP Publishing. Reproduced with permission. All rights reserved). 
that in fact the Sa-parameter values are used. The researchers [18] reported fabrication of snake-inspired micro structured polymer surfaces where the $R a$ - parameter is calculated from lines of a white light interferometry (WLI)-measurement. In this case as well, the Sa parameter could have been used, as the full 3-D topography was available.

\subsubsection{Anti-reflection}

A comprehensive study on moth-eye textures was published where the texture parameters measured on 20-different mothspecies using AFM combined with SEM measurements are reported [247]. This study even indicates measurement errors, mainly based on statistics. As the errors in the (mean) nipple heights are indicated as $10 \mathrm{~nm}$, the same scale used to round the nipple heights, the general validity of these values may be questioned, and the significance of the differences found.

Bio-inspired moth-eye and cicada wings structures are successfully applied as anti-reflection structures to solar cells. An example is given in Ref. [299], where measurement of bioinspired moth's eye nanostructures is reported. The produced surfaces are measured with AFM in tapping mode, and rms-values are reported that can be identified as the $S q$-parameter.

\subsection{Concluding remarks on surface texture measurements}

The development of advanced measurement instrumentation has enabled the measurement, modeling and reproduction of bioinspired surfaces with considerable success, but, the field would benefit from the consistent use of the defined ISO parameters on a more regular basis. On the other hand the ISO parameters are designed for surfaces produced by traditional mechanical engineering production technologies, and the field of bio-inspired surfaces could consider alternative evaluation methods such as the definition of surface texture parameters on freeform surfaces [212], or filtering free-form surfaces using the lifting wavelet transform [5]. Also the shearlet-based separation method of 3-D engineering surfaces [73] might open a way to quantify surfaces that are composed on an hierarchical arrangements such as the rose flower illustrated in Fig. 3. Also, the field would benefit from a better consideration of the concepts of bandwidth [170], traceability and uncertainty estimation [171] as that would make the measurements and conclusions more applicable and reproducible.

\section{Application specific bio-inspired functional engineered textures and their manufacturing}

The science of textures in the nature, discussed above, is being actively implemented in multiple real-world product applications. The following discussion elaborates on these applications, the related materials and manufacturing processes of choice and their implementation approaches.

\subsection{Bio-inspired textures for dry adhesion}

Fibrillar structures found in several lizards and insects especially geckos have attracted major research in last two decades as discussed in Section 2. The excellent ability of geckos to climb on a variety of substrates is remarkable for the following reasons: (1) the adhesive forces generated by geckos are primarily due to Van der Waals's forces of attraction and does not rely on use of any chemical glue; (2) the attachment is controllable, meaning that geckos can easily detach as well as adapt to different terrains by controlling the movement of the limbs and adjusting the orientation of attachment points. These features of dry adhesion are particularly interesting for several engineered applications such as robotic arms, grippers and others, which can be used in space and as biomedical adhesives. The following sections discuss the manufacturing methods and strategies as well as product applications for gecko-inspired surface textures.

\subsubsection{Manufacturing processes and approach}

As discussed in the previous section, the hierarchical arrangement, size and shape of terminal contact points, aspect ratio and orientation of features are important texture design components for fabricating fibrillar adhesive structures. A research group presented key design aspects for constructing efficient adhesive structures [246]. It is important to note that the aspect ratio, elasticity and stiffness of the fibrous structures are critical material and texture design attributes to consider for ensuring that the fibrous structures do not break during their intended target application. Ideal dry adhesive fibrillar structures should also have enough compliance to facilitate effective contact on variety of terrains and allow for possible 'switching' by controlling the fiber orientation [74,138]. Considering these design and material requirements, polymeric materials such as poly-dimethyl siloxane (PDMS) are most commonly used to develop synthetic bio-inspired dry adhesives.

The most commonly applied manufacturing approach involves top-down processing of the desired mold followed by the casting and demolding of textured polymers to fabricate gecko-inspired adhesive pillar structures (Fig. 25). The density of pillars can be controlled by changing the process variables used during the patterning process while the shape and size of terminal features can be controlled by adjusting the material removal process variables.

Fig. 26 shows examples of micropillar arrays with a variety of tip shapes and hierarchical arrangements. A master mold is first created using conventional photolithography and etching techniques. A soft curable polymer like PDMS or polyurethane (PU) can then be cast, cured and demolded to achieve desired array of pillars. Multiple photoresist patterning, developing and etching steps were used to create negative Si-master molds [146,294] and negative silicone rubber mold [151,152]. These molds can then be used to fabricate dual-shape/dual-level architectures using soft polymeric materials such as depicted in Fig. 26(a) and (b). In another approach, angled polyurethane acrylate (PUA) pillars (Fig. 26(c)) were first fabricated on a stiff polyethylene terephthalate (PET) backing layer using Si-master mold [270]. Two PET substrates with single sided pillars were then joined back-to-back

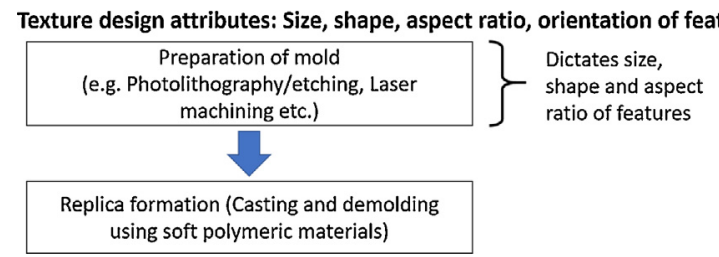

Fig. 25. Manufacturing approaches to fabricate dry adhesives.

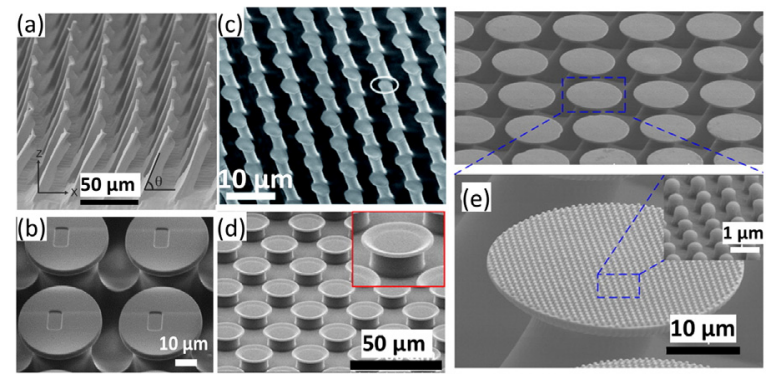

Fig. 26. SEM images of variety of structures fabricated by using lithographic techniques with different tip shapes, orientations and hierarchical arrangement: (a) polyurethane (PU) fibers with prismatic (first level) and rectangular adhesion pads (second level) [146] (reproduced with permission from John Wiley And Sons); (b) polystyrene micropillars with textured rectangular feature [151] (reprinted with permission. Copyright 2014 American Chemical Society); (c) dual angled polyurethane acrylate (PUA) pillars [270] (reproduced with permission of The Royal Society of Chemistry); (d) PDMS micropillars with mushroom shaped tips [286] (reprinted with permission, Copyright 2016 American Chemical Society); (e) Hierarchical PDMS mushroom shaped micropillars with nano cone tips [267] (reprinted with permission, Copyright 2014 American Chemical Society). 
to achieve double-sided adhesive structures. Separate micropatterned and nanopatterned masters have been used in the processing sequence to achieve dual-level hierarchical texture as seen in Fig. 26(e) [267]. Scalability of processes is key in their selection.

Apart from using conventional photolithography for patterning, several other processes for fabricating negative molds are also reported. A metallic mold with angled cavities was prepared via micro-end mill and used to fabricate anisotropic silicone rubber fibers with variety of tip shapes [238]. Similarly, holes created by milling [201] and CNC machining [132] were used to cast PDMS based micro and macro pillars. Laser cutting was used to manufacture PTFE molds containing arrays of holes in triangular, circular and square cross-sections those can be used to fabricate PDMS pillars [177]. A 3-D porous $\mathrm{Ni} / \mathrm{NiO}$ was used as a template in manufacturing. The template was prepared by electrodeposition of $\mathrm{Ni}$ from a nickel chloride and ammonium chloride electrolyte solution [292]. The hydrogen gas bubbles that form during the electrochemical reaction create a porous $\mathrm{Ni} / \mathrm{NiO}$ template after annealing. This porous mold can then be used to cast large scale PDMS to obtain PDMS micro-pillars with hierarchical shapes (Fig. 27(c)) [292]. In another approach, mushroom shaped elastomeric pillars were fabricated using a 2-photon lithography technique with UV-curable negative photoresist. [200]. Laser micromachining was used to fabricate molds to create wall-shaped poly-vinyl siloxane (PVS) adhesive structures [149]. An ultraprecision machining lathe equipped with a diamond tool was used to machine wedge-shaped structures on an aluminum mold that was used to fabricate wedge shaped PDMS adhesive structures shown in Fig. 27(a) [254].

Tip shape can be effectively manipulated using a variety of techniques. Mushroom shaped tips for pillars are commonly reported for gecko-inspired adhesives. The mushroom shape (Fig. 26(d)) can be obtained by developing an undercut during the fabrication of master mold negative. Undercut can be realized by using lift-of-resist (LOR) along with photoresist followed by selective etch of LOR [286] or by using a glass substrate with a double side exposure lithography $[128,268]$. The mushroom shaped pillars in PDMS were used to fabricate flexible polyurethane acrylate (PUA) negatives that can be used as patterned molds in a roll-to-roll process to yield flexible adhesive structures on UVcurable resin [285]. In another approach, uncured PDMS was applied selectively on the tips of the cured PDMS pillars and then cured while being pressed against a fixed substrate resulting in flattened tip ends (Fig. 26(c)) [201,270]. A stretching imprint process was reported to fabricate high aspect ratio PMMA pillars (Fig. 27(b)) using a micropatterned Teflon mold [144]. The tip shape can be controlled by changing the cooling speed during the stretching operation and the orientation of the pillars can be controlled by varying the lateral displacement of the mold during stretching [144].

Another material of interest for fabrication of dry adhesive structures is carbon nanotubes (CNTs) and Fig. 28 shows representative examples. Vertically aligned CNT arrays (VACNT) are being researched because of their smaller feature sizes and

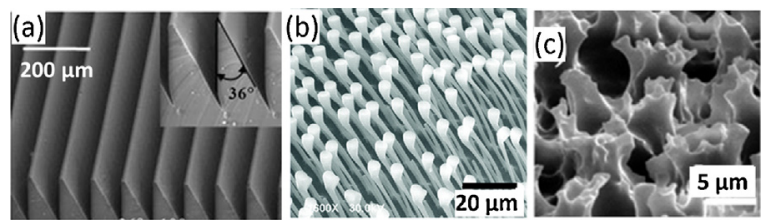

Fig. 27. Dry adhesive structures fabricated using (a) PDMS wedges cast from aluminum mold machined using a ultraprecision diamond tool [254] (reproduced with permission from John Wiley And Sons); (b) high aspect ratio PMMA pillars fabricated using a stretching imprinting process-capped pillars are obtained by fast cooling [144] (reproduced with permission of The Royal Society of Chemistry); (c) side view of hierarchical PDMS microarrays cast from a porous $\mathrm{Ni} / \mathrm{NiO}$ mold obtained using electrodeposition of nickel [292] (reproduced with permission of The Royal Society of Chemistry).

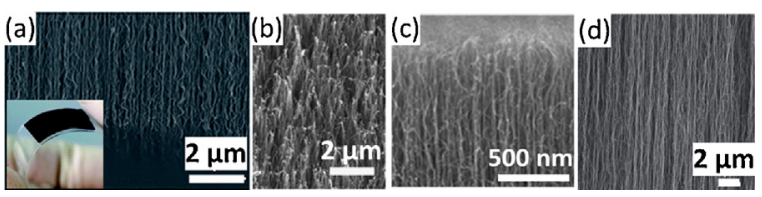

Fig. 28. CNT based dry adhesives; (a) VACNT array on flexible PET substrate fabricated by thermal CVD [178] (reproduced with permission of The Royal Society of Chemistry); (b) Vertically aligned double-walled CNT strands fabricated by low pressure CVD and bundled top nodes by plasma treatment [283] (reproduced under the Creative Commons Attribution 4.0 Generic license); (c) VACNT array synthesized by cold-wall CVD process [54] (reprinted with permission. Copyright 2015 American Chemical Society); (d) SEM image of spinnable VACNT arrays [62] (reproduced with permission of The Royal Society of Chemistry).

high aspect ratios that can lead hierarchical architectures and better conformal contact and higher adhesive strength than polymeric materials. CNT structures are also promising because of their superior controllable thermal, mechanical and electrical properties and their applicability in switchable adhesives as well as for fabricating flexible adhesive patches [62,129]. Typically, CNT based fibrillar structures are produced using a bottom-up manufacturing approach. Variations of chemical vapor deposition (CVD) including thermal CVD [178], low pressure CVD [283] and cold-wall CVD [54] have been utilized to synthesize VACNT arrays. Additionally, VACNT arrays with better alignment, narrower diameter distribution and higher density using CVD are also reported [62,63]. Apart from CNT-based fibrous structures, dry adhesive structures made up of polymethyl methacrylate (PMMA) and polyvinylidene fluoride (PVDF) fibers fabricated using electrospinning have also been investigated [226,227].

\subsubsection{Adhesive functional surfaces and related product applications}

Gecko-inspired adhesive structures are interesting for a wide variety of commercial applications such as robotics (climbing pads), space grippers and even for medical adhesive pads. From a practical application standpoint, easy detachment is as important as effective attachment. Thus, research effort is focused on creating 'switchable' dry adhesive structures where the adhesion can be controlled via external stimulus. Since the adhesive force is directly proportional to the total contact area between the dry adhesive structures and the counter surface, switching between the 'on' and 'off' stage can be achieved by changing the contact area.

Si nanowire arrays were fabricated by chemical etching and were modified using polymer brushes. Composite arrays based on poly 3sulfopropyl methacrylate potassium salt (PSPMA) can be controlled by changing the humidity while nanowire arrays with poly methacrylic acid sodium (PMAA) brushes show $\mathrm{pH}$ sensitive response [194]. A bio-inspired photocontrollable microstructured transport device (BIPMTD) was developed using an azobenzene containing a cross-linked liquid crystalline elastomer. A photoresponsive film is sand wiched between mushroom-shaped adhesive micropillars and PDMS backing layer [158]. When illuminated with UV light, the photoresponsive units change their shape and cause bending which leads to the detachment of the adhesive pillars as illustrated in Fig. 29(a). Contact area and thus adhesive force can also be manipulated by using anisotropic and directional attachment structures. PDMS pillars with large overhanging caps and steps were fabricated leading to anisotropic behavior. When the tips are dragged to the right, the contact area is increased, leading to strong adhesion [269]. The off state is achieved by dragging the pillars to the left where only part of tip makes contact (see Fig. 29(b) for illustration) [269]. In a similar approach, PDMS wedges switch from on- to offstate by application of shear force as shown in Fig. 29(c) [218]. In another approach, a soft gripper for pick and drop applications was developed containing an inflatable soft membrane covered with adhesive micro-pillars [245]. The inflatable membrane can be controlled via changing air pressure through a central tubing to control the adhesion. When the membrane is in a flat state created by low pressure, the adhesion force is stronger as high number of micropillars is in conformal contact with the object. When the membrane 


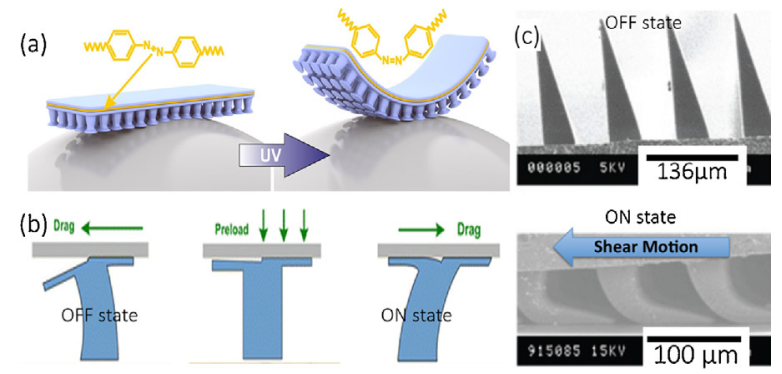

Fig. 29. Illustration of switchable gecko-inspired adhesive structures (a) the contact of the mushroom shape tips can be controlled by exposure to UV light [158] (reprinted with permission from AAAS); (b) pillar tips with anisotropic overhangs that change the contact area depending on the dragging direction from high adhesion 'off' state on left to low adhesion 'on' state on right [269] (reprinted with permission. Copyright 2016 American Chemical Society); and (c) wedge shaped adhesive structures that can be activated by application of shear force; in off state only the tips of triangular wedge makes contact thus creating low adhesion while the pillars bend to increase the contact area and adhesion when shear force is applied [218] ( 2013 IEEE. Reprinted with permission).

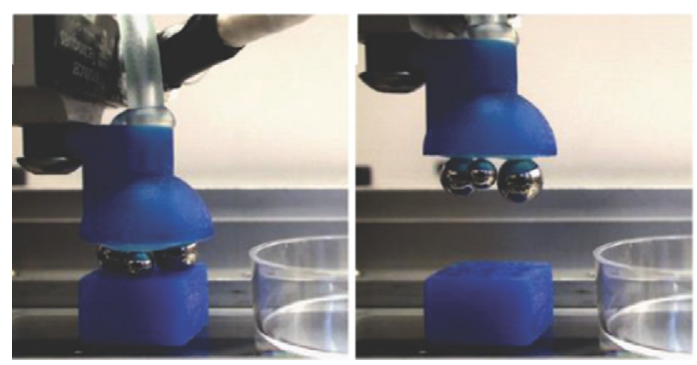

Fig. 30. Soft gripper containing a central tubing in 'pick and drop' experiment with spherical steel balls [245]. (reproduced with permission from John Wiley And Sons).

is inflated the number of pillars in contact reduces due to bending of the membrane leading to a low- adhesion state. The 'pick and drop' operation of soft gripper is depicted in Fig. 30 using steel balls as an example object.

Dry adhesives can also be potentially used for medical and surgical applications. A biodegradable and biocompatible tissue adhesive made of poly glycerol-co-sebacate acrylate was reported previously [196]. Similarly, a bio-compatible adhesive for tissue repair was developed from chitosan and showed excellent bonding capability [83]. A conductive patch was fabricated with a composite structure of gecko-inspired dry adhesive along with co-doped CNT and graphene. The conductive pad showed comparable adhesive forces to commercial wet adhesives. Furthermore, these pads have excellent stretching and bending properties while preserving their adhesive and electrical properties, making them suitable for application to human skin as an interface while serving as an ECG monitor (Fig. 31) [155]. Adhesive pads made from wedge-shaped PDMS adhesives discussed above have been used as shear adhesive grippers for space and other applications $[102,218]$. Shear adhesive grippers under zero gravity were successfully tested to grasp and release both flat and curved objects [142].

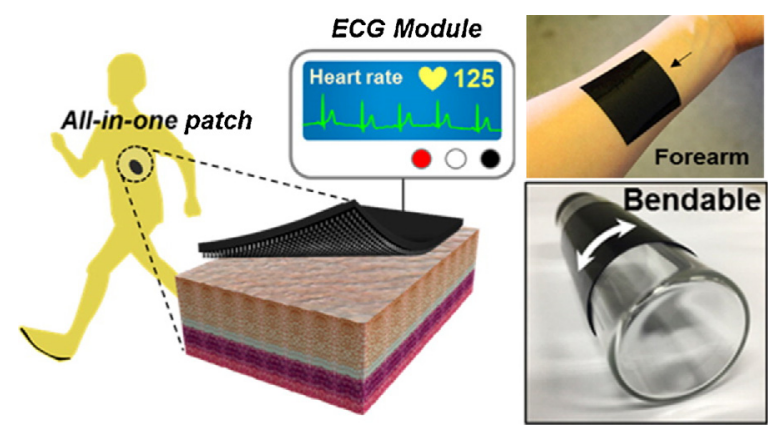

Fig. 31. Proposed use of flexible and bendable conductive dry adhesive patch conformally attached to human body [155] (reprinted with permission. Copyright 2016 American Chemical Society).
Gecko-inspired adhesive technologies have also been commercialized. Nanogriptech [111] have developed pressure-sensitive dry adhesive tape Setex ${ }^{\mathrm{TM}}$ along with custom gripping and fastening solutions. Another product Geckskin ${ }^{\mathrm{TM}}$ [119] combines the geckoinspired elastomeric adhesives with stiff fabrics to yield synthetic dry adhesive that can be repeatedly applied onto a variety of surfaces without leaving any residue. In summary, for the texture design, materials and process selection typical non-glue adhesive products demand scalable and low-cost processes for materials with flexibility, electro-mechanical multifunctionality and moldability with disposable property.

\subsection{Bio-inspired textures for controlled wettability}

Bio-inspired wettable surfaces (superhydrophobic, superhydrophilic, superoleophobic, superomniphobic and their combinations) have attracted significant attention due to their applicability in a variety of commercial applications such as self-cleaning coatings, water harvesting, antifouling, friction reduction, and others. As noted previously, physical and/or chemical textures are responsible for controlling the wettability of surfaces. This section is intended to discuss scalable and/or low cost manufacturing processes employed to replicate bio-inspired texture designs such as from lotus leaf and S. molesta.

The hierarchical arrangement of features along with their sizes plays a key role in fabricating lotus leaf inspired superhydrophobic surfaces along with hydrophobic surface chemistry as a material property of interest. Superhydrophobic structures have been fabricated on a broad range of materials and with a combination of manufacturing approaches as summarized in Fig. 32. A topdown manufacturing approach is typically used to process polymers, metals, alloys and semiconductor-based materials. These approaches include lithography/etching methods as well as laser surface texturing. Bottom-up manufacturing methods include sol-gel processing, colloidal assembly, CVD, and others addressing materials of interest such as metal oxide nanostructures, CNT, graphene, and more. A sequential manufacturing approach is typically employed to either fabricate a hierarchical texture (commonly achieved by bottom-up nanostructures grown on top-down manufactured micro-scale features) or for processing soft polymeric materials via molding/imprinting as well as for hydrophobic surface functionalization of textured surfaces. Table 1 illustrates some of the commonly reported processes, materials and texture designing methods. Due to space limitations, most commonly observed representative examples are chosen to highlight the use of different texturing methods to develop superhydrophobic surfaces with the desired micro structure size, distribution and hierarchy of features. More comprehensive examples for superhydrophobic structures can be found in following review articles and references therein $[6,27,288,94,143,153,167,193,244,272,284]$.

Apart from manipulating surface topography to achieve superhydrophobic effect, laser textured surfaces were also studied in vitro to selectively enhance cell adhesion for biomedical applications [66].

As discussed in the previous section, S. molesta shows superhydrophobic behavior with highly stable underwater air retention

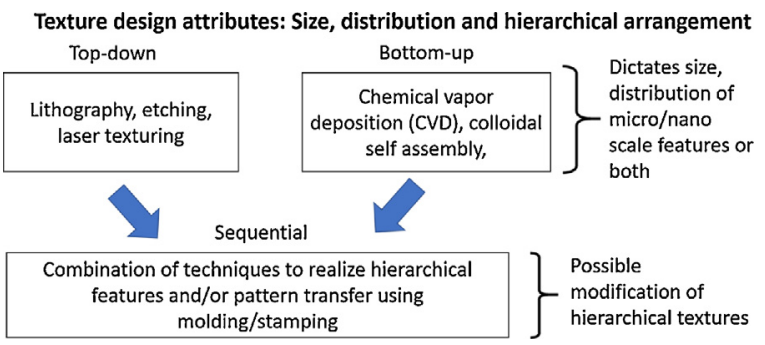

Fig. 32. Manufacturing approaches for fabricating bio-inspired textures with controlled wettability. 
Table 1

Manufacturing approaches for fabricating superhydrophobic textures.

\begin{tabular}{|c|c|c|}
\hline $\begin{array}{l}\text { Manufacturing method and } \\
\text { materials }\end{array}$ & Comments & Ref. \\
\hline $\begin{array}{l}\text { Top down: laser surface } \\
\text { texturing of copper surface }\end{array}$ & $\begin{array}{l}\text { Micron scale arrangement controlled } \\
\text { by the CAD model, submicron surface } \\
\text { morphology can be varied by } \\
\text { changing the scanning speeds }\end{array}$ & [187] \\
\hline $\begin{array}{l}\text { Top-down: wet etching of } \\
\text { copper substrate were } \\
\text { etched with } \mathrm{FeCl}_{3}+\mathrm{HCl} \\
\text { etchant with optional } \\
\text { oxidation using } \mathrm{NH}_{4} \mathrm{OH} \\
\text { solution }\end{array}$ & $\begin{array}{l}\text { Random micro-texture is obtained by } \\
\text { etching. The morphology and } \\
\text { distribution of features can be } \\
\text { controlled by varying etchant } \\
\text { concentration, etching time and } \\
\text { temperature and subsequent } \\
\text { oxidation }\end{array}$ & [82] \\
\hline $\begin{array}{l}\text { Bottom-up: spray coating and } \\
\text { curing expanded } \\
\text { polystyrene foam (EPF)/ } \\
\text { camphor soot composite } \\
\text { films on glass substrate }\end{array}$ & $\begin{array}{l}\text { Self-organized, random micro- } \\
\text { submicron sized structures are } \\
\text { obtained because of spray coating } \\
\text { followed by curing. The roughness } \\
\text { and morphology of features can be } \\
\text { controlled by changing the curing } \\
\text { temperature }\end{array}$ & [228] \\
\hline $\begin{array}{l}\text { Sequential: anodized } \\
\text { aluminum oxide mold was } \\
\text { used to imprint hierarchical } \\
\text { textures on polypropylene } \\
\text { films }\end{array}$ & $\begin{array}{l}\text { Anodization of micropatterned } \mathrm{Al}- \\
\text { coated mold. The submicron scale } \\
\text { feature size can be controlled by } \\
\text { changing anodization parameters } \\
\text { and/or subsequent } \mathrm{H}_{3} \mathrm{PO}_{4} \text { etching } \\
\text { parameters }\end{array}$ & [105] \\
\hline $\begin{array}{l}\text { Sequential: Ti6Al4V substrate } \\
\text { was sand-blasted followed } \\
\text { by hydrothermal growth of } \\
\mathrm{TiO}_{2} \text { nanowires }\end{array}$ & $\begin{array}{l}\text { Micro-scale features can be } \\
\text { controlled by size of abrasive media } \\
\text { used during sand-blasting. } \\
\text { Submicron features can be controlled } \\
\text { by hydrothermal growth temperature } \\
\text { and time }\end{array}$ & [239] \\
\hline
\end{tabular}
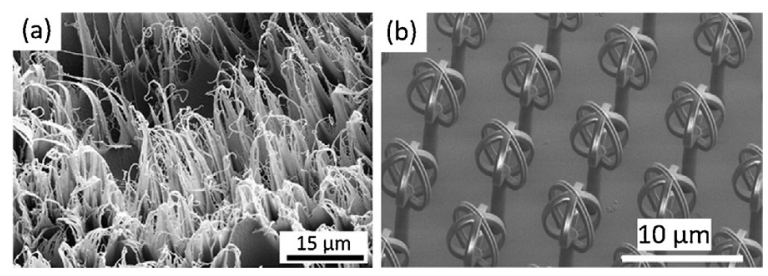

Fig. 33. (a) Polymer nanofur fabricated by hot pulling [150] (reprinted with permission. Copyright 2015 American Chemical Society); (b) egg-beater morphology fabricated by laser lithography and 2-photon polymerization [258] (reproduced with permission of ACS Publications).

and can be potentially useful for applications such as drag reduction. Air retaining superhydrophobic structures were realized using vertically aligned carbon nanotubes (VACNT) grown by CVD technique. The hydrophilic tips of CNT were due to presence of carbonaceous nanostructure [207] or because of $-\mathrm{OH}$ groups on regrown CNTs [11]. In another approach, polycarbonate nanofur were hot pulled from sandblasted steel plates (Fig. 33(a)) to fabricate air retaining hairy structures [150,225]. Egg beater shaped hairy hydrophobic structures were also reported (Fig. 33 (b)) by using hydrophilic photoresist patterns developed using direct 3-D laser lithography and two photon polymerization [258].

Alternative hydrophobic/hydrophilic domains inspired from the desert beetle are also being researched for fog harvesting applications [297]. A chemical modification was reported to a superhydrophobic state using heptadecafluorodecyl-trimethoxysilane (FAS) followed by patterned UV exposure to fabricate star-shaped superhydrophilic domains [12]. In similar approach, direct laser writing was employed to create patterned domains on $\mathrm{TiO}_{2}$ surface for water collection [266]. Using another method, steel needles were punctured through hydrophobic polymer-silica nanocomposite to create nucleation sites for droplet formation and growth [203].

\subsubsection{Controlled wettable functional surfaces and related product applications}

Bio-inspired superhydrophobic/hydrophilic surfaces have inspired a variety of product applications because of their wide applicability. Relevant product applications involving drag/friction reduction, antifouling, and their combination with AR coatings are discussed in the following sections.

Bio-inspired superhydrophobic surfaces have inspired many technologies with applications in self-cleaning paints (Lotusan) [248], textiles [7] and biomedical field [240]. The silica-based water and oil repellent coating GreenShield ${ }^{\mathrm{TM}}$ was developed by BigSky Technologies [120]. Superhydrophobic metallic structures fabricated by laser surface texturing are being investigated under the TresClean project [126]. Similar hydrophobic textured coatings have also been developed by ARLD [109] and Lotusleafcoatings [110]. Another company NBD Nano has developed anti-icing, superhydrophobic glass coatings as well as beetle-inspired fogharvesting coatings [112]. In summary, texture design, materials and process selection for typical products with superhydrophobic, superhydrophilic, superoleophobic, superomniphobic and their combination properties demand region selectivity, massive scalablility and ultra low-cost processes for materials with flexibility as well as stiffness, chemical affinity but corrosion resistance and moldability with disposable property.

\subsection{Bio-inspired textures for hydrodynamic and boundary lubrication}

To increase the efficiency of machines as well as manufacturing processes, it is important to reduce energy losses. Frictional losses are one of the primary causes of concern in the majority of mechanical systems. It has been estimated that almost $30 \%$ of fuel is utilized in overcoming frictional losses in vehicles such as passenger cars, buses and trucks [107,108]. Thus, developing effective surface designs and dry as well as wet lubrication strategies are critical in combating these challenges. Fig. 34 depicts three distinct stages of lubrication and the corresponding friction as a function of the lubricant parameters and operating conditions. On the right extreme, in a hydrodynamic regime, the friction coefficient starts to rise because of the hydrodynamic drag of the lubricant even though the two surfaces are not in direct contact. The friction goes up in a boundary lubrication regime (left extreme) because the two surfaces are in direct contact and the applied load is dissipated through the asperity contact.

As described in Section 2, nature has developed multifunctional surfaces for friction management making use of specific textures for species' habitat environments. Examples span from marine animals such as sharks where hydrodynamic friction drag is manipulated via riblet structures -to- biological lubricant fluids such as those observed in the toes of tree frogs and lizards -to- the energy management under boundary conditions observed in the limbless locomotion of snakes [185]. This section is focused on the design and manufacturing processes for bio-inspired surfaces and strategies to reduce frictional losses and is divided into two parts (1) textures for hydrodynamic drag reduction and (2) textures for boundary and dry lubrication.

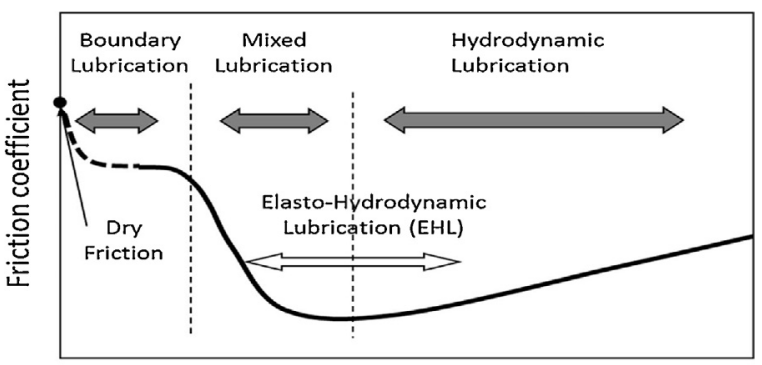

Lubrication parameter, $\mathrm{\eta V} / \mathrm{P}$

Fig. 34. Stribeck curve illustrating the relationship between friction coefficient, lubricant properties and operating conditions [164] (reproduced under the Creative Commons Attribution 3.0 Generic license). 


\subsubsection{Surfaces for hydrodynamic drag reduction}

4.3.1.1. Manufacturing processes and approach. Shark skin is an excellent example of a multifunctional surface as it can be used as a model for developing drag reduction schemes and low friction (tribological applications) surfaces along with antifouling surfaces as discussed in the next section. Hence, some of the manufacturing processes discussed in this section will also overlap with manufacturing approaches discussed in Section 4.4.

The pattern on skin of fast swimming sharks can be simplified into a 2-D riblet type structures and can be produced using variety of top-down manufacturing processes as the features' sizes are typically of the order of micron scale. The size and distribution of the features can be effectively controlled during the material removal processes either by controlling the movement of the tool during machining or by defining the pattern using lithographic techniques. A roller embossing process was utilized to fabricate shark-skin inspired micro-riblet pattern on soft polymeric materials such as polyvinyl chloride (PVC) and polyethylene terephthalate (PET) [95]. A simplified micro-riblet pattern was first created on $\mathrm{Ni}$ roller mold using a Ultra-Violet Lithographie Galvanoformung Abformung (UV-LIGA) process and then applied on polymer films (Fig. 35(a)). In another attempt, a hierarchical texture was fabricated on $\mathrm{Ni}$, containing nanostructures on microriblets using laser interference lithography, nanoimprinting and pulse-reverse-current electrochemical deposition [156]. Similarly, use of a wire EDM process was reported to fabricate a simplified micro-riblet pattern [214]. Micro-riblet structures fabricated on compressor blades using grinding techniques were also investigated and optimized to improve geometrical accuracy of microfeatures [67,68]. In another approach, bio-inspired shark skin containing rigid denticles on flexible membrane substrate was realized using 3-D printing. A micro-CT scanner was used to scan the skin of a Mako shark and a representative skin denticle was picked and used to create a 3-D model. The denticle model was then duplicated and arranged to form a denticle array to create a final 3-D object, which was printed using a 3-D printer with multiple nozzles to print both rigid and flexible regions as shown in Fig. 35(b) [273]. Apart from shark skin inspired geometries, the $S$. molesta inspired texture described in previous section is also being investigated for development of drag reducing structures due to effective pinning of water-air interface $[15,131]$, which results in reduction of skin friction.

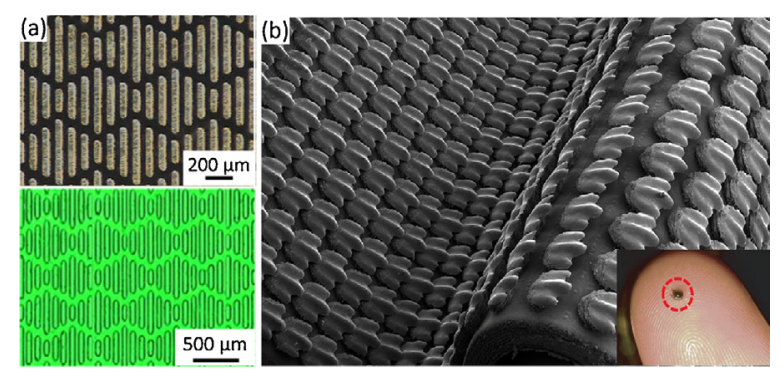

Fig. 35. (a) (top) Nickel mold with micro-riblet pattern fabricated by UV-LIGA process and (bottom) image of PET film and with shark-skin-inspired micro-riblets [95] (reproduced by permission of the Institution of Engineering \& Technology); (b) 3-D printed rigid denticles on flexible substrate membrane and single synthetic denticle of $1.5 \mathrm{~mm}$ length on a human finger (inset) [273] (reproduced with permission from Journal of Experimental Biology).

4.3.1.2. Drag reducing functional surfaces and product applications: The micro-riblet patterns discussed above were tested for their drag reduction properties under various conditions. The metallic hierarchical textures fabricated using laser interference lithography were tested in a turbulent flow regime and showed a maximum drag reduction of $36 \%$ compared to an untextured surface [156]. Similarly, the 3-D printed riblets have demonstrated about an $8.7 \%$ drag reduction in low speed conditions [273]. Up to
$10 \%$ reduction in wall friction was reported for riblet structures fabricated using grinding process on compressor blades [67]. Similar riblet structures also contributed to faster swimming speeds and a reduction of swimming energy under kinematic tests [273]. Shark skin fabricated by a bio-replication method (discussed in Section 4.4) also showed an improvement in drag reduction properties [190,192]. Apart from shark skin inspired textures, $S$. molesta inspired nanofur also resulted in improved drag reduction properties [150].

Drag reduction coatings can be utilized to reduce fuel consumption in marine and air transport vehicles. A research project FAMOS conducted by Lufthansa airways is experimenting to develop sharkskin inspired coating on aircraft. The durability and efficiency of the coatings has been successfully demonstrated during testing and the coating is expected to be launched on commercial aircraft in the near future [124]. Sharkskin has also inspired swimsuits that enable faster speeds due to drag reduction and have been introduced into the market by several corporations, for example Speedo corporation under the brand name FASTSKIN [118].

\subsubsection{Surfaces for boundary lubrication related friction manipulation}

Surface texture has also been widely studied for developing effective boundary lubrication strategies [75]. It is widely recognized that texture aids lubrication via the following effects: (1) decreasing the actual contact area; (2) serving as a reservoir for supplying lubricant as well as for capturing wear debris; (3) aiding the formation of lubricant film and aid the fluid flow through the channels [37]. This section is focused specifically on bio-inspired surfaces developed for tribological applications. It is also important to note that the tribological processes and phenomena are highly stochastic and thus the optimized design of surface texture for improved tribological response is also very specific to the nature of application, mating materials, lubricant properties and intended application.

4.3.2.1. Manufacturing processes and approach. The size of surface features and distribution of features along with their directionality are key design components for developing bio-inspired surfaces for friction and wear management as previously discussed. These texture attributes together with material properties such as elasticity and hardness govern the tribological response. Top-down manufacturing processes such as lithography and etching as well as laser surface texturing (LST) are commonly used to fabricate bioinspired textured surfaces as the size and distribution of micron scale features can be easily replicated with precision. Polymeric samples can be prepared by using molding technique from micropatterned molds that are typically created using lithography and etching methods. Similar bio-inspired texture patterns fabricated using micro-milling setup on hardened tool steel were also reported and tested under dry and lubricated conditions [255].

A snake-skin inspired texture as shown in Fig. 36(a) was fabricated on 100Cr6 bearing steel pins by using a Q-switched ytterbium fiber laser [93]. Directional features were created in epoxy resin via a two-step molding process using negative polyvinylsiloxane (PVS) molds from biological samples $[18,92] . \mathrm{TiO}_{2}$ masks were utilized to fabricate textured Ti6Al4V
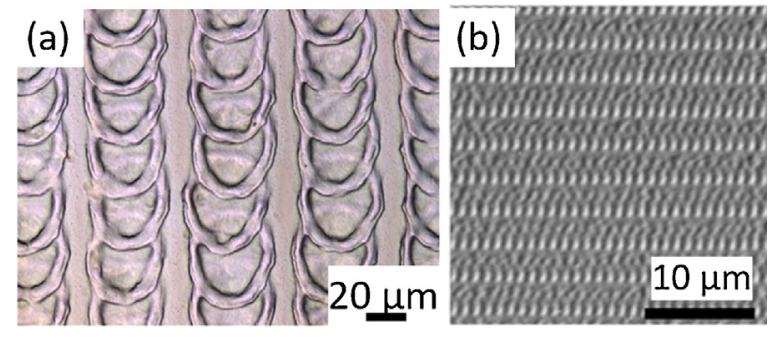

Fig. 36. A snake-skin inspired surface textures fabricated by (a) laser surface texturing of 100Cr6 steel [93] (@ IOP Publishing. Reproduced with permission. All rights reserved); (b) 2-step molding with PVS negative molds [18] (reproduced under the Creative Commons Attribution 2.0 Generic license). 


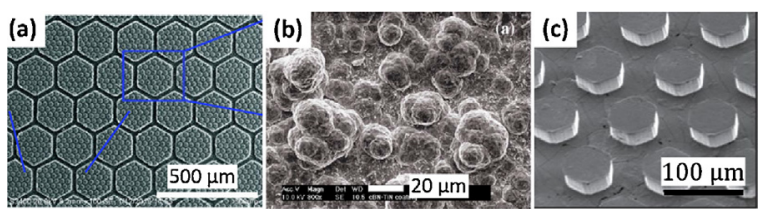

Fig. 37. (a) Hexagonal textures in PDMS fabricated from lithographically etched Simasters [176] (reprinted with permission. Copyright 2015 American Vacuum Society); (b) lotus-leaf like cBN-TiN coating fabricated by ESC-CVI [278] (reprinted with permission from Elsevier); (c) hexagonal textures in PVS from a Si-master [259] (reproduced under the Creative Commons Attribution 4.0 Generic license).

alloy pins employed as a part of the bio-compatible Ti6Al4VUHMWPE pair used as artificial hip joints [61].

Bio-inspired textures for friction management in engineered systems have also been inspired by the toe pads of tree frogs, crickets and newts as well as shark skin and lotus leaves as depicted in Fig. 37. Lithographically patterned masters were used to fabricate hexagonal pattern on curable polymers such as PDMS $[130,176]$ and PVS [259,263]. A lotus leaf like texture was created on cBN-TiN composite coating by using an electrostatic spray coating (ESC) followed by chemical vapor infiltration (CVI) [278] and overcoating of molybdenum di-sulphide $\left(\mathrm{MoS}_{2}\right)$.

4.3.2.2. Lubrication management control functional surfaces and product applications. Textures for friction management can be used for both reducing friction as well as increasing friction depending on the intended use and application environment. Lotus leaf inspired cBN-TiN coatings have shown major friction reduction under dry conditions when tested with a $\mathrm{MoS}_{2}$ solid lubricant embedded in textured micro-reservoirs [278]. Similarly, the snakeskin inspired surface textures described above showed a reduction in friction as compared to untextured control samples under dry conditions [17,93]. However, under wet lubricated conditions with additive free mineral oil, friction was higher than with smooth surface [93]. Similar observations were made for hexagonally patterned substrates inspired from tree frogs where hexagonal textured PVS pads were applied on razor blades and their performance was tested under shaving foam [259]. The hexagonal patterned structure allows higher contact area between the razor and stretchable skin. Also, at the same time, the micro-channels among the hexagonal pads allow the effective drainage of the fluid avoiding any hydrodynamic gliding. As a net result, researchers have enhanced the efficiency of the razors through this bioinspired engineering [259]. LST is being widely investigated as a texturing strategy to reduce friction in many industrial and biomedical engineering products such as piston rings, engine liners, hip joints, and others. However, the majority of the research is based on curiosity driven approaches involving specific material (s) and lubricant(s), a process unlike the one that natural organisms have used to develop superior surface structures via evolution that are environment specific. Scale like features found on snake skin and shark skin are directional and dynamic as the orientation of the scales can be manipulated by the active motion of the snakes and sharks. This result can be utilized to develop adaptive locomotion strategies, a future direction for artificial engineered systems. In summary, the texture design, materials and process selection for typical products with adaptive friction and wear properties demand scalablility and occasionally ultra lowcost processes for materials typically with abrasion resistance from operating environment and corrosion resistance.

\subsection{Bio-inspired textures for antifouling $(A F)$}

Biofouling can be defined as the undesirable accumulation of biological micro- and macro-scale organisms $[32,198]$ and it poses a severe problem to water submerged vehicles and equipment such as ships and submarines as well as for medical and surgical devices [195]. The use of chemical agents and paints to reduce biofouling is discouraged because of their potential harm to the environment. Hence, it is necessary to develop environmentally friendly alter- natives to avoid biofouling [208]. Surface topographies inspired from underwater species such as sharks and seashells [195] provide a promising strategy to develop environmentally friendly antifouling solutions and will be discussed in this section.

\subsubsection{Manufacturing processes and approach:}

Key design considerations for fabrication of antifouling (AF) structures have been discussed in previous sections. Especially, as per Section 4.3.1.1, shark skin inspired riblet textures for antifouling applications have been fabricated via several top- down machining techniques such as the lithographic techniques used for fabricating the Sharklet $\mathrm{AF}^{\mathrm{TM}}$ micropattern [236,235]. However, these approaches are mainly suitable for creating simplified 2-D shark skin like features and fail to capture the intricate 3-D detail of shark skin topography [191]. Hence, direct bio-replication methods are being investigated to fabricate synthetic shark-skin topographies. A typical fabrication approach is summarized in Fig. 38.

Bio-replication methods to fabricate AF structures use curable polymers such as PDMS [55,220] or silicone rubber [190] to replicate shark skin pattern and form a negative mold. It is important to clean and pre-treat the shark skin before forming a mold in order to remove mucus, moisture and any contaminants on the skin surface, which could lead to defects in the mold [55,190]. A positive replica of the shark skin can then be obtained from the negative mold using micro-imprinting methods. Curable soft polymers and resins are typically used to fabricate positive replicas from the PDMS molds either by imprinting or molding techniques. A similar strategy has also been adapted to fabricate AF structures inspired from rice leaves, white crab and Mollusk shells [13,36,56]. Fig. 39 shows AF replicas prepared by bio-replication methods using direct bio- templating techniques.

It is also important to manufacture bio-replicated and bioinspired riblet AF patterns on a large scale for their use in target industrial applications. Thus, different manufacturing strategies are being explored and promising results have been reported as discussed below. A roller-based continuous fabrication approach has been adopted using a bio-replicated using AW-01 epoxy resin and silicone rubber [290]. The biological shark skin is first modified using sputtering and a lithography technique to make it suitable for pasting on a roll. The modified shark skin roller can then be used to create a continuous negative template on an AW-01 resin, which was used to cast silicone rubber into positive shark skin replica [290].

Texture design attributes: Size, distribution, shape and orientation

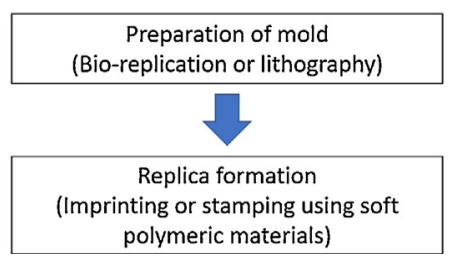

Fig. 38. Processing flow chart for manufacturing AF surface structures or coatings.
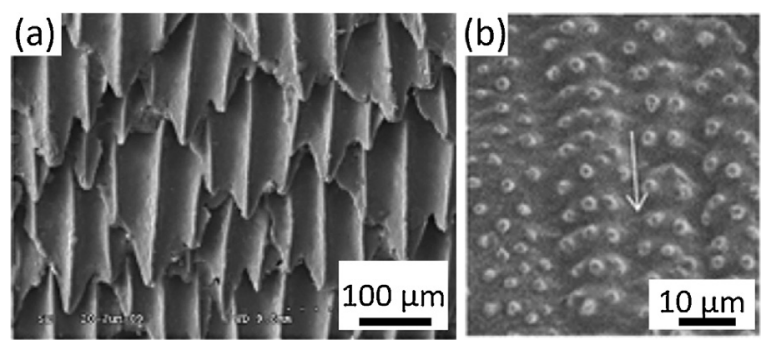

Fig. 39. (a) Replicated shark-skin coating formed on UV-curable resin polyurethane diacrylate (PUC) using PDMS negative mold using a UV transparent 3-wheel embossing belt setup [55] (reprinted with permission from Elsevier); (b) liquid urethane polymer replica of rice leaf texture formed using a two-step soft lithography molding using a silicone rubber mold [35] (reproduced with permission of The Royal Society of Chemistry). 


\subsubsection{Anti-fouling functional surfaces and related applications}

This section is focused on discussion of antifouling strategies inspired from sharkskin and other examples, unlike the previous section which highlighted the drag reduction and tribological functionality for engineered applications.

The bio-replicated surface textures described above were tested for their antifouling response and showed enhanced performance [220]. Fig. 40 shows a comparison of smooth PDMS and microtextured sharkskin replica obtained by soaking samples for 70 days in the Pearl river, China and subsequent removal and washing. The sharkskin replica surface shows a superior antifouling response where the fouling algae species are loosely attached and get washed away easily as seen in image [220]. Similarly, another group tested the effect of surface morphology on the fouling response of algae using a UV-curable resin PUC [55]. It was noted that sharkskin surface morphology shows remarkable anti-fouling properties as compared to smooth and micro-grooved texture formed on the same material. White crab replica texture also showed effective anti-fouling response as compared to smooth samples when tested using settlement assays of Closterium and Navicula algae [56]. Anti-fouling response of shell replica molds was studied using different resin materials and it was found that E44 resin better replicates the shell surface topography and better resists the attachment of fouling organisms [13]. Thus, material properties, physical and chemical, in terms of replicating the pattern on a biological surface ultimately dictates the desired response.

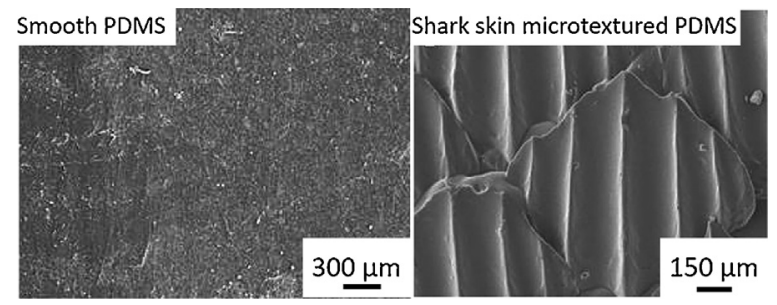

Fig. 40. Comparison of SEM images of fouling tests performed on smooth PDMS and shark skin microtextured PDMS with algal cells by soaking in a river after removal and washing [220] (reproduced under the Creative Commons Attribution 4.0 Generic license).

Apart from shark skin replicas, shark skin inspired micro topographies have been widely studied for their antifouling properties. Most notably, coatings developed by Sharklet technologies have been used for a variety of submarine and medical applications [125]. The patterned coating was applied on a variety of medical instruments such as stethoscope covers (Stethoskin ${ }^{\mathrm{TM}}$ ) and showed resistance to bacterial growth [113]. Another company, Micanti [115] has developed textured coatings (Thorn- ${ }^{\mathbb{R}}$ ) made from nylon fibers that can be applied on ships to enhance antifouling as well as fuel efficiency [41]. Anti-fouling is relatively understudied for engineering applications and has significant realworld opportunities. Also, regenerative textures are essential for this application as textures are likely to dull due to wear from operating environment. In summary, the texture design, materials and process selection for typical products with tailored AF properties demand massive scalablility and ultra low-cost processes for materials typically with abrasion resistance from operating environment and corrosion resistance.

\subsection{Bio-inspired textures for structural colors (photonics)}

Many natural systems such as butterfly wing and peacock feather show vivid and beautiful structural colors. The observed colors are due to the selective absorption of certain wavelength(s) as incident light interacts with a physically textured (structured) material with a varying refractive index, a key material property for design consideration. Since the color originates from only the physical effects and not from the pigments, structural colors have several key consumer and strategic applications such as displays, security coding, clothing, cosmetics and more.

\subsubsection{Manufacturing processes and approach:}

Structural colors can be obtained by creating photonic structures with an alternating refractive index in a 1-D or 2-D or 3-D network. As evident from Fig. 20, the complexity of photonic structures increases from 1-D periodic structures -to- 3-D periodic structures. Accordingly, the manufacturing approaches also vary depending on the complexity of the structure and end applications. This section summarizes manufacturing approaches and some of the commonly reported fabrication techniques with suitable examples of 1-D, 2-D and 3-D periodic structures. 1-D and 2-D structures can typically be realized using top-down manufacturing processes whereas bottom-up and sequential manufacturing approaches are mainly used for fabricating desired 2-D and 3-D photonic structures. Patterning (that controls size and periodicity of features) can either be performed using lithographic tools or by the thermodynamic self-assembly of colloidal particles. Like antireflective structures (Section 4.6), the size of features should be of the order of the wavelength of incident light. Hence lithography/etching along with self-assembled colloidal particles are employed as the size and periodicity of features can be effectively controlled, as required. These approaches are summarized in Fig. 41.

Morpho butterfly inspired lamellar structures are fabricated using lithography. A research effort reported alternate layers of PMMA/lift of resist (LOR) to fabricate lamellar structures with branches [296,295]. A multilayer assembly was patterned using ebeam exposure. An alternating development/dissolution process led to the formation of branch-like structures by creating undercuts in the LOR layer. The length of the undercut can be controlled by varying the etching time as shown in Fig. 42(c). In another approach, the branch like structure was obtained by using laser interference lithography with dual beam exposure with the help of a reflective layer deposited under the photoresist layer [242]. 1-D grating structure was fabricated in amorphous Si with a nanoimprint lithography process by using PMMA resist and a $\mathrm{SiO}_{2}$ mold [172]. By changing the widths of the grating structures, assorted colors were realized as seen in Fig. 42(a) [172]. In another approach, 1-D gratings were fabricated on Cu substrates via laser ablation technique. A picosecond laser with a $1064 \mathrm{~nm}$ wavelength [188] as well as a femtosecond Ti:Sapphire laser with a $800 \mathrm{~nm}$

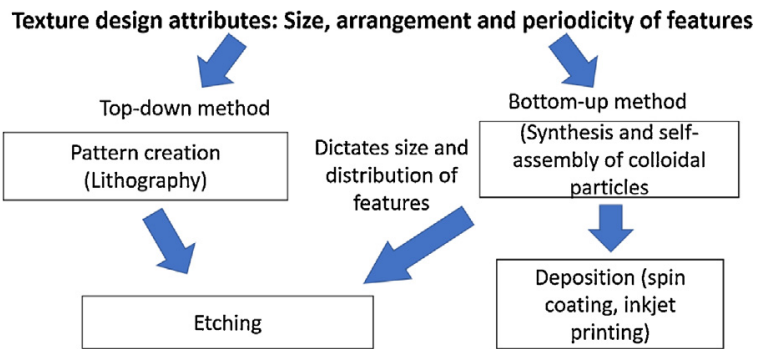

Fig. 41. Processing flow chart for manufacturing bio-inspired structural colors.
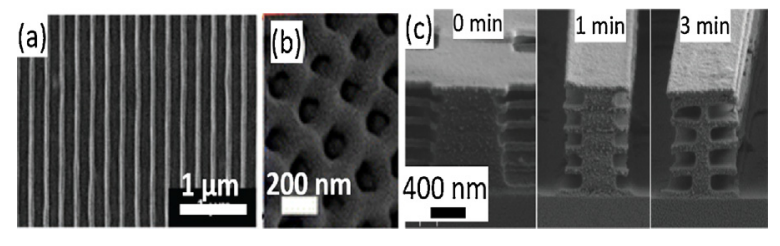

Fig. 42. Structural color fabrication by top down approaches: 1D patterned amorphous silicon (a-Si) gratings of $50 \mathrm{~nm}$ width and $220 \mathrm{~nm}$ period for color filter application [172] (reproduced with permission from John Wiley And Sons); (b) $\mathrm{TiO}_{2}$ nano-bowl arrays prepared by anodization [260] (reprinted with permission. Copyright 2016 American Chemical Society); (c) Butterfly type architectures formed using PMMA/LOR layers, undercut lengths in LOR layer as function of etching time [296] (reproduced with permission of The Royal Society of Chemistry). 


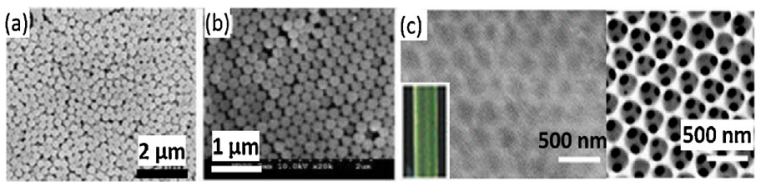

Fig. 43. Structural color fabrication by bottom up approaches: (a) SEM image of structural colored films formed by inkjet printing CdS spheres [279] (reprinted with permission. Copyright 2017 American Chemical Society); (b) SEM image of colloidal crystal films formed by gravity sedimentation and self-assembly of silica nanoparticles [87] (reprinted with permission from Elsevier); (c) GelMA hydrogels prepared from inverse opal scaffold (right) and resultant structural colored hydrogels (inset) [84] (reprinted with permission from PNAS).

wavelength [183] were used to fabricate periodic surface structures. 2-D periodic structures have also been fabricated using top-down manufacturing processes. A silver-silica-silver (MIM) meta-surface containing a triangular 2-D lattice of periodic holes on top of a silver layer was fabricated using a one-step focused ion beam milling [58]. Peacock feather like photonic structures were prepared by using anodization and etching with $\mathrm{TiO}_{2}$ [260]. The anodization potential controls the size of nano-bowl/pore (Fig. 42 (b)) and ultimately controls the resultant color.

Colloidal assembly of nanoparticles offers an attractive alternate strategy to fabricate photonic structures (Fig. 43), especially for making 3-D photonic structures. In addition to providing flexibility in the manufacturing of a variety of structures, such solution-based processing also allows for photonic structure coatings for potential textile applications and inkjet printing. The arrangement of particles and thus the resultant color can be controlled by controlling process variables such as particle size, concentration and curing time. Structural colors were realized in colloidal crystal (CC) thin films by controlling the size of silica nanoparticles (SiNP) during synthesis [87]. The films were formed by a gravity sedimented self-assembly of nanoparticles and drying from a colloidal suspension (Fig. 43(b)). Inkjet printing using CdS nanosphere ink was used to print structural colored films on paper (Fig. 43(a)). CdS nanosphere size was controlled by changing the concentration of the precursor during synthesis [279]. Use of sacrificial CC template of SiNP is discussed to realize structurally colored hydrogels [84]. A pregel solution of methacrylated gelatin (GelMA) was infiltrated into the nanopores of a dried self-assembled SiNP network and then polymerized. The SiNPs were then etched using HF acid solution resulting in inverse opal structured hydrogels (Fig. 43(c)) [84]. A combinatorial approach involving quasi-ordered $\mathrm{ZnO}$ nanostructures was demonstrated. [154]. The resultant color was controlled by adjusting the growth times for $\mathrm{ZnO}$ on a seed layer. The seed layer can also be masked and patterned for selective growth and etching to create the ability to control the domains of desired colors [154].

\subsubsection{Structural colored functional surfaces and product applications}

Bio-inspired structural colors have major applications including filters, displays, cosmetics and security. They can also be used for aesthetic purposes such as painting or for low-cost approaches for replicating images. Films and coatings can also be applied for creating colored fabrics for dye-free textiles. This subsection gives an overview and outlook of structural color product applications.

1-D gratings (Fig. 42(a)) and 2-D meta-surfaces can be used as reflective [172,241] or subtractive [213] color filters. The color can be tuned by adjusting the size and period of the surface features. 2-D color filters have also been developed using plasmonic metal-dielectric structures with silver [241,251] and aluminum [72] contacts [139].

Photonic crystal structures formed with colloidal crystals can be used to create pigment-free fabrics. Use of self-assembled SiNP (Fig. 43(b)) was demonstrated to successfully coat cotton and nylon fabrics with different colors [88,89]. In another approach, a mixture of colloidal poly-styrene-methyl methacrylate - acrylic acid (P(St-MMA-AA)) particles, carbon black and polyacrylate was demonstrated to develop a spray coating technique for coloring cotton fabrics. The coated fibers show a range of colors (Fig. 44(a)) depending on the size of microspheres and the color did not show

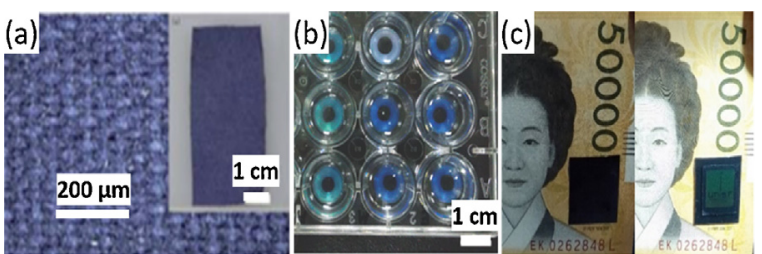

Fig. 44. Applications for structural colors: (a) colored fabrics obtained by spray coating P(St-MMA-AA) nanoparticles of sizes $217 \mathrm{~nm}$ [291] (reproduced under the Creative Commons Attribution 3.0 Generic license); (b) colored hydrogel contact lenses fabricated from inverse opal scaffolds created with $\mathrm{SiO}_{2}$ nanoparticles [281] (reproduced with permission from John Wiley And Sons); (c) anti-counterfeiting applications of monolayered silica films fabricated by inkjet printing showing covert-overt transformation of the printed image under bright illumination [206] (reproduced under the Creative Commons Attribution 4.0 Generic license).

any deterioration even after 10 washing cycles [291]. P(St-MMAAA) was also used to obtain dye-free flexible colloidal fibers using electrospinning [289].

Structural colors can also be used for anti-counterfeiting applications. Monodispersed SiNP [206] and CdS [279] particle (Fig. 43(a)) films were applied using inkjet printing those are light incident angle and illumination sensitive. These can be used as a tamper-proof seal as illustrated in Fig. 44(c). Nanotech security corp. [117] have developed KolourOptik ${ }^{\circledR}$ technology with e-beam lithography based molds that can be used in a roll-to-roll or direct stamping method for fabricating anti-counterfeit banknotes and tax stamps. Morphotonix have also developed injection molding based structural color templates that can be used to fabricate custom-made secure plastic seals [116]. Similarly, holographic structures manufactured by embossing techniques on polymeric and sheet metal substrates have also been investigated for anticounterfeiting applications [21,221].

In another application, structurally colored hydrogels with inverse opal morphology were used to create colored contact lenses as seen in Fig. 44(b) [281]. A team reported use of a 3-D printed dendritic block copolymer (BCP), where the phase separation of $\mathrm{BCP}$ at the nozzle of 3-D printer due to localized heating creates different surface morphology resulting in structural colors [39]. The color can be tuned by changing the molecular weight $\left(\mathrm{M}_{\mathrm{w}}\right)$ of BCP. Hollow tubes fabricated from such 3-D printed textured architectures can be used as light guides. Structural colors are also finding their way into the cosmetics consumer market as a potential solution to create pigment free makeup [114]. In realizing structural colors and AR coatings by design one must consider combination of optically sensitive and active or passive soft materials along with particles with or without texture patterns. Spatial precision and redundancy have been observed to be key for achieving application specific texture design(s). Manufacturing processes desired for most of these applications demand massive scalability along with environmental sustainability for disposal of applied materials.

\subsection{Bio-inspired textures for anti-reflection (AR)}

AR structures and coatings are important for a wide variety of photovoltaic and optoelectronic applications such as solar cells, lasers, flat panel and flexible displays and light emitting devices. Multilayer films of alternate high-low refractive index and subwavelength structures (SWS) are the two primarily used strategies to achieve AR structures. Bio-inspired, moth-eye mimicking SWS are promising when compared with the multi-layer stacks because of their broadband application window (multi-layer stacks are deigned to have AR property in a limited range of wavelengths) and superior wide-angle response $[43,47,179]$. These types of AR structures can either be created on the substrate or be applied in the form of a textured coating. Some of the common manufacturing processes and applications for bio-inspired AR textures are discussed below.

\subsubsection{Manufacturing processes and approach:}

The critical design aspects for effective moth-eye inspired structures, as discussed previously, are guided by size (sub- 
micron), shape and the periodic arrangement of nano-nipples. Both top-down and sequential manufacturing approaches can be utilized to fabricate SWS. Top-down manufacturing approaches consisting of patterning, lithography and etching are commonly utilized as they allow process flexibility in terms of materials and the process control necessary to fabricate precise feature sizes and achieve the desired periodicity. Additionally, this approach aligns well with a variety of materials and respective target applications such as solar cells and optoelectronics. Apart from achieving the required pattern via lithography, a variety of self-assembly techniques can also be utilized to create the periodic pattern that can be followed by selective material removal to achieve SWS. Another sequential manufacturing approach includes the use of top-down techniques to create SWS molds that can be used to process a variety of polymeric materials. Fig. 45 summarizes the above approaches and highlights the typical process methodology to fabricate AR structures and coatings.

Self-assembled nanoparticles have been used as etching masks to create SWS structures on a variety of substrate materials. Polystyrene (PS) nanoparticles are used to create pillars and cones or different heights on transparent substrates such as glass substrate [140] and quartz [141] as well as on $\mathrm{SiN}_{\mathrm{x}}$ film [287]. Si-masters were fabricated using a PS monolayer and Reactive Ion Etching (RIE) with a $\mathrm{SF}_{6}, \mathrm{C}_{4} \mathrm{H}_{8}$ and $\mathrm{O}_{2}$ gas mixture [104]. The height and shape of pillars can be controlled by controlling the size of the nanoparticles and etching time. Similarly, colloidal silica particle assemblies have also been utilized as etching masks to create SWS on Si $[90,104,249]$ and CdTe [52] substrates. Plasma etching using $\mathrm{CH}_{4} / \mathrm{H}_{2} / \mathrm{Ar}$ was used to obtain CdTe nanopillars shown in Fig. 46(b). After the colloidal selfassembly of particles in a hexagonal array, an additional etching step with $\mathrm{CF}_{4} / \mathrm{Ar}$ isotropic RIE can be employed to reduce the size of nanoparticles mask to manipulate the size and periodicity of etched features.

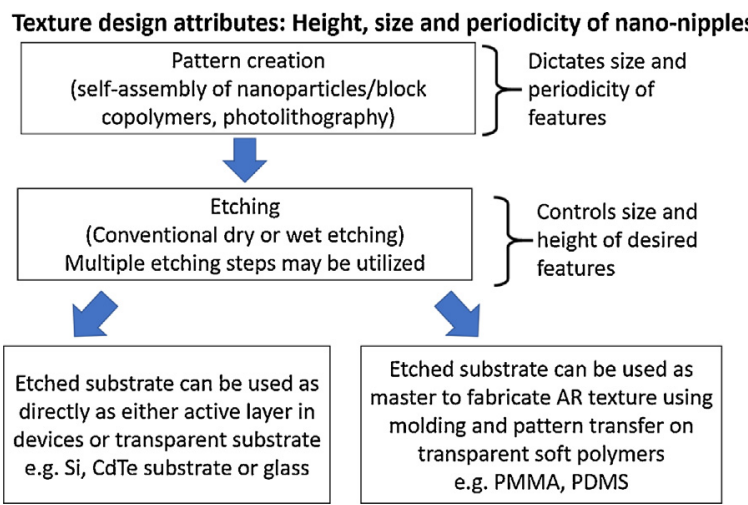

Fig. 45. Processing flow chart for manufacturing AR surface structures or coatings.

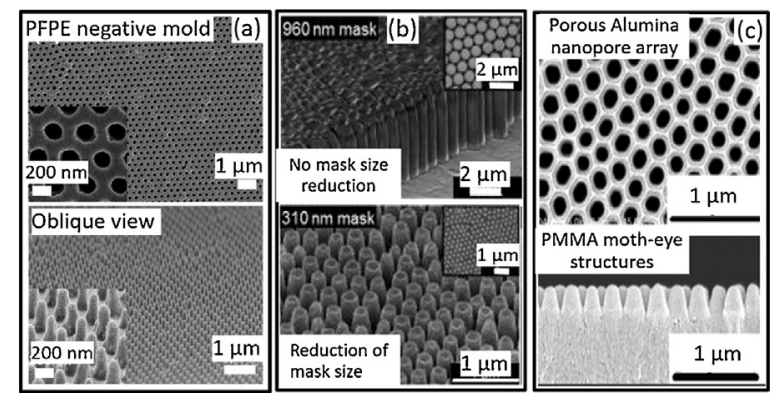

Fig. 46. (a) (top) Negative perfluoropolyether (PFPE) mold obtained by stamping on to Si-master (bottom) oblique views of moth eye structure fabricated on FTO glass using NAO 63 resin pressed onto PFPE mold [104] (reproduced with permission from John Wiley And Sons); (b) CdTe nanopillars formed by plasma etching using $\mathrm{SiO}_{2}$ nanoparticles as etching masks with (bottom) and without (top) mask size reduction [52] (reprinted with permission. Copyright 2017 American Vacuum Society); (c) top view of porous nanopores in alumina fabricated using anodizing and wet etching and oblique view of imprinted PMMA plate [175] (reproduced with permission from John Wiley And Sons).
A moth-eye pattern fabricated on Si wafers can be used as a master template to create a SWS on optically transparent polymeric materials and resins (Fig. 46(a)) by imprinting and pattern transfer techniques. Si-masters with moth-eye architectures can also be fabricated onto Si wafers using conventional photolithography [148] or laser interference lithography [174] and etching. Similarly, a team has created PDMS stamps by casting PDMS onto self-assembled PS spheres that can be used for nanoimprint lithography and patterning [165].

Self-assembled block copolymer (BCP) domains can also be used as templates to fabricate AR structures. The domain size and distribution can be easily controlled by changing the molecular weights of individual polymer blocks [222]. BCP domains are then selectively infused with Au nanoparticles [42,71], iron oxide [202] or alumina [222] to create metallic masks for subsequent etching.

In another approach, porous alumina templates were used to fabricate moth eye structures [59,175,261,293]. Aluminum substrate was first anodically oxidized to obtain porous alumina followed by multiple etching cycles using phosphoric acid solutions to widen the pores [261]. The size, shape and distribution of pores can be controlled by controlling the anodizing and etching times. These porous alumina molds can then be used to transfer the moth eye pattern onto soft polymeric materials by imprinting. Fig. 46(c) shows PMMA plates imprinted from a porous alumina mold with feature size $200 \mathrm{~nm}$ height and period [175]. The alumina mold can also be used as a patterning roll in a roll-to-roll imprinting on transparent polymers such as PET for large scale production $[59,261]$.

\subsubsection{Anti-reflection functional surfaces and product applications}

AR coatings have been studied for solar cell applications to enhance the sunlight collection due to enhanced transmittance and thus increase the efficiency of solar cells [53]. Increased power conversion efficiencies using AR structures have been reported in a variety of solar cell structures including organic solar cells [57,174], crystalline $\mathrm{Si}$ solar cells [222], moth eye textured $\mathrm{TiO}_{2} /$ perovskite solar cells [148], dye sensitized solar cells (DSSC) [104] and triple junction InGaP/InGaAs/Ge solar cells [287]. Furthermore, some of the AR coatings, owing to their surface roughness, also show a selfcleaning effect, which is beneficial for keeping the surface of solar cells clean $[104,174]$. AR structures fabricated using the techniques mentioned above are either directly incorporated into the device architecture or applied on the transparent electrode or protective encapsulation.

A liquid silica solgel-based coating fabricated from a PDMS stamp using a single step nanoimprinting process was reported. This method is suitable for large area applications such as solar panels and is scalable to a roll-to-roll process [262]. A 2.8\% increase in power output from a c-Si solar cell module was achieved using the above AR coating. This coating was also successfully used as an anti-glare film for smartphone screens resulting in improved readability.

AR coatings also have wide range of potential applications in displays and photography to reduce glare. A team of researchers fabricated PMMA plates with moth eye structures using alumina template and reported their potential use (9\% increase in transmittance) in optical diffusers to enhance the extraction efficiency of LED light [175]. Similarly, moth eye structures were imprinted from perfluoropolyether (PFPE) molds onto $\mathrm{ZnO}$ or PEDOT:PSS buffer layers in Organic Light Emitting Device (OLED) structures as well as on the back side of Indium Tin Oxide (ITO) electrode, showing an enhancement in the external quantum efficiency (EQE) of the OLEDs [299]. AR coatings have also been used in fiber optics laser systems to reduce damage due to highenergy lasers. High transmittance reaching the theoretical maximum limit was achieved using moth eye structures fabricated on one side of a Nd:YAG single crystal [46]. Another study reported less than $0.2 \%$ total optical loss in the $500 \mathrm{~nm}-2500 \mathrm{~nm}$ range using AR structures formed on fused silica substrates for potential applications for high power laser systems [71]. 
SWS coatings and films can also be employed in readers/display applications to reduce glare and improve readability. A flexible polyethylene terephthalate (PET)/triacetyl cellulose (TAC) AR film was reported which can be used for flexible display applications [253]. Moth-eye films are also incorporated in several commercial display products such as TFT touchscreen displays developed by AndersDX [122] as well as in Samsung's ultra-black technology TV screens [121]. A plasma etching process developed by the Fraunhofer Institute for Applied Optics and Precision Engineering (AR-plas ${ }^{\mathbb{R}}$ ) was used to create AR lenses by Jenoptik [123].

\section{Summary and future directions}

In conclusion, surface textures including their design architectures, materials and functionalities offer an important mechanism as a tool for realizing the next generation of functional products across multiple industrial sectors. The diverse textures discovered in biological organisms offer a well-established platform for engineering functional surfaces using novel materials and natural strategies involving combination of features- shapes, sizes, hierarchical organization length scales, aspect ratios, isotropy/anisotropy, region selectivity and many more of basic building blocks (features). These textures are manufacturable in production factories in 1-D, 2-D and 3-D features on polymers, metals, semiconductors, ceramics and composites using established industrial production processes to achieve reliability, reproducibility and repeatability in the manufacturing of millions of consumer products. As discussed above, measurement techniques and procedures are essential to analyze complex textures from nature as well as for quality control in production. Table 2 summarizes the natural examples, relevant texture components, the manufacturing processes and product applications discussed above.

The field of bio-inspired texturing, and in general, the field of bio-inspired systems is at the nascent stage. Today, the field is driven largely by curiosity rather than "production-by-design."
There is a vast directory of textures in nature. Selection of suitable application specific texture(s) need not only scientific but critical engineering considerations, such as understanding degradation modes of textures, when making decisions for what texture to select and how to select. Further, existing materials are limited in their ability to regenerate (self-healing) allowing adaptive control when reacting to their operating environments. Nature has mastered this talent of synthesizing self-healing material surfaces, regenerative material surfaces and sub-surfaces, sustainable biodegradability, and more. Nature demonstrates extraordinary ability to effectively converge various technologies including micro and submicron technologies, top-down and bottom-up processing like 3-D printing and etching, massive data driven adaptive and distributed intelligence and more for realizing multifunctional surfaces and systems specific to the species' natural habitat as a result of continuous combat and survival, over a long period of time. Though, unlike nature, synthetic engineering is limited in the use of redundancy and forgiveness when allowing high fault tolerance and reliability. The future of bio-inspired science and engineering is assembling in the direction of nature/biology- driven designs, materials, processes and systems as an onset of a new era of "convergence" or "biolization" or also called "biogicalization". The goal will be to enhance human performance and oneness with the ecosystem.

\section{Acknowledgements}

Authors sincerely acknowledge CIRP scholars for inviting multiyear Cross Scientific Technical Committee (STC) Working Group (WG) on bio-inspired surface texturing for advanced manufacturing and enriching discussions. APM and SB acknowledge partial support from the National Science Foundation (NSF) Grant No. OIA-1457888; and APM acknowledges partial support from the 21st Century Endowed Chair Professorship fund for supporting this work.

Table 2

Highlights of key examples of biological texture components, functions and manufacturing of bio-inspired products.

\begin{tabular}{|c|c|c|c|c|c|}
\hline \multirow[t]{2}{*}{ Examples in nature } & \multirow[t]{2}{*}{ Functionality } & \multicolumn{2}{|c|}{ Texture design attributes } & \multirow[t]{2}{*}{ Manufacturing processes } & \multirow[t]{2}{*}{ Product applications } \\
\hline & & Common & Differentiating & & \\
\hline $\begin{array}{l}\text { Hairy attachment } \\
\text { pads on the feet } \\
\text { of gecko, flies, } \\
\text { beetle, spiders }\end{array}$ & Dry adhesion & $\begin{array}{l}\text { Size and } \\
\text { distribution of } \\
\text { features }\end{array}$ & $\begin{array}{l}\text { Aspect ratio, } \\
\text { hierarchy, } \\
\text { orientation }\end{array}$ & $\begin{array}{l}\text { Bottom up: chemical vapor deposition } \\
\text { (CVD) } \\
\text { Sequential: top down manufacturing of } \\
\text { mold followed by casting/imprinting }\end{array}$ & $\begin{array}{l}\text { Medical adhesives, reusable adhesive } \\
\text { tapes, robotic pads, space grippers }\end{array}$ \\
\hline $\begin{array}{l}\text { Lotus leaf, rice leaf, } \\
\text { rose petals. } \\
\text { Water strider } \\
\text { legs, butterfly } \\
\text { wings }\end{array}$ & $\begin{array}{l}\text { Super- } \\
\text { hydrophobicity }\end{array}$ & $\begin{array}{l}\text { Size and } \\
\text { distribution of } \\
\text { features }\end{array}$ & $\begin{array}{l}\text { Orientation, } \\
\text { hierarchy }\end{array}$ & $\begin{array}{l}\text { Top down: lithography/etching, laser } \\
\text { surface texturing } \\
\text { Bottom up: colloidal self-assembly and } \\
\text { coating techniques, CVD } \\
\text { Sequential: top down process followed } \\
\text { by imprinting or bottom up growth }\end{array}$ & $\begin{array}{l}\text { Self-cleaning paints, water resistant } \\
\text { coatings/fabrics, glass, surgical } \\
\text { instruments, anti-corrosive coatings }\end{array}$ \\
\hline $\begin{array}{l}\text { Desert beetle, } \\
\text { Salvinia molesta }\end{array}$ & $\begin{array}{l}\text { Controlled } \\
\text { wettability }\end{array}$ & $\begin{array}{l}\text { Size and } \\
\text { distribution of } \\
\text { features }\end{array}$ & $\begin{array}{l}\text { Periodic variation } \\
\text { of surface } \\
\text { chemistry }\end{array}$ & $\begin{array}{l}\text { Top down: laser lithography, laser } \\
\text { surface treatment } \\
\text { Sequential: top down manufacturing of } \\
\text { mold followed by hot pulling }\end{array}$ & Fog/water harvesting \\
\hline $\begin{array}{l}\text { Butterfly wings, } \\
\text { peacock feathers, } \\
\text { Mollusk shells, } \\
\text { opal }\end{array}$ & $\begin{array}{l}\text { Structural } \\
\text { coloration }\end{array}$ & $\begin{array}{l}\text { Size and } \\
\text { distribution of } \\
\text { features }\end{array}$ & Periodicity & $\begin{array}{l}\text { Top down: Lithography/etching, } \\
\text { anodization, } \\
\text { Bottom up: colloidal self-assembly and } \\
\text { coating techniques }\end{array}$ & $\begin{array}{l}\text { Displays, pigment-free colors for } \\
\text { paints, textiles and makeup, security, } \\
\text { color filters }\end{array}$ \\
\hline $\begin{array}{l}\text { Moth eyes, cicada } \\
\text { wings, lycaenid } \\
\text { butterfly }\end{array}$ & Anti-reflectivity & $\begin{array}{l}\text { Size and } \\
\text { distribution of } \\
\text { features }\end{array}$ & $\begin{array}{l}\text { Shape, aspect ratio } \\
\text { (height) }\end{array}$ & $\begin{array}{l}\text { Top down: Lithography/etching } \\
\text { Sequential: Self-assembly followed by } \\
\text { etching }\end{array}$ & $\begin{array}{l}\text { Solar cell packaging, displays, laser } \\
\text { guides, photographic and optical lenses }\end{array}$ \\
\hline $\begin{array}{l}\text { Snake/fish scales, } \\
\text { Salvinia molesta, } \\
\text { tree frog. cricket } \\
\text { leg attachment } \\
\text { pads }\end{array}$ & $\begin{array}{l}\text { Drag and friction } \\
\text { management }\end{array}$ & $\begin{array}{l}\text { Size and } \\
\text { distribution of } \\
\text { features }\end{array}$ & Orientation & $\begin{array}{l}\text { Top down: Laser surface texturing } \\
\text { Bottom up: Direct bio-replication, } \\
\text { Sequential: Top-down patterning } \\
\text { followed by imprinting/casting }\end{array}$ & $\begin{array}{l}\text { Drag reducing coatings/paints for } \\
\text { submarines as well as planes, } \\
\text { swimsuits, wet and dry lubrication } \\
\text { strategies for gears, valves, bio- } \\
\text { implants }\end{array}$ \\
\hline $\begin{array}{l}\text { Shark skin, mollusc } \\
\text { shells, white crab, } \\
\text { lotus and rice leaf }\end{array}$ & Anti-fouling & $\begin{array}{l}\text { Size and } \\
\text { distribution of } \\
\text { features }\end{array}$ & Shape & $\begin{array}{l}\text { Bottom up: Direct bio-replication } \\
\text { Sequential: Top-down patterning } \\
\text { followed by imprinting/casting }\end{array}$ & $\begin{array}{l}\text { Anti-fouling paints/coatings, anti- } \\
\text { bacterial coatings for medical and } \\
\text { surgical use }\end{array}$ \\
\hline
\end{tabular}




\section{References}

[1] Abdel-Aal HA (2013) On Surface Structure and Friction Regulation in Reptilian Limbless Locomotion. Journal of the Mechanical Behavior of Biomedical Materials 22:115-135.

[2] Abdel-Aal HA, El Mansori M, Mezghani S (2010) Multi-Scale Investigation of Surface Topography of Ball Python (Python regius) Shed Skin in Comparison to Human Skin. Tribology Letters 37:517-527.

[3] Abdel-Aal HA, El Mansori M (2011) Reptilian Skin as a Biomimetic Analogue for the Design of Deterministic Tribosurfaces. in Gruber P, Bruckner D, Hellmich C, Schmiedmayer H-BStachelberger H, Gebeshuber IC, (Eds.) Biomimetics - Materials, Structures and Processes: Examples, Ideas and Case Studies, Springer, Berlin Heidelberg51-79.

[4] Abdel-Aal HA, El Mansori M (2013) Tribological Analysis of the Ventral Scale Structure in a Python regius in Relation to Laser Textured Surfaces. Topography: Metrology Properties 1:15001-15018.

[5] Abdul-Rahman HS, Jiang XJ, Scott PJ (2013) Freeform Surface Filtering Using the Lifting Wavelet Transform. Precision Engineering 37:187-202.

[6] Abdulhussein AT, Kannarpady GK, Wright AB, Ghosh A, Biris AS (2016) Current Trend in Fabrication of Complex Morphologically Tunable Superhydrophobic Nano Scale Surfaces. Applied Surface Science 384:311-332.

[7] Ahmad I, Kan C (2016) A Review on Development and Applications of Bioinspired Superhydrophobic. Textiles Materials 9:892.

[8] Amabili M, Giacomello A, Meloni S, Casciola CM (2015) Unraveling the Salvinia Paradox: Design Principles for Submerged Superhydrophobicity. Advanced Materials Interfaces 2:1500248.

[9] Arzt E, Gorb S, Spolenak R (2003) From Micro to Nano Contacts in Biological Attachment Devices. Proceedings of the National Academy of Sciences 100:1060310606.

[10] Autumn K, Sitti M, Liang YA, Peattie AM, Hansen WR, Sponberg S, Kenny TW, Fearing R, Israelachvili JN, Full RJ (2002) Evidence for van der Waals Adhesion in Gecko Setae. Proceedings of the National Academy of Sciences United States of America 99:12252-12256.

[11] Babu DJ, Mail M, Barthlott W, Schneider JJ (2017) Superhydrophobic Vertically Aligned Carbon Nanotubes for Biomimetic Air Retention under Water (Salvinia Effect). Advanced Materials Interfaces 1-6. 1700273.

[12] Bai H, Wang L, Ju J, Sun R, Zheng Y, Jiang L (2014) Efficient Water Collection on Integrative Bioinspired Surfaces with Star-shaped Wettability Patterns. Advanced Materials 26:5025-5030.

[13] Bai XQ, Xie GT, Fan H, Peng ZX, Yuan CQ, Yan XP (2013) Study on Biomimetic Preparation of Shell Surface Microstructure for Ship Antifouling. Wear 306:285-295

[14] Bariani P, De Chiffre L, Hansen HN, Horsewell A (2005) Investigation on the Traceability of Three Dimensional Scanning Electron Microscope Measurements Based on the Stereo-pair Technique. Precision Engineering 29:219-228.

[15] Barthlott W, Schimmel T, Wiersch S, Koch K, Brede M, Barczewski M, Walheim S, Weis A, Kaltenmaier A, Leder A, Bohn HF (2010) The Salvinia Paradox: Superhydrophobic Surfaces with Hydrophilic Pins for Air Retention under Water. Advanced Materials 22:2325-2328.

[16] Bartl MH, Galusha JW, Richey LR, Gardner JS, Cha JN (2008) Discovery of a Diamond-based Photonic Crystal Structure in Beetle Scales. Physical Review EStatistical Nonlinear and Soft Matter Physics 77:2-5.

[17] Baum MJ, Heepe L, Fadeeva E, Gorb SN (2014) Dry Friction of Microstructured Polymer Surfaces Inspired by Snake Skin. Beilstein Journal of Nanotechnology 5:1091-1103.

[18] Baum MJ, Heepe L, Gorb SN (2014) Friction Behavior of a Microstructured Polymer Surface Inspired by Snake Skin. Beilstein Journal of Nanotechnology 5:83-97.

[19] Baum MJ, Kovalev AE, Michels J, Gorb SN (2014) Anisotropic Friction of the Ventral Scales in the Snake Lampropeltis Getula Californiae. Tribology Letters 54:139-150.

[20] Bechert DW, Bruse M, Hage W (2000) Experiments with Three-dimensional Riblets as an Idealized Model of Shark Skin. Experiments in Fluids 28:403-412.

[21] Behrens BA, Krimm R, Jocker J, Reithmeier E, Roth B, Rahlves M (2013) Method to Emboss Holograms into the Surface of Sheet Metals. Key Engineering Materials 549:125-132

[22] Bernhard CG, Gemne G, Sällström J (1970) Comparative Ultrastructure of Corneal Surface Topography in Insects with Aspects on Phylogenesis and Function. Zeitschrift Für Vergleichende Physiologie 67:1-25.

[23] Bernhard CG, Miller WH (1962) A Corneal Nipple Pattern in Insect Compound Eyes. Acta Physiologica Scandinavica 56:385-386.

[24] Bers AV, Diaz ER, da Gama BAP, Vieira-Siglva F, Dobretsov S, Valdivia N, Thiel M, Scardino AJ, McQuaid CD, Sudgen HE, Thomason JC, Wahl M (2010) Relevance of Mytilid Shell Microtopographies for Fouling Defence - A Global Comparison. Biofouling 26:367-377.

[25] Bhushan B (2012) Bioinspired Structured Surfaces. Langmuir 28:1698-1714.

[26] Bhushan B (2009) Biomimetics: Lessons from Nature-An Overview. Philosophical Transactions of the Royal Society A: Mathematical Physical and Engineering Sciences 367:1445-1486.

[27] Bhushan B (2016) Nanofabrication Techniques Used for Lotus-like Structures. Biomimetics. Biological and Medical Physics, Biomedical Engineering, Springe Cham85-96.

[28] Bhushan B, Nosonovsky M (2010) The Rose Petal Effect and the Modes of Superhydrophobicity. Philosophical Transactions of the Royal Society A: Mathematical Physical and Engineering Sciences 368:4713-4728.

[29] Bhushan B, Sayer RA (2006) Gecko Feet: Natural Attachment Systems for Smart Adhesion-mechanism Modeling, and Development of Bio-inspired Materials. Applied Scanning Probe Methods X, Springer, Berlin Heidelberg1-61.

[30] BIPM IEC, IFCC I, IUPAC I, ISO O (2012) The international vocabulary of metrology: basic and general concepts and associated terms (VIM). JCGM 200: 2012. 3rd edn. JCGM (Joint Committee for Guides in Metrology).
[31] Bixler GD, Bhushan B (2012) Biofouling: Lessons from Nature. Philosophical Transactions of the Royal Society 370:2381-2417.

[32] Bixler GD, Bhushan B (2013) Fluid Drag Reduction and Efficient Self-cleaning with Rice Leaf and Butterfly Wing Bioinspired Surfaces. Nanoscale 5:7685.

[33] Bixler GD, Bhushan B (2015) Rice and Butterfly Wing Effect Inspired Low Drag and Antifouling Surfaces: A Review. Critical Reviews in Solid State and Materials Sciences 40:1-37.

[34] Bixler GD, Bhushan B (2013) Bioinspired Micro/Nanostructured Surfaces for Oil Drag Reduction in Closed Channel Flow. Soft Matter 9:1620-1635.

[35] Bixler GD, Bhushan B (2012) Bioinspired Rice Leaf and Butterfly Wing Surface Structures Combining Shark Skin and Lotus Effects. Soft Matter 8:11271.

[36] Bixler GD, Theiss A, Bhushan B, Lee SC (2014) Anti-fouling Properties of Microstructured Surfaces Bio-inspired by Rice Leaves and Butterfly Wings. Journal of Colloid and Interface Science 419:114-133.

[37] Blau PJ (2012) Use of Textured Surfaces to Mitigate Sliding Friction and Wear of Lubricated and Non-Lubricated Contacts, Oak Ridge National Laboratory, TN United States. http://dx.doi.org/10.2172/1039238 (accessed 13.05.18)https:// info.ornl.gov/sites/publications/files/Pub34466.pdf.

[38] Boyde A (1973) Quantitative Photogrammetric Analysis and Qualitative Stereoscopic analysis of SEM Images. Journal of Microscopy 98:452-471.

[39] Boyle BM, French TA, Pearson RM, McCarthy BG, Miyake GM (2017) Structural Color for Additive Manufacturing: 3D-Printed Photonic Crystals from Block Copolymers. ACS Nano 11:3052-3058.

[40] Bradshaw E, Rudall PJ, Devey DS, Thomas MM, Glover BJ, Bateman RM (2010) Comparative Labellum Micromorphology of the Sexually Deceptive Temperate Orchid Genus Ophrys: Diverse Epidermal Cell Types and Multiple Origins of Structural Colour. Botanical Journal of the Linnean Society 162:504-540.

[41] Breur R (2013) Non-toxic, Anti-fouling Coating for Marine Vessels. Advanced Coatings \& Surface Technology 26:7-9.

[42] Brunner R, Deparnay A, Helgert M, Burkhardt M, Lohmüller T, Spatz JP (2008) Product piracy from nature: biomimetic microstructures and interfaces for high-performance optics In K. Creath (Ed.), Proc. of SPIE vol. 7057:705705.

[43] Brunner R, Sandfuchs O, Pacholski C, Morhard C, Spatz J (2012) Lessons from Nature: Biomimetic Subwavelength Structures for High-performance Optics. Laser \& Photonics Reviews 6:641-659.

[44] Bruzzone AAG, Costa HL, Lonardo PM, Lucca DA (2008) Advances in Engineered Surfaces for Functional performance. CIRP Annals - Manufacturing Technology 57:750-769.

[45] Burrows M, Sutton G (2013) Interacting Gears Synchronize Propulsive Leg Movements in a Jumping Insect. Science 341:1254-1256.

[46] Busse LE, Frantz JA, Shaw LB, Aggarwal ID, Sanghera JS (2015) Review of Antireflective Surface Structures on Laser Optics and Windows. Applied Optics 54:F303.

[47] Cai J, Qi L (2015) Recent Advances in Antireflective Surfaces Based on Nanostructure Arrays. Materials Horizons 2:37-53.

[48] Carmignato S (2014) Calibration. CIRP Encyclopedia of Production Engineering, Springer, Berlin Heidelberg1-5.

[49] Carneiro K, Jensen CP, Jørgensen JF, Garnœs J, McKeown PA (1995) Roughness Parameters of Surfaces by Atomic Force Microscopy. CIRP Annals 44:517-522.

[50] Cassie ABD, Baxter S (1944) Wettability of Porous Surfaces. Transactions of the Faraday Society 40:546.

[51] Cerman Z, Striffler BF, Barthlott W (2009) Dry in the Water: The Superhydrophobic Water Fern Salvinia - A Model for Biomimetic Surfaces. Functional Surfaces in Biology, Springer, Netherlands97-111.

[52] Chan L, Ghoshal A, DeCuir Jr EA, Chen YP, Morse DE, Gordon MJ (2017) Fabrication and Optical Behavior of Graded-index, Moth-eye Antireflective Structures in CdTe. Journal of Vacuum Science E Technology B Nanotechnology and Microelectronics: Materials Processing Measurement and Phenomena 35:11201.

[53] Chandra A, Anderson G, Melkote S, Gao W, Haitjema H, Wegener K (2014) Role of Surfaces and Interfaces in Solar Cell Manufacturing. CIRP Annals 63:797-819.

[54] Chen B, Zhong G, Goldberg Oppenheimer P, Zhang C, Tornatzky H, Esconjauregui S, Hofmann S, Robertson J (2015) Influence of Packing Density and Surface Roughness of Vertically-aligned Carbon Nanotubes on Adhesive Properties of Gecko-inspired Mimetics. ACS Applied Materials and Interfaces 7:3626-3632.

[55] Chen H, Zhang X, Ma L, Che D, Zhang D, Sudarshan TS (2014) Investigation on Large-area Fabrication of Vivid Shark Skin with Superior Surface Functions. Applied Surface Science 316:124-131.

[56] Chen Z, Zhao W, Xu J, Mo M, Peng S, Zeng Z, Wu X, Xue Q (2015) Designing Environmentally Benign Modified Silica Resin Coatings with Biomimetic Textures for Antibiofouling. RSC Advances 5:36874-36881.

[57] De Chen J, Zhou L, Ou QD, Li YQ, Shen S, Lee ST, Tang JX (2014) Enhanced Light Harvesting in Organic Solar Cells Featuring a Biomimetic Active Layer and Self-cleaning Antireflective Coating. Advanced Energy Materials 4:1-8.

[58] Cheng F, Gao J, Luk TS, Yang X (2015) Structural Color Printing Based on Plasmonic Metasurfaces of Perfect Light Absorption. Scientific Reports 5:11045.

[59] Chuang C, Lu D, Wang P, Lee W (2016) Fabrication of Antireflective Polymer Films with Subwavelength Structures Using Roll to Roll Nanoimprint Lithography. IEEE 2016 Symposium on Design Test Integration and Packaging of MEMS/ MOEMS (DTIP) 1-5.

[60] Cuenat A (2014) Scanning Probe and Particle Beam Microscopy. Fundamental Principles of Engineering Nanometrology, Elsevier: 205-239.

[61] Cuervo P, López DA, Cano JP, Sánchez JC, Rudas S, Estupiñán H, Toro A (2016) Development of Low Friction Snake-inspired Deterministic Textured Surfaces. Surface Topography: Metrology and Properties 4:1-17.

[62] Cui Y, Ju Y, Xu B, Wang P, Kojima N, Ichioka K (2014) Mimicking a Gecko's Foot with Strong Adhesive Strength Based on a Spinnable Vertically Aligned Carbon Nanotube Array. RSC Advances 4:9056-9060.

[63] Cui Y, Wang B, Zhang M (2013) Optimizing Reaction Condition for Synthesizing Spinnable Carbon Nanotube Arrays by Chemical Vapor Deposition. Journal of Materials Science 48:7749-7756. 
[64] De Chiffre L, Kunzmann H, Peggs GN, Lucca DA (2003) Surfaces in Precision Engineering, Microengineering and Nanotechnology. CIRP Annals Manufacturing Technology 52:561-577.

[65] De Chiffre L, Lonardo P, Trumpold H, Lucca DA, Goch G, Brown CA, Raja J, Hansen HN (2000) Quantitative Characterisation of Surface Texture. CIRP Annals - Manufacturing Technology 49:635-652.

[66] Deiwick A, Fadeeva E, Koch L, Gebauer R, Chichkov B, Schlie-Wolter S, Elena Fadeeva AD (2014) Functional Titanium Lotus-Topography Promotes the Osteoinduction of Human Adipose-Derived Stem Cells In Vitro. Journal of Nanomedicine \&' Nanotechnology 5:1.

[67] Denkena B, Köhler J, Krawczyk T (2014) Grinding of Riblet Structures on Free Formed Compressor Blades. Advanced Materials Research 907:463-473.

[68] Denkena B, Köhler J, Wang B (2010) Manufacturing of Functional Riblet Structures by Profile Grinding. CIRP Journal of Manufacturing Science and Technology 3:14-26.

[69] Dewan R, Marinkovic M, Noriega R, Phadke S, Salleo A, Knipp D (2009) Light Trapping in Thin-film Silicon Solar Cells with Submicron Surface Texture. Optics Express 17:23058.

[70] Dey S, Dkhar B (1992) An Unusual Type of Corneal Nipple in the Earwig, Forficula sp., with a Possible Anti-reflection Role. Micron and Microscopica Acta 23:337-339.

[71] Diao Z, Dirks J-H, Spatz J (2016) Bio-inspired, Nanostructured Anti-reflective Surfaces for Laser Applications. Conference on Lasers and Electro-Optics, OSA. ATh3 K.5.

[72] Do YS, Park JH, Hwang BY, Lee S-M, Ju B-K, Choi KC (2013) Plasmonic Color Filter and its Fabrication for Large-Area Applications. Advanced Optical Materials $1: 133-138$.

[73] Du S, Liu C, Huang D (2015) A Shearlet-based Separation Method of 3D Engineering Surface Using High Definition Metrology. Precision Engineering 40:55-73.

[74] Eisenhaure J, Kim S (2017) A Review of the State of Dry Adhesives: Biomimetic Structures and the Alternative Designs They Inspire. Micromachines 8:1-38.

[75] Erdemir A (2005) Review of Engineered Tribological Interfaces for Improved Boundary Lubrication. Tribology International 38:249-256.

[76] Estler WT (2016) Traceability. CIRP Encyclopedia of Production Engineering, Springer, Berlin Heidelberg1-4

[77] Evans CJ, Bryan JB (1999) "Structured", “Textured" or "Engineered" Surfaces. CIRP Annals - Manufacturing Technology 48:541-556.

[78] Favret EA, Fuentes NO (2009) Functional Properties of Biological Surfaces. Functional Properties of Bio-Inspired Surfaces, World Scientific: 1.

[79] Feng L, Li S, Li Y, Li H, Zhang L, Zhai J, Song Y, Liu B, Jiang L, Zhu D (2002) Superhydrophobic Surfaces: From Natural to Artificial. Advanced Materials 14:18571860https://onlinelibrary.wiley.com/doi/abs/10.1002/adma.200290020.

[80] Feng L, Zhang Y, Xi J, Zhu Y, Wang N, Xia F, Jiang L (2008) Petal Effect: A Superhydrophobic State with High Adhesive Force. Langmuir 24:4114-4119.

[81] Filippov AE, Gorb SN (2016) Modelling of the Frictional Behaviour of the Snake Skin Covered by Anisotropic Surface Nanostructures. Scientific Reports 6:23539.

[82] Frankiewicz C, Attinger D (2016) Texture and Wettability of Metallic Lotus Leaves. Nanoscale 8:3982-3990.

[83] Frost SJ, Mawad D, Higgins MJ, Ruprai H, Kuchel R, Tilley RD, Myers S, Hook JM, Lauto A (2016) Gecko-inspired Chitosan Adhesive for Tissue Repair. NPG Asia Materials $8 \mathrm{e} 280$

[84] Fu F, Chen Z, Zhao Z, Wang H, Shang L, Gu Z, Zhao Y (2017) Bio-inspired Selfhealing Structural Color Hydrogel. Proceedings of the National Academy of Sciences 114:5900-5905.

[85] Fu Y, Tippets CA, Donev EU, Lopez R (2016) Structural Colors: From Natural to Artificial Systems Wiley Interdisciplinary Reviews. Nanomedicine and Nanobiotechnology 8:758-775.

[86] Fudouzi H (2011) Tunable Structural Color in Organisms and Photonic Materials for Design of Bioinspired Materials. Science and Technology of Advanced Materials 12:64704

[87] Gao W, Rigout M, Owens H (2016) Self-assembly of Silica Colloidal Crystal Thin Films with Tuneable Structural Colours over a Wide Visible Spectrum. Applied Surface Science 380:12-15.

[88] Gao W, Rigout M, Owens H (2017) Optical Properties of Cotton and Nylon Fabrics Coated with Silica Photonic Crystals. Optical Materials Express 7:341353.

[89] Gao W, Rigout M, Owens H (2017) The Structural Coloration of Textile Materials Using Self-assembled Silica Nanoparticles. Journal of Nanoparticle Research 19:303.

[90] Gonzalez FL, Gordon MJ (2014) Bio-inspired, Sub-wavelength Surface Structures for Ultra-broadband Omni-directional Anti-reflection in the Mid and Far IR. Optics Express 22:12808.

[91] Gorb SN, (Ed.) (2009), Functional Surfaces in Biology, Springer, Netherlands.

[92] Gorb SN (2007) Visualisation of Native Surfaces by Two-step Molding. Microscopy Today 15:44-46.

[93] Greiner C, Schäfer M (2015) Bio-inspired Scale-like Surface Textures and Their Tribological Properties. Bioinspiration \& Biomimetics 10:44001.

[94] Guittard F, Darmanin T (2017) Bioinspired Superhydrophobic Surfaces: Advances and Applications with Metallic and Inorganic Materials, Pan Stanford Pub..

[95] Guo C, Tian Q, Wang H, Sun J, Du L, Wang M, Zhao D (2017) Roller Embossing Process for the Replication of Shark-skin-inspired Micro-riblets. Micro \& Nano Letters 12:439-444.

[96] Guo Z, Liu W (2007) Biomimic from the Superhydrophobic Plant Leaves in Nature: Binary Structure and Unitary Structure. Plant Science 172:1103-1112.

[97] Guo Z, Liu W, Su BL (2011) Superhydrophobic Surfaces: From Natural to Biomimetic to Functional. Journal of Colloid and Interface Science 353:335-355.

[98] Haitjema H (2014) Measurement Uncertainty. CIRP Encyclopedia of Production Engineering, Springer, Berlin Heidelberg852-857.
[99] Hansen HN, Carneiro K, Haitjema H, De Chiffre L (2006) Dimensional Micro and Nano Metrology. CIRP Annals - Manufacturing Technology 55:721-743.

[100] Hansen WR, Autumn K (2005) Evidence for Self-cleaning in Gecko Setae. Proceedings of the National Academy of Sciences 102:385-389.

[101] Hansma P, Elings V, Marti O, Bracker C (1988) Scanning Tunneling Microscopy and Atomic Force Microscopy: Application to Biology and Technology. Science 242:209-216.

[102] Hawkes EW, Christensen DL, Han AK, Jiang H, Cutkosky MR (2015) Grasping without Squeezing: Shear Adhesion Gripper with Fibrillar Thin Film. 2015 IEEE International Conference on Robotics and Automation (ICRA) 2305--2312.

[103] Hazel J, Stone M, Grace MS, Tsukruk VV (1999) Nanoscale Design of Snake Skin for Reptation Locomotions via Friction Anisotropy. Journal of Biomechanics 32:477-484.

[104] Heo SY, Koh JK, Kang G, Ahn SH, Chi WS, Kim K, Kim JH (2014) Bifunctional Moth-eye Nanopatterned Dye-sensitized Solar Cells: Light-harvesting and Self-cleaning Effects. Advanced Energy Materials 4:1-7.

[105] Ho AYY, Luong Van E, Lim CT, Natarajan S, Elmouelhi N, Low HY, Vyakarnam M (2014) Lotus Bioinspired Superhydrophobic, Self-cleaning Surfaces from Hierarchically Assembled Templates. Journal of Polymer Science Part B: Polymer Physics 52:603-609.

[106] Hocken RJ, Chakraborty N, Brown C (2005) Optical Metrology of Surfaces. CIRP Annals - Manufacturing Technology 54:169-183.

[107] Holmberg K, Andersson P, Erdemir A (2012) Global Energy Consumption Due to Friction in Passenger Cars. Tribology International 47:221-234.

[108] Holmberg K, Andersson P, Nylund N-O, Mäkelä K, Erdemir A (2014) Global Energy Consumption Due to Friction in Trucks and Buses. Tribology International 78:94-114.

[109] http://arldesignsllc.com/superhydrophobic-surfaces/. (Retrieved 10 October 2017).

[110] http://lotusleafcoatings.com/coatings/\#hydrofoe. (Retrieved 6 October 2017).

[111] http://nanogriptech.com/. (Retrieved 9 October 2017).

[112] http://nbdnano.com/. (Retrieved 5 October 2017).

[113] http://stethoskin.com/about-stethoskin/. (Retrieved 10 October 2017).

[114] http://www.loreal.com/research-and-innovation/ push-back-the-boundaries-of-knowledge/the-secrets-of-color. (Retrieved 5 October 2017).

[115] http://www.micanti.com/. (Retrieved 10 October 2017).

[116] http://www.morphotonix.com/. (Retrieved 5 October 2017).

[117] http://www.nanosecurity.ca/. (Retrieved 5 October 2017).

[118] http://www.speedousa.com/fastskin. (Retrieved 8 October 2017).

[119] https://geckskin.umass.edu/. (Retrieved 8 October 2017).

[120] https://greenshieldfinish.com/. (Retrieved 10 October 2017)

[121] https://news.samsung.com/global/ultra-black-technology-for-those-of-uswho-dont-turn-off-the-lights-to-watch-tv. (Retrieved 5 October 2017).

[122] https://www.andersdx.com/blog/sunlight-readable-displays, https://www. andersdx.com/blog/sunlight-readable-displays. (Retrieved 10 October 2017).

[123] https://www.jenoptik.com/products/optical-systems/ solutions-and-integrated-technologies/optical-coatings ar-plas-plasma-etching-coating. (Retrieved 10 October 2017).

[124] https://www.lufthansa-technik.com/famos. (Retrieved 8 October 2017).

[125] https://www.sharklet.com/. (Retrieved 10 October 2017).

[126] https://www.tresclean.eu/. (Retrieved 10 October 2017).

[127] Hu DL, Nirody J, Scott T, Shelley MJ (2009) The Mechanics of Slithering Locomotion. Proceedings of the National Academy of Sciences of the United States of America 106:10081-10085.

[128] Hu H, Tian H, Shao J, Wang Y, Li X, Tian Y, Ding Y, Lu B (2017) Friction Contribution to Bioinspired Mushroom-Shaped Dry Adhesives. Advanced Materials Interfaces 1700016.

[129] Hu S, Xia Z, Dai L (2013) Advanced Gecko-foot-mimetic Dry Adhesives Based on Carbon Nanotubes. Nanoscale 5:475-486.

[130] Huang W, Wang X (2013) Biomimetic Design of Elastomer Surface Pattern for Friction Control under Wet Conditions. Bioinspiration \&' Biomimetics 8:4600146006.

[131] Hunt J, Bhushan B (2011) Nanoscale Biomimetics Studies of Salvinia molesta for Micropattern Fabrication. Journal of Colloid and Interface Science 363:187192

[132] Hwang I, Yi H, Choi J, Jeong HE (2017) Fabrication of Bioinspired Dry Adhesives by CNC Machining and Replica Molding. International Journal of Precision Engineering and Manufacturing 18:1239-1244.

[133] Ingram A, Parker A (2008) A Review of the Diversity and Evolution of Photonic Structures in Butterflies, Incorporating the Work of John Huxley (The Natural History Museum, London from 1961 to 1990). Philosophical Transactions of the Royal Society B: Biological Sciences 363:2465-2480.

[134] ISO 25178:2 (2012) Geometrical Product Specifications (GPS) - Surface Texture: Areal - Part 2: Terms, Definitions and Surface Texture Parameters, International Organization for Standardization.

[135] ISO 25178:3 (2012) Geometrical Product Specifications (GPS) - Surface Texture: Areal - Part 3: Specification Operators, International Organization for Standardization.

[136] ISO 4287 (1997) Geometrical Product Specifications (GPS) - Surface Texture: Profile Method - Terms, Definitions and Surface Texture Parameters, International Organization for StandardizationRetrieved from, https://www.iso.org/ standard/10132.html.

[137] ISO 4288 (1996) Geometrical Product Specifications (GPS) - Surface Texture: Profile Method - Rules and Procedures for the Assessment of Surface Texture, International Organization for Standardization.

[138] Jagota A, Hui C-Y (2011) Adhesion, Friction, and Compliance of Bio-mimetic and Bio-inspired Structured Interfaces. Materials Science and Engineering: R: Reports 72:253-292. 
[139] Ji C, Lee K-T, Xu T, Zhou J, Park HJ, Guo LJ (2017) Engineering Light at the Nanoscale: Structural Color Filters and Broadband Perfect Absorbers. Advanced Optical Materials 1700368.

[140] Ji S, Park J, Lim H (2012) Improved Antireflection Properties of Moth Eye Mimicking Nanopillars on Transparent Glass: Flat Antireflection and Color Tuning. Nanoscale 4:4603.

[141] Ji S, Song K, Nguyen TB, Kim N, Lim H (2013) Optimal Moth Eye Nanostructure Array on Transparent Glass Towards Broadband Antireflection. ACS Applied Materials \& Interfaces 5:10731-10737.

[142] Jiang H, Hawkes EW, Fuller C, Estrada MA, Suresh SA, Abcouwer N, Han AK, Wang S, Ploch CJ, Parness A, Cutkosky MR (2017) A Robotic Device Using Gecko-inspired Adhesives Can Grasp and Manipulate Large Objects in Microgravity. Science Robotics 2:1-11.

[143] Jiang T, Guo Z, Liu W (2015) Biomimetic Superoleophobic Surfaces: Focusing on Their Fabrication and Applications. Journal of Materials Chemistry A 3:1811-1827.

[144] Jiang W, Lei B, Liu H, Niu D, Zhao T, Chen B, Yin L, Shi Y, Liu X (2016) Fabrication of Directional Nanopillars with High-aspect-ratio Using a Stretching Imprint Process with a Microcavity Mold. Nanoscale 9:2172-2177.

[145] Jiang XJ, Whitehouse DJ (2012) Technological Shifts in Surface Metrology CIRP Annals - Manufacturing Technology 61:815-836.

[146] Jin K, Cremaldi JC, Erickson JS, Tian Y, Israelachvili JN, Pesika NS (2014) Biomimetic Bidirectional Switchable Adhesive Inspired by the Gecko. Advanced Functional Materials 24:574-579.

[147] Johnson KL, Kendall K, Roberts AD (1971) Surface Energy and the Contact of Elastic Solids. Proceedings of the Royal Society A: Mathematical Physical and Engineering Sciences 324:301-313.

[148] Kang SM, Jang S, Lee JK, Yoon J, Yoo DE, Lee JW, Choi M, Park NG (2016) MothEye $\mathrm{TiO}_{2}$ Layer for Improving Light Harvesting Efficiency in Perovskite Solar Cells. Small 12:2443-2449.

[149] Kasem H, Tsipenyuk A, Varenberg M (2015) Biomimetic Wall-shaped Hierarchical Microstructure for Gecko-like Attachment. Soft Matter 11:2909-2915.

[150] Kavalenka MN, Vüllers F, Lischker S, Zeiger C, Hopf A, Röhrig M, Rapp BE Worgull M, Hölscher H (2015) Bioinspired Air-Retaining Nanofur for Drag Reduction. ACS Applied Materials and Interfaces 7:10651-10655.

[151] Bin Khaled W, Sameoto D (2014) Fabrication and Characterization of Thermoplastic Elastomer Dry Adhesives with High Strength and Low Contamination. ACS Applied Materials and Interfaces 6:6806-6815.

[152] Bin Khaled W, Sameoto D (2013) Anisotropic Dry Adhesive via Cap Defects. Bioinspiration \& Biomimetics 8:44002.

[153] Khedir KR, Kannarpady GK, Ryerson C, Biris AS (2017) An Outlook on Tunable Superhydrophobic Nanostructural Surfaces and Their Possible Impact on Ice Mitigation. Progress in Organic Coatings 112:304-318.

[154] Kim GH, An T, Lim G (2017) Bioinspired Structural Colors Fabricated with ZnO Quasi-Ordered Nanostructures. ACS Applied Materials and Interfaces 9:1905719062.

[155] Kim T, Park J, Sohn J, Cho D (2016) Bioinspired, Highly Stretchable, and Conductive Dry Adhesives Based on 1D-2D Hybrid Carbon Nanocomposites for All-in-One ECG Electrodes. ACS Nano 10:4770-4778.

[156] Kim T, Shin R, Jung M, Lee J, Park C, Kang S (2016) Drag Reduction Using Metallic Engineered Surfaces with Highly Ordered Hierarchical Topographies: Nanostructures on Micro-riblets. Applied Surface Science 367:147-152.

[157] Kinoshita S, Yoshioka S (2005) Structural Colors in Nature: The Role of Regularity and Irregularity in the Structure. ChemPhysChem 6:1443-1459.

[158] Kizilkan E, Strueben J, Staubitz A, Gorb SN (2017) Bioinspired Photocontrollable Microstructured Transport Device. Science Robotics 2. eaak9454.

[159] Ko D-H, Tumbleston JR, Henderson KJ, Euliss LE, DeSimone JM, Lopez R, Samulski ET (2011) Biomimetic Microlens Array with Antireflective "motheye" Surface. Soft Matter 7:6404.

[160] Koch K, Barthlott W (2009) Superhydrophobic and Superhydrophilic Plant Surfaces: an Inspiration for Biomimetic Materials. Philosophical Transactions of the Royal Society A: Mathematical Physical and Engineering Sciences 367:1487-1509.

[161] Koch K, Bhushan B, Barthlott W (2010) Multifunctional Plant Surfaces and Smart Materials. Springer Handbook of Nanotechnology, Springer, Berlin Heidelberg1399-1436.

[162] Koch K, Bhushan B, Barthlott W (2009) Multifunctional Surface Structures of Plants: an Inspiration for Biomimetics. Progress in Materials Science 54:137178.

[163] Koch K, Bohn HF, Barthlott W (2009) Hierarchically Sculptured Plant Surfaces and Superhydrophobicity. Langmuir 25:14116-14120.

[164] Kondo Y, Koyama T, Sasaki S (2013) Tribological Properties of Ionic Liquids. Ionic Liquids - New Aspects for the Future, INTECH Open Access Publisher.

[165] Kuo W-K, Hsu J-J, Nien C-K, Yu HH (2016) Moth-Eye-Inspired Biophotonic Surfaces with Antireflective and Hydrophobic Characteristics. ACS Applied Materials E Interfaces 8:32021-32030.

[166] Lang AW, Motta P, Hidalgo P, Westcott M (2008) Bristled Shark Skin: A Microgeometry for Boundary Layer Control? Bioinspiration \& Biomimetics 3:46005.

[167] Latthe S, Terashima C, Nakata K, Fujishima A (2014) Superhydrophobic Surfaces Developed by Mimicking Hierarchical Surface Morphology of Lotus Leaf. Molecules 19:4256-4283.

[168] Leach R (2014) Surface Texture. CIRP Encyclopedia of Production Engineering, Springer, Berlin Heidelberg1-4.

[169] Leach R (2014) Fundamental Principles of Engineering Nanometrology, 2nd ed. Elsevier.

[170] Leach R, Haitjema H (2010) Bandwidth Characteristics and Comparisons of Surface Texture Measuring Instruments. Measurement Science and Technology 21:32001.

[171] Leach RK, Giusca CL, Haitjema H, Evans C, Jiang X (2015) Calibration and Verification of Areal Surface Texture Measuring Instruments. CIRP Annals Manufacturing Technology 64:797-813.
[172] Lee KT, Jang JY, Park SJ, Ji C, Yang SM, Guo LJ, Park HJ (2016) Angle-Insensitive and CMOS-Compatible Subwavelength Color Printing. Advanced Optical Materials 4:1696-1702.

[173] Lee M, (Ed.) (2014), Remarkable Natural Material Surfaces and Their Engineering Potential, Springer International Publishing.

[174] Leem JW, Kim S, Lee SH, Rogers JA, Kim E, Yu JS (2014) Efficiency Enhancement of Organic Solar Cells Using Hydrophobic Antireflective Inverted Moth-eye Nanopatterned PDMS Films. Advanced Energy Materials 4:1-7.

[175] Li J, Zhu J, Gao X (2014) Bio-inspired High-performance Antireflection and Antifogging Polymer Films. Small 10:2578-2582.

[176] Li M, Huang W, Wang X (2015) Bioinspired, Peg-studded Hexagonal Patterns for Wetting and Friction. Biointerphases 10:31008

[177] Li Q (2017) A Practical Fabrication Method of the Gecko-inspired Easyremoval Skin Adhesives. Biosurface and Biotribology 3:66-74.

[178] Li Y, Zhang H, Yao Y, Li T, Zhang Y, Li Q, Dai Z (2015) Transfer of Vertically Aligned Carbon Nanotube Arrays onto Flexible Substrates for Gecko-inspired Dry Adhesive Application. RSC Advances 5:46749-46759.

[179] Li Y, Zhang J, Yang B (2010) Antireflective Surfaces Based on Biomimetic Nanopillared Arrays. Nano Today 5:117-127.

[180] Liu K, Jiang L (2011) Bio-inspired Design of Multiscale Structures for Function Integration. Nano Today 6:155-175.

[181] Liu K, Jiang L (2012) Bio-Inspired Self-Cleaning Surfaces. Annual Review of Materials Research 42:231-263.

[182] Liu K, Yao X, Jiang L (2010) Recent Developments in Bio-inspired Special Wettability. Chemical Society Reviews 39:3240.

[183] Liu Y, Li S, Niu S, Cao X, Han Z, Ren L (2016) Bio-inspired Micro-nano Structured Surface with Structural Color and Anisotropic Wettability on Cu Substrate. Applied Surface Science 379:230-237.

[184] Liu Y, Shigley J, Hurwit K (1999) Iridescent Color of a Shell of the Mollusk Pinctada margaritifera Caused by Diffraction. Optics Express 4:177-182.

[185] Liu Z, Yin W, Tao D, Tian Y (2015) A Glimpse of Superb Tribological Designs in Nature. Biotribology 122:11-23.

[186] Lonardo PM, Lucca DA, De Chiffre L (2002) Emerging Trends in Surface Metrology. CIRP Annals - Manufacturing Technology 51:701-723.

[187] Long J, Fan P, Gong D, Jiang D, Zhang H, Li L, Zhong M (2015) Superhydrophobic Surfaces Fabricated by Femtosecond Laser with Tunable Water Adhesion: From Lotus Leaf to Rose Petal. ACS Applied Materials E Interfaces 7:98589865.

[188] Long J, Fan P, Zhong M, Zhang H, Xie Y, Lin C (2014) Superhydrophobic and Colorful Copper Surfaces Fabricated by Picosecond Laser Induced Periodic Nanostructures. Applied Surface Science 311:461-467.

[189] Lou S, Guo X, Fan T, Zhang D (2012) Butterflies: Inspiration for Solar Cells and Sunlight Water-splitting Catalysts. Energy E' Environmental Science 5:9195.

[190] Luo Y, Liu Y, Anderson J, Li X, Li Y (2015) Improvement of Water-repellent and Hydrodynamic Drag Reduction Properties on Bio-inspired Surface and Exploring Sharkskin Effect Mechanism. Applied Physics A 120:369-377.

[191] Luo Y, Xu X, Li D, Song W (2016) Recent Developments in Fabricating Drag Reduction Surfaces Covering Biological Sharkskin Morphology. Reviews in Chemical Engineering 32:93-113.

[192] Luo Y, Yuan L, Li J, Wang J (2015) Boundary Layer Drag Reduction Research Hypotheses Derived from Bio-inspired Surface and Recent Advanced Applications. Micron 79:59-73.

[193] Ma FM, Li W, Liu AH, Yu ZL, Ruan M, Feng W, Chen HX, Chen Y (2017) Geometrical Effect, Optimal Design and Controlled Fabrication of Bio-inspired Micro/nanotextures for Superhydrophobic Surfaces. Materials Research Express 4:92001.

[194] Ma S, Wang D, Liang Y, Sun B, Gorb SN, Zhou F (2015) Gecko-inspired but Chemically Switched Friction and Adhesion on Nanofibrillar Surfaces. Small 11:1131-1137.

[195] Magin CM, Cooper SP, Brennan AB (2010) Non-toxic Antifouling Strategies. Materials Today 13:36-44.

[196] Mahdavi A, Ferreira L, Sundback C, Nichol JW, Chan EP, Carter DJD, Bettinger C, Patanavanich S, Chignozha L, Ben-Joseph E, Galakatos A, Pryor H, Pomerantseva I, Masiakos PT, Faquin W, Zumbuehl A, Hong S, Borenstein J, Vacanti J, Langer R, Karp JM (2008) A Biodegradable and Biocompatible Gecko-inspired Tissue Adhesive. Proceedings of the National Academy of Sciences of the United States of America 105:2307-2312, http://www.pnas.org/content/105/7/2307. short.

[197] Malshe A, Rajurkar K, Samant A, Hansen HN, Bapat S, Jiang W (2013) Bioinspired Functional Surfaces for Advanced Applications. CIRP Annals Manufacturing Technology 62:607-628.

[198] Maréchal J-P, Hellio C (2009) Challenges for the Development of New NonToxic Antifouling Solutions. International Journal of Molecular Sciences 10:4623-4637.

[199] Marvi H, Hu DL (2012) Friction Enhancement in Concertina Locomotion of Snakes. Journal of The Royal Society Interface 9:3067-3080.

[200] Marvi H, Song S, Sitti M (2015) Experimental Investigation of Optimal Adhesion of Mushroomlike Elastomer Microfibrillar Adhesives. Langmuir 31:10119-10124.

[201] Micciché M, Arzt E, Kroner E (2014) Single Macroscopic Pillars as Model System for Bioinspired Adhesives: Influence of Tip Dimension, Aspect Ratio and Tilt Angle. ACS Applied Materials E Interfaces 6:7076-7083.

[202] Mokarian-Tabari P, Senthamaraikannan R, Glynn C, Collins TW, Cummins C Nugent D, O’Dwyer C, Morris MA (2017) Large Block Copolymer Self-Assembly for Fabrication of Subwavelength Nanostructures for Applications in Optics. Nano Letters 17:2973-2978.

[203] Mondal B, Mac Giolla Eain M, Xu QF, Egan VM, Punch J, Lyons AM (2015) Design and Fabrication of a Hybrid Superhydrophobic-Hydrophilic Surface That Exhibits Stable Dropwise Condensation. ACS Applied Materials and Interfaces 7:23575-23588. 
[204] Morikawa J, Ryu M, Seniutinas G, Badytis A, Maximova K, Wang X, Zamengo M, Ivanova EP, Juodkazis S (2016) Nanostructured Antireflective and Thermoisolative Cicada Wings. Langmuir 32:4698-4703.

[205] Müller F, Kunz C, Gräf S (2016) Bio-Inspired Functional Surfaces Based on Laser-Induced Periodic Surface Structures. Materials 9:476.

[206] Nam H, Song K, Ha D, Kim T (2016) Inkjet Printing Based Mono-layered Photonic Crystal Patterning for Anti-counterfeiting Structural Colors. Scientific Reports 6:30885.

[207] De Nicola F, Castrucci P, Scarselli M, Nanni F, Cacciotti I, De Crescenzi M (2015) Super-hydrophobic Multi-walled Carbon Nanotube Coatings for Stainless Steel. Nanotechnology 26:145701.

[208] Nir S, Reches M (2016) Bio-inspired Antifouling Approaches: The Quest towards Non-toxic and Non-biocidal Materials. Current Opinion in Biotechnology 39:48-55.

[209] Nishimoto S, Bhushan B (2012) Bioinspired Self-cleaning Surfaces with Superhydrophobicity, Superoleophobicity and Superhydrophilicity. RSC Advances 3:671-690.

[210] Niu S, Li B, Mu Z, Yang M, Zhang J, Han Z, Ren L (2015) Excellent Structurebased Multifunction of Morpho Butterfly Wings: A Review. Journal of Bionic Engineering 12:170-189.

[211] Nørgaard T, Dacke M (2010) Fog-basking Behaviour and Water Collection Efficiency in Namib Desert Darkling beetles. Frontiers in Zoology 7:23.

[212] Pagani L, Qi Q Jiang X, Scott PJ (2017) Towards a New Definition of Areal Surface Texture Parameters on Freeform Surface. Measurement 109:281-291.

[213] Park C-S, Shrestha VR, Yue W, Gao S, Lee S-S, Kim E-S, Choi D-Y (2017) Structural Color Filters Enabled by a Dielectric Metasurface Incorporating Hydrogenated Amorphous Silicon Nanodisks. Scientific Reports 7:2556.

[214] Park YW, Kim TW (2016) Fabrication of a Micro-riblet Shark Skin-like Surface using a WEDM Process. Journal of the Korean Society of Tribologists and Lubrication Engineers 32:201-206.

[215] Parker AR, Hegedus Z, Watts RA (1998) Solar-absorber Antireflector on the Eye of an Eocene Fly (45 Ma). Proceedings of the Royal Society B: Biological Sciences 265:811--815.

[216] Parker AR (2000) 515 Million Years of Structural Colour. Journal of Optics A: Pure and Applied Optics 2:R15-R28.

[217] Parker AR, Lawrence CR (2001) Water Capture by a Desert Beetle. Nature 414:33-34.

[218] Parness A, Hilgendorf T, Daniel P, Frost M, White V, Kennedy B (2013) Controllable ON-OFF Adhesion for Earth Orbit Grappling Applications. 2013 IEEE Aerospace Conference 1--11.

[219] Peters J, Bryan JB, Estler WT, Evans C, Kunzmann H, Lucca DA, Sartori S, Sato H, Thwaite EG, Vanherck P, Hocken RJ, Peklenik J, Pfeifer T, Trumpold H, Vorburger TV (2001) Contribution of CIRP to the Development of Metrology and Surface Quality Evaluation During the Last Fifty Years. CIRP Annals Manufacturing Technology 50:471-488

[220] Pu X, Li G, Huang H (2016) Preparation, Anti-biofouling and Drag-reduction Properties of a Biomimetic Shark Skin Surface. Biology Open 5:389-396.

[221] Rahlves M, Kelb C, Rezem M, Schlangen S, Boroz K, Gödeke D, Ihme M, Roth B (2015) Digital Mirror Devices and Liquid Crystal Displays in Maskless Lithography for Fabrication of Polymer-based Holographic Structures. Journal of Micro/Nanolithography MEMS and MOEMS 14:41302.

[222] Rahman A, Ashraf A, Xin H, Tong X, Sutter P, Eisaman MD, Black CT (2015) Sub-50-nm Self-assembled Nanotextures for Enhanced Broadband Antireflection in Silicon Solar Cells. Nature Communications 6:5963.

[223] Rai-Choudhury P (1997) Handbook of Microlithography, Micromachining, and Microfabrication. Volume 1: Microlithography, SPIE Press.

[224] Raut HK, Ganesh VA, Nair AS, Ramakrishna S (2011) Anti-reflective Coatings: A Critical In-depth Review. Energy \&' Environmental Science 4:3779.

[225] Röhrig M, Mail M, Schneider M, Louvin H, Hopf A, Schimmel T, Worgull M, Hölscher H (2014) Nanofur for Biomimetic Applications. Advanced Materials Interfaces 1:1300083.

[226] Sahay R, Baji A, Parveen H, Ranganath AS (2017) Dry-adhesives Based on Hierarchical Poly(Methyl Methacrylate) Electrospun Fibers. Applied Physics A: Materials Science and Processing 123:1-7.

[227] Sahay R, Parveen H, Baji A, Ganesh V (2017) Fabrication of PVDF Hierarchical Fibrillar Structures Using Electrospinning for Dry-adhesive Applications. Journal of Materials 52:2435-2441.

[228] Sahoo BN, Balasubramanian K, Sucheendran M (2015) Thermally Triggered Transition of Superhydrophobic Characteristics of Micro- and Nanotextured Multiscale Rough Surfaces. Journal of Physical Chemistry C 119:14201-14213.

[229] Saito A (2011) Material Design and Structural Color Inspired by Biomimetic Approach. Science and Technology of Advanced Materials 12:64709.

[230] Santo L, Davim JP (2014) Surface Engineering Techniques and Applications. in Santo L, Davim JP, (Eds.) Surface Engineering Techniques and Applications: Research Advancements, IGI Global.

[231] Scardino AJ, Guenther J, de Nys R (2008) Attachment Point Theory Revisited: the Fouling Response to a Microtextured Matrix. Biofouling 24:45-53.

[232] Scardino AJ, Hudleston D, Peng Z, Paul NA, de Nys R (2009) Biomimetic Characterisation of Key Surface Parameters for the Development of Fouling Resistant Materials. Biofouling 25:83-93.

[233] Scardino AJ, de Nys R (2011) Mini Review: Biomimetic Models and Bioinspired Surfaces for Fouling Control. Biofouling 27:73-86.

[234] Scardino AJ, Harvey E, De Nys R (2006) Testing Attachment Point Theory: Diatom Attachment on Microtextured Polyimide Biomimics. Biofouling 22:55-60.

[235] Schumacher JF, Aldred N, Callow ME, Finlay JA, Callow JA, Clare AS, Brennan AB (2007) Species-specific Engineered Antifouling Topographies: Correlations Between the Settlement of Algal Zoospores and Barnacle Cyprids. Biofouling 23:307-317.

[236] Schumacher JF, Carman ML, Estes TG, Feinberg AW, Wilson LH, Callow ME, Callow JA, Finlay JA, Brennan AB (2007) Engineered Antifouling Microtopo- graphies - Effect of Feature Size, Geometry and Roughness on Settlement of Zoospores of the Green Alga Ulva. Biofouling 23:55-62.

[237] Seago AE, Brady P, Vigneron J-P, Schultz TD (2009) Gold Bugs and Beyond: a Review of Iridescence and Structural Colour Mechanisms in Beetles (Coleoptera). Journal of The Royal Society Interface 6:S165-S184

[238] Sekiguchi Y, Sato C (2017) Experimental Investigation of the Effect of Tip Shape in Gecko-inspired Adhesive Devices under Asymmetric Detachment. Applied Adhesion Science 5:8.

[239] Shen Y, Liu S, Zhu C, Tao J, Wang G (2017) Facile Fabrication of Hierarchical Structured Superhydrophobic Surface and Its Ultra Dynamic Water Repellency. Chemical Engineering Journal 313:47-55.

[240] Shin S, Seo J, Han H, Kang S, Kim H, Lee T (2016) Bio-Inspired Extreme Wetting Surfaces for Biomedical Applications. Materials 9:116.

[241] Si G, Zhao Y, Lv J, Lu M, Wang F, Liu H, Xiang N, Huang TJ, Danner AJ, Teng J, Liu YJ (2013) Reflective Plasmonic Color Filters Based on Lithographically Patterned Silver Nanorod Arrays. Nanoscale 5:6243.

[242] Siddique RH, Faisal A, Hünig R, Bartels C, Wacker I, Lemmer U, Hölscher H (2014) Utilizing Laser Interference Lithography to Fabricate Hierarchical Optical Active Nanostructures Inspired by the Blue Morpho Butterfly. Spie 9187:91870E.

[243] Siddique RH, Gomard G, Hölscher H (2015) The Role of Random Nanostructures for the Omnidirectional Anti-reflection Properties of the Glasswing Butterfly. Nature Communications 6:6909.

[244] Simpson JT, Hunter SR, Aytug T (2015) Superhydrophobic Materials and Coatings: a Review. Reports on Progress in Physics 78:86501.

[245] Song S, Sitti M (2014) Soft Grippers Using Micro-fibrillar Adhesives for Transfer Printing. Advanced Materials 26:4901-4906.

[246] Spolenak R, Gorb S, Arzt E (2005) Adhesion Design Maps for Bio-inspired Attachment Systems. Acta Biomaterialia 1:5-13.

[247] Stavenga D, Foletti S, Palasantzas G, Arikawa K (2006) Light on the Moth-eye Corneal Nipple Array of Butterflies. Proceedings of the Royal Society B: Biological Sciences 273:661-667.

[248] Sto-sea.com (2016) StoColor Lotusan. Retrieved from http://www.sto-sea. com/en/products/product-guide/product_4814.html.

[249] Sun CH, Jiang P, Jiang B (2008) Broadband Moth-eye Antireflection Coatings on Silicon. Applied Physics Letters 92:2006-2009.

[250] Sun J, Bhushan B, Tong J (2013) Structural Coloration in Nature. RSC Advances 3:14862.

[251] Sun L, Hu X, Wu Q, Wang L, Zhao J, Yang S, Tai R, Fecht H-J, Zhang D-X, Wang LQ Jiang J-Z (2016) High Throughput Fabrication of Large-area Plasmonic Color Filters by Soft-X-ray Interference Lithography. Optics Express 24:19112.

[252] Sun M, Liang A, Zheng Y, Watson GS, Watson JA (2011) A Study of the Antireflection Efficiency of Natural Nano-arrays of Varying Sizes. Bioinspiration $\mathcal{E}^{\prime}$ Biomimetics 6:26003.

[253] Tan G, Lee J-H, Lan Y-H, Wei M-K, Peng L-H, Cheng I-C, Wu S-T (2017) Broadband Antireflection Film with Moth-eye-like Structure for Flexible Display Applications. Optica 4:678.

[254] Tao D, Gao X, Lu H, Liu Z, Li Y, Tong H, Pesika N, Meng Y, Tian Y (2017) Controllable Anisotropic Dry Adhesion in Vacuum: Gecko Inspired Wedged Surface Fabricated with Ultraprecision Diamond Cutting. Advanced Functional Materials 27:1-9.

[255] Tillmann W, Stangier D, Lopes-Dias NF, Biermann D, Krebs E (2017) Adjustment of Friction by Duplex-treated Bionic Structures for Sheet-Bulk Metal Forming. Tribology International 111:9-17.

[256] Tosello G, Haitjema H, Leach RK, Quagliotti D, Gasparin S, Hansen HN (2016) An International Comparison of Surface Texture Parameters Quantification on Polymer Artefacts Using Optical Instruments. CIRP Annals 65:529-532.

[257] Townsend A, Pagani L, Blunt L, Scott PJ, Jiang X (2017) Factors Affecting the Accuracy of Areal Surface Texture Data Extraction from X-ray CT. CIRP Annals - Manufacturing Technology 66:547-550.

[258] Tricinci O, Terencio T, Mazzolai B, Pugno NM, Greco F, Mattoli V (2015) 3D Micropatterned Surface Inspired by Salvinia molesta via Direct Laser Lithography. ACS Applied Materials and Interfaces 7:25560-25567.

[259] Tsipenyuk A, Varenberg M (2014) Use of Biomimetic Hexagonal Surface Texture in Friction Against Lubricated Skin. Journal of The Royal Society Interface 11:20140113.

[260] Umh HN, Yu S, Kim YH, Lee SY, Yi J (2016) Tuning the Structural Color of a 2D Photonic Crystal Using a Bowl-like Nanostructure. ACS Applied Materials and Interfaces 8:15802-15808.

[261] UOZU Y (2012) Continuous Roll Imprinting of Moth Eye Antireflection Surface Using Anodic Porous Alumina. Design for Innovative Value Towards a Sustainable Society, Springer, Netherlands915-917.

[262] van de Groep J, Spinelli P, Polman A (2015) Single-Step Soft-Imprinted LargeArea Nanopatterned Antireflection Coating. Nano Letters 15:4223-4228.

[263] Varenberg M, Gorb SN (2009) Hexagonal Surface Micropattern for Dry and Wet Friction. Advanced Materials 21:483-486.

[264] Vukusic P, Sambles JR (2003) Photonic Structures in Biology. Nature 424:852855.

[265] Wang H, Zhang KQ (2013) Photonic Crystal Structures with Tunable Structure Color as Colorimetric Sensors. Sensors (Basel Switzerland) 13:4192-4213.

[266] Wang M, Liu Q, Zhang H, Wang C, Wang L, Xiang B, Fan Y, Guo CF, Ruan S (2017) Laser Direct Writing of Tree-Shaped Hierarchical Cones on a Superhydrophobic Film for High-Efficiency Water Collection. ACS Applied Materials and Interfaces 9:29248-29254.

[267] Wang Y, Hu H, Shao J, Ding Y (2014) Fabrication of Well-defined Mushroomshaped Structures for Biomimetic Dry Adhesive by Conventional Photolithography and Molding. ACS Applied Materials and Interfaces 6:2213-2218.

[268] Wang Y, Shao J, Ding Y, Li X, Tian H, Hu H (2015) Effects of contact cap dimension on dry adhesion of bioinspired mushroom-shaped surfaces In Lakhtakia A, Knez M, Martín-Palma RJ (Eds.), Proc. of SPIE vol. 9429:94291E. 
[269] Wang Y, Tian H, Shao J, Sameoto D, Li X, Wang L, Hu H, Ding Y, Lu B (2016) Switchable Dry Adhesion with Step-like Micropillars and Controllable Interfacial Contact. ACS Applied Materials and Interfaces 8:10029-10037.

[270] Wang Z, Gu P, Wu X (2014) Gecko-inspired Bidirectional Double-sided Adhesives. Soft Matter 10:3301.

[271] Watson GS, Watson JA (2004) Natural Nano-structures on Insects - Possible Functions of Ordered Arrays Characterized by Atomic Force Microscopy. Applied Surface Science 235:139-144.

[272] Wen G, Guo Z, Liu W (2017) Biomimetic Polymeric Superhydrophobic Surfaces and Nanostructures: from Fabrication to Applications. Nanoscale 9:3338-3366.

[273] Wen L, Weaver JC, Lauder GV (2014) Biomimetic Shark Skin: Design Fabrication and Hydrodynamic Function. Journal of Experimental Biology 217:16561666.

[274] Wenzel RN (1936) Resistance of Solid Surfaces to Wetting by Water. American Dyestuff Reporter 25:598-601

[275] Whitney HM, Kolle M, Andrew P, Chittka L, Steiner U, Glover BJ (2009) Floral Iridescence, Produced by Diffractive Optics Acts As a Cue for Animal Pollinators. Science 323:130-133.

[276] Wilkening G, Bosse H (2005) Nano-and Micrometrology Stateof-the-Art and Future Challenges. MAPAN-Journal of Metrology Society of India 20:125-151.

[277] Wilson SJ, Hutley MC (1982) The Optical Properties of "Moth Eye" Antireflection Surfaces. Optica Acta: International Journal of Optics 29:993-1009.

[278] Wu J-H, Phillips BS, Jiang W, Sanders JH, Zabinski JS, Malshe AP (2006) Bioinspired Surface Engineering and Tribology of $\mathrm{MoS}_{2}$ Overcoated cBN-TiN Composite Coating. Wear 261:592-599.

[279] Wu S, Liu B, Su X, Zhang S (2017) Structural Color Patterns on Paper Fabricated by Inkjet Printer and Their Application in Anticounterfeiting. Journal of Physical Chemistry Letters 8:2835-2841.

[280] Xia F, Jiang L (2008) Bio-inspired, Smart, Multiscale Interfacial Materials Advanced Materials 20:2842-2858.

[281] Xie Z, Li L, Liu P, Zheng F, Guo L, Zhao Y, Jin L, Li T, Gu Z (2015) Self-assembled Coffee-ring Colloidal Crystals for Structurally Colored Contact Lenses. Small 11:926-936.

[282] Xu J, Guo Z (2013) Biomimetic Photonic Materials with Tunable Structural Colors. Journal of Colloid and Interface Science 406:1-17.

[283] Xu M, Du F, Ganguli S, Roy A, Dai L (2016) Carbon Nanotube Dry Adhesives with Temperature-enhanced Adhesion over a Large Temperature Range. Nature Communications 7:13450

[284] Xue Y, Lv P, Lin H, Duan H (2016) Underwater Superhydrophobicity: Stability, Design and Regulation, and Applications. Applied Mechanics Reviews 68:30803.

[285] Yi H, Hwang I, Lee JH, Lee D, Lim H, Tahk D, Sung M, Bae W-G, Choi S-J, Kwak MK, Jeong HE (2014) Continuous and Scalable Fabrication of Bioinspired Dry
Adhesives via a Roll-to-Roll Process with Modulated Ultraviolet-Curable Resin. ACS Applied Materials E Interfaces 6:14590-14599.

[286] Yi H, Kang M, Kwak MK, Jeong HE (2016) Simple and Reliable Fabrication of Bioinspired Mushroom-Shaped Micropillars with Precisely Controlled Tip Geometries. ACS Applied Materials and Interfaces 8:22671-22678.

[287] Yu P, Chiu M-Y, Chang C-H, Hong C-Y, Tsai Y-L, Han H-V, Wu Y-R (2014) Towards High-efficiency Multi-junction Solar Cells with Biologically Inspired Nanosurfaces. Progress in Photovoltaics: Research and Applications 22:300307.

[288] Yu S, Guo Z, Liu W (2015) Biomimetic Transparent and Superhydrophobic Coatings: From Nature and Beyond Nature. Chemical Communications 51:1775-1794.

[289] Yuan W, Zhou N, Shi L Zhang KO (2015) Structural Coloration of Colloida Fiber by Photonic Band Gap and Resonant Mie Scattering. ACS Applied Materials and Interfaces 7:14064-14071.

[290] Yuehao L, Deyuan Z (2013) Investigation on Fabricating Continuous Vivid Sharkskin Surface by Bio-replicated Rolling Method. Applied Surface Science 282:370-375.

[291] Zeng Q, Ding C, Li Q, Yuan W, Peng Y, Hu J, Zhang K-Q (2017) Rapid Fabrication of Robust, Washable, Self-healing Superhydrophobic Fabrics with Non-iridescent Structural Color by Facile Spray Coating. RSC Advances 7:8443-8452.

[292] Zhang E, Liu Y, Yu J. Lv T, Li L (2015) Fabrication of Hierarchical Gecko-inspired Microarrays Using a Three-dimensional Porous Nickel Oxide Template. Journal of Materials Chemistry B 3:6571-6575.

[293] Zhang J, Shen S, Dong XX, Chen LS (2014) Low-cost Fabrication of Large Area Sub-wavelength Anti-reflective Structures on Polymer Film Using a Soft PUA Mold. Optics Express 22:1842-1851.

[294] Zhang P, Liu S, Lv H (2013) Fabrication and Properties of Dual-Level Hierarchical Structures Mimicking Gecko Foot Hairs. Journal of Nanoscience and Nanotechnology 13:781-786.

[295] Zhang S, Chen Y (2015) Nanofabrication and Coloration Study of Artificial Morpho Butterfly Wings with Aligned Lamellae Layers. Scientific Reports 5:16637.

[296] Zhang S, Chen Y, Lu B, Liu J, Shao J, Xu C (2016) Lithographically-generated 3D Lamella Layers and Their Structural Color. Nanoscale 8:9118-9127.

[297] Zhang S, Huang J, Chen Z, Lai Y (2017) Bioinspired Special Wettability Surfaces: From Fundamental Research to Water Harvesting Applications. Small 13:1-28

[298] Zhao Y, Xie Z, Gu H, Zhu C, Gu Z (2012) Bio-inspired Variable Structural Color Materials. Chemical Society Reviews 41:3297.

[299] Zhou L, Ou Q-D, Chen J-D, Shen S, Tang J-X, Li Y, Lee S-T (2014) Light Manipulation for Organic Optoelectronics Using Bio-inspired Moth's Eye Nanostructures. Scientific Reports 4:4040. 